\title{
Simple Adaptive Control for Spacecraft Trajectory Tracking Under Uncertainties and Perturbations
}

\author{
by \\ David Rogers \\ A thesis submitted to the Faculty of Graduate and Postdoctoral Affairs \\ in partial fulfillment of the requirements for the degree of \\ Master of Applied Science \\ in \\ Aerospace Engineering \\ Carleton University \\ Ottawa, Ontario \\ (C) 2018 \\ David Rogers
}




\section{Abstract}

Orbital debris is a growing problem that poses a collision risk to spacecraft and is expected to worsen exponentially according to Kessler syndrome as each collision between two bodies fragments them into three or more. Active removal of debris by a chaser spacecraft is necessary to prevent runaway growth in the number of objects but removal is complicated by the presence of perturbation forces and uncertainties about debris parameters such as mass. Capturing and towing debris and performing similar proximity operations will require a control strategy that can perform trajectory tracking that is robust to these uncertainties.

This thesis presents the use of Simple Adaptive Control (SAC), a direct adaptive control method, to perform trajectory tracking in the presence of mass and perturbation uncertainties. A new laboratory facility was developed for this work which included an air bearing testbed and robotic platforms representing spacecraft. The author contributed significantly to the development of this facility, notably creating the software environment that enabled simulations and experiments.

The main experiment in this work involves a chaser platform performing a circular inspection trajectory around a target, representing an important phase in debris removal or other proximity operations. Four different scenarios were tested with different conditions: nominal conditions which involved no perturbations or mass uncertainties, increasing the mass by $50 \%$ without informing the system or retuning control gains, subjecting the system to perturbation forces, and finally combining the mass change with perturbations for the worst case scenario. The trajectory tracking performance of SAC and PD control were compared in all four scenarios. Both simulation and experimental results agree that SAC is a viable control strategy for this purpose that significantly outperforms a PD controller in all the conditions considered, notably providing more consistent performance across several trials in the presence of randomly fluctuating perturbations. It was also discovered that perturbations had a more significant impact on trajectory tracking performance than mass changes in this context, though the combined effect had the largest impact. 


\section{Acknowledgements}

I sincerely thank my supervisor, Dr. Steve Ulrich. His continuous support, clear direction and friendly attitude make him the best supervisor anybody could hope to have during graduate studies. I would also like to thank my family, my colleague Kirk Hovell and Dr. Ronald Miller, Professor and Chair of Mechanical and Aerospace Engineering at Carleton University. 


\section{Table of Contents}

Abstract $\quad$ ii

Acknowledgements $\quad$ iii

List of Tables $\quad$ vi

List of Figures $\quad$ vii

Chapter 1 Introduction 1

1.1 Motivation ........................ 1

1.2 Problem Statement . . . . . . . . . . . . . . . . . . . 1

1.3 Previous Work . . . . . . . . . . . . . . . . . 2

1.4 Thesis Objectives . . . . . . . . . . . . . . . . . . 3

1.5 Contributions ......................... 4

1.6 Organization ........................ 4

$\begin{array}{lll}\text { Chapter } 2 & \text { Relative Motion Dynamics } & 6\end{array}$

2.1 Relative Orbit Geometry . . . . . . . . . . . . . . 6

2.2 Nonlinear Relative Dynamics . . . . . . . . . . . . . . . 7

2.3 Linearized Relative Dynamics . . . . . . . . . . . . . . . . . . 14

2.4 Double Integrator Model . . . . . . . . . . . . . . . . . . . . . 14

2.5 State Space Representation . . . . . . . . . . . . . . 16

$\begin{array}{lll}\text { Chapter } 3 & \text { Simple Adaptive Control } & 17\end{array}$

3.1 Ideal Model . . . . . . . . . . . . . . . . . . . . 17

3.2 Control Objective . . . . . . . . . . . . . . . . . . . 18

3.3 Control Law and Adaptation Mechanisms . . . . . . . . . . . . 18

3.4 Ideal Control and State Trajectory . . . . . . . . . . . . . 21

3.5 Tracking under Non-ideal Conditions . . . . . . . . . . . . . . . 22 
$\begin{array}{lll}\text { Chapter } 4 \quad \text { Simulations } & 25\end{array}$

4.1 Numerical Simulations . . . . . . . . . . . . . . . . . . 25

4.2 Attitude Control . . . . . . . . . . . . . . . . . 28

4.3 Perturbations . . . . . . . . . . . . . . . . . 28

4.4 Controller Tuning . . . . . . . . . . . . . . . . . . . . . 29

4.5 Tracking Error . . . . . . . . . . . . . . . . . . . . . . . 29

4.6 Contextualizing Tracking Error: Astrobee Free-flying Robots Inside the International Space Station . . . . . . . . . . . . . . . . . 31

4.7 Simulation Results and Discussion . . . . . . . . . . . . . . . . 32

4.8 Conclusion . . . . . . . . . . . . . . . . . . . . . . . . 44

Chapter $5 \quad$ Experiments $\quad 45$

5.1 Experimental Facility . . . . . . . . . . . . . . . . 45

5.2 Lab Contributions . . . . . . . . . . . . . . . . 47

5.3 Experimental Setup . . . . . . . . . . . . . . . . . . . . 49

5.4 Perturbations . . . . . . . . . . . . . . . . 51

5.5 Statistical Analysis . . . . . . . . . . . . . . . 54

5.6 Experimental Results and Discussion . . . . . . . . . . . . 54

5.7 Conclusion . . . . . . . . . . . . . . . . . . . 69

$\begin{array}{lll}\text { Chapter } 6 & \text { Conclusion } & 70\end{array}$

6.1 Thesis Summary . . . . . . . . . . . . . . . 70

6.2 Future Work . . . . . . . . . . . . . . . . . . 71

$\begin{array}{ll}\text { Bibliography } & 73\end{array}$ 


\section{List of Tables}

$4.1 \quad$ Initial conditions for simulations and experiments. . . . . . . 26 


\section{List of Figures}

$2.1 \quad$ Local-vertical-local-horizontal reference frame. $\ldots \ldots \ldots \ldots$

4.1 Visual representation of parameters used in simulations and experiments . . . . . . . . . . . . . . . 27

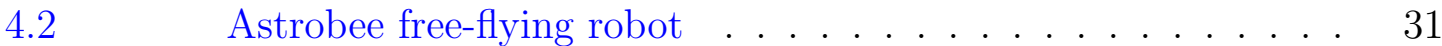

4.3 Trajectory tracking simulation under nominal conditions using (a) PD control and (b) SAC . . . . . . . . . . . . . . 33

4.4 Trajectory tracking errors in simulation under nominal conditions during (a) transient state and (b) steady state . . . . . . 34

4.5 Trajectory tracking simulation with an unknown mass added to the system using (a) PD control and (b) SAC . . . . . . 36

4.6 Trajectory tracking errors in simulation with an unknown mass added to the system . . . . . . . . . . . . . 37

4.7 Trajectory tracking simulation with perturbations using (a) PD control and (b) SAC . . . . . . . . . . . . . . . . 39

$4.8 \quad$ Trajectory tracking errors in simulation with perturbations . . 40

4.9 Trajectory tracking simulation under off-nominal conditions using (a) PD control and (b) SAC . . . . . . . . . . . . 42

4.10 Trajectory tracking errors in simulation under off-nominal conditions . . . . . . . . . . . . . . . . . . 43

$5.1 \quad$ SPOT platform built at Carleton University . . . . . . . 46

5.2 Overview of the Spacecraft Proximity Operations Testbed . . 47

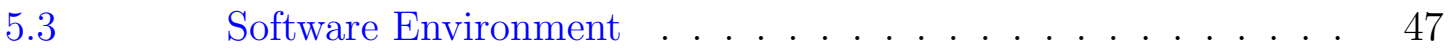

5.4 Overview of the MATLAB/Simulink diagram used in the experimental facility configured for (a) simulations and (b) experiments 48

$5.5 \quad$ Experimental setup (all dimensions in $\mathrm{mm}$ ) . . . . . . 50

$5.6 \quad$ Experiment initial conditions $\ldots \ldots \ldots \ldots \ldots \ldots$ 
5.7 Perturbation device: Honeywell HV180 fan mounted on rotating platform with laser pointer . . . . . . . . . . . . . 52

5.8 Trajectory tracking experiment under nominal conditions using (a) PD control and (b) SAC . . . . . . . . . . . . 55

5.9 Trajectory tracking errors in experiment under nominal conditions during (a) transient state and (b) steady state . . . . . 56

5.10 Trajectory tracking experiment with an unknown mass added to the system using (a) PD control and (b) SAC . . . . . . 58

5.11 Trajectory tracking errors in experiment with an unknown mass added to the system . . . . . . . . . . . . . . . 59

5.12 Trajectory tracking experiment with perturbations using (a) PD control and (b) SAC . . . . . . . . . . . . . . . 61

5.13 Trajectory tracking errors in experiment with perturbations .

5.14 Trajectory tracking experiment under off-nominal conditions using (a) PD control and (b) SAC . . . . . . . . . . . . 65

5.15 Trajectory tracking errors in experiment under off-nominal conditions . . . . . . . . . . . . . . . 66

5.16 SAC stabilizing control gain matrix $\boldsymbol{K}_{e}(t) \in \mathbb{R}^{3 \times 3}$ in experiment under off-nominal conditions . . . . . . . . . . . . . . . 68 


\section{Chapter 1}

\section{Introduction}

\subsection{Motivation}

Orbital debris is a growing problem exacerbated by an anti-satellite missile test in 2007 and a collision between two satellites in $2009^{1}$. By 2015 there were approximately 13,000 debris objects larger than $10 \mathrm{~cm}$ orbiting the Earth [1]. Collisions between debris objects or between a debris object and a satellite occur at extreme speeds that fragment colliding bodies into many smaller objects which risk further collision (analogous to a runaway nuclear reaction). Kessler discovered this exponential growth in the number of orbital debris and the effect was named Kessler Syndrome [2]. The European Space Agency estimates that approximately 5 to 10 significant debris objects must be actively removed per year to prevent runaway growth in the number of orbital debris [3]. Active removal requires an active chaser spacecraft which can rendezvous with and capture debris objects, ultimately towing them to a lower orbit where atmospheric drag will cause the debris to descend rapidly and be incinerated by frictional heating during re-entry. The technology required for such an application and spacecraft proximity operations in general is an important area of research and development and the motivation behind this work.

\subsection{Problem Statement}

One of the difficulties of space debris removal is that the chaser may have to inspect, capture and tow targets of unknown mass along trajectories while experiencing perturbations. If the target mass was known beforehand and there were no perturbations to cause the chaser to deviate from its trajectory, a simple control strategy could be implemented and tuned to the known conditions. However, when the target mass is

\footnotetext{
${ }^{1}$ EPFL Space Engineering Center, CleanSpace One (accessed October 22, 2017) https:// espace.epfl.ch/CleanSpaceOne_1
} 
unknown and there are perturbations present, trajectory tracking is no longer trivial and a simple Proportional-Integral-Derivative (PID) controller cannot guarantee adequate performance.

Instead, an adaptive control strategy that adapts to such uncertainties and perturbations may be employed. In other words, this would allow the chaser to perform trajectory tracking with unknown masses and perturbations as its control can adapt to the unknown conditions. As a result, the chaser spacecraft can inspect debris by tracking a trajectory around it and, following a successful rendezvous and capture maneuver, tow the debris to a disposal orbit to be incinerated during atmospheric reentry - all while avoiding collisions by accurately tracking the desired trajectory and being robust to uncertainties. These trajectory tracking capabilities are also useful for other proximity operations such as on-orbit servicing of the International Space Station (ISS) performed by Astrobee free-flying robots [4].

In this context, this thesis is concerned with the adaptive control required for the chaser to perform trajectory tracking under uncertainties and perturbations.

\subsection{Previous Work}

The problem of control and trajectory tracking for relative motion between spacecraft has received significant attention in previous work. This section presents previous work relevant to the topic of spacecraft control and trajectory tracking in order to situate this thesis into the context of related work that has already been completed.

Capturing target debris necessarily involves close-proximity operations. Typically, the chaser would be close enough to the target for the relative dynamics to be described by the Clohessy-Wiltshire equations [5]. The dynamics can be simplified further to a double-integrator model when the time scale is negligible compared to the orbital period of the target and the distance between the chaser and target is no more than a few meters [6]. This reduces the dynamics to a linear model which is appealing for control purposes due to the abundance of linear continuous control laws for trajectory tracking which are suggested by the relevant literature [7]. Unfortunately, in the context of the problem studied by this thesis which involves uncertain mass and perturbations, these linear control methods can perform poorly because 
they are model-based and require information about the plant (mass and inertia) as well as models for perturbations, all of which are unknown for this problem.

One solution to the problem of the unknown mass and perturbations proposed in literature is to implement indirect adaptive control methods [8]. These methods must explicitly determine the unknown parameters in real-time which are then used to determine the control gains, but this requires a good understanding of the plant dynamics model. An indirect adaptive control method was proposed by de Queiroz et al. [9] where an adaptation law uses a highly accurate dynamics model to identify the unknown mass for use in the control law. Unfortunately these real-time methods for determining unknown parameters are computationally expensive, prohibitively so for applications with smaller spacecraft that have limited on-board processors.

Another solution to the problem of the unknown mass and perturbations proposed in literature is to implement direct adaptive control methods [10]. Unlike indirect methods, direct methods a plant dynamics model or estimates of plant parameters. This technique has been recently developed and implemented successfully by Ulrich et al. [11]. Specifically, Simple Adaptive Control (SAC) theory [10] was used to develop the direct adaptive control law. This controller was tested both in simulation and in experiment using the Synchronized Position Hold Engage Reorient Experimental Satellites (SPHERES) air bearing testbed at the Massachusetts Institute of Technology's Space Systems Laboratory. However, the single-run experiment was highly limited since only a short straight line relative trajectory was considered. In addition, this recent experimental work did not investigate the effects of unknown mass and perturbations separately (instead, only the combined effect was analyzed).

In view of the preceding, the novel contribution of this work is further experimental validation of SAC theory in the application of spacecraft proximity operations and debris removal.

\subsection{Thesis Objectives}

This work experimentally validates adaptive control for trajectory tracking in spacecraft proximity operations and debris removal. Specifically, this work experimentally 
validates SAC theory for a chaser spacecraft performing a repeated circular trajectory around a target, where the initial tracking error is high and the chaser has an unknown mass and is subjected to perturbations. The combined effects of unknown mass and perturbations on trajectory tracking are investigated as well as each effect separately.

These experiments provide a more robust and realistic experimental validation of SAC theory than previous work. Simulations were also performed prior to each experiment.

New facilities were developed at Carleton University to perform the experiments in this work including an air bearing testbed and robotic platforms (representing spacecraft) to operate in this planar environment. The author contributed significantly to the development of these new facilities, notably creating the software environment necessary for simulations and experiments (see Sec. 5.2 for more details).

\subsection{Contributions}

The contributions of this work to the field of spacecraft proximity operations and debris removal are:

1. Designing and building an experimental facility to enable research for this work and future work related to spacecraft proximity operations and debris removal (see Sec. 5.2 for more details).

2. Providing further experimental validation of Simple Adaptive Control (SAC) for spacecraft trajectory tracking under uncertainties and perturbations by testing more complex trajectories and more rigorous conditions than previous work.

\subsection{Organization}

This work is organized into the following chapters:

Chapter 2: Relative Motion Dynamics presents the relative equations of motion between a chaser spacecraft and a target object, linearizes them and simplifies them to a double integrator model.

Chapter 3: Simple Adaptive Control presents the SAC theory used for trajectory 
tracking in this work's simulations and experiments.

Chapter 4: Simulations presents the simulation parameters and results.

Chapter 5: Experiments presents the experimental facility and the results obtained through experimental validation of SAC theory.

Chapter 6: Conclusion briefly emphasizes the most important results and describes possible future work. 


\section{Chapter 2}

\section{Relative Motion Dynamics}

Spacecraft formation flying is an important aspect of proximity operations. In these applications, it is preferable to define the relative position and velocity between the spacecraft in formation instead of using the inertial orbit as the reference. The purpose of this chapter is to describe the exact, nonlinear relative equations of motion and their linearized forms. All of the equations presented in this chapter were sourced from de Ruiter's textbook [12].

\subsection{Relative Orbit Geometry}

Typical formation flying involves a chaser spacecraft actively controlling its position and velocity relative to a passive target spacecraft. For example, this scenario describes rendezvous and docking maneuvers where the relative distance between both spacecraft is decreased in a slow cautious manner to prevent collision, or formation keeping maneuvers where the relative position between spacecraft must be kept constant.

The most basic scenario involves both spacecraft in identical orbits offset by a difference in their true anomalies. The relative distance will remain constant for circular orbits due to equal orbital speeds for both spacecraft whereas for elliptical orbits the relative distance will change according to the position within the orbit.

Formation flying becomes more difficult when the spacecraft have non-identical orbits. This scenario requires constraints to ensure bounded relative motion so the spacecraft do not drift away from each other and lose formation. 


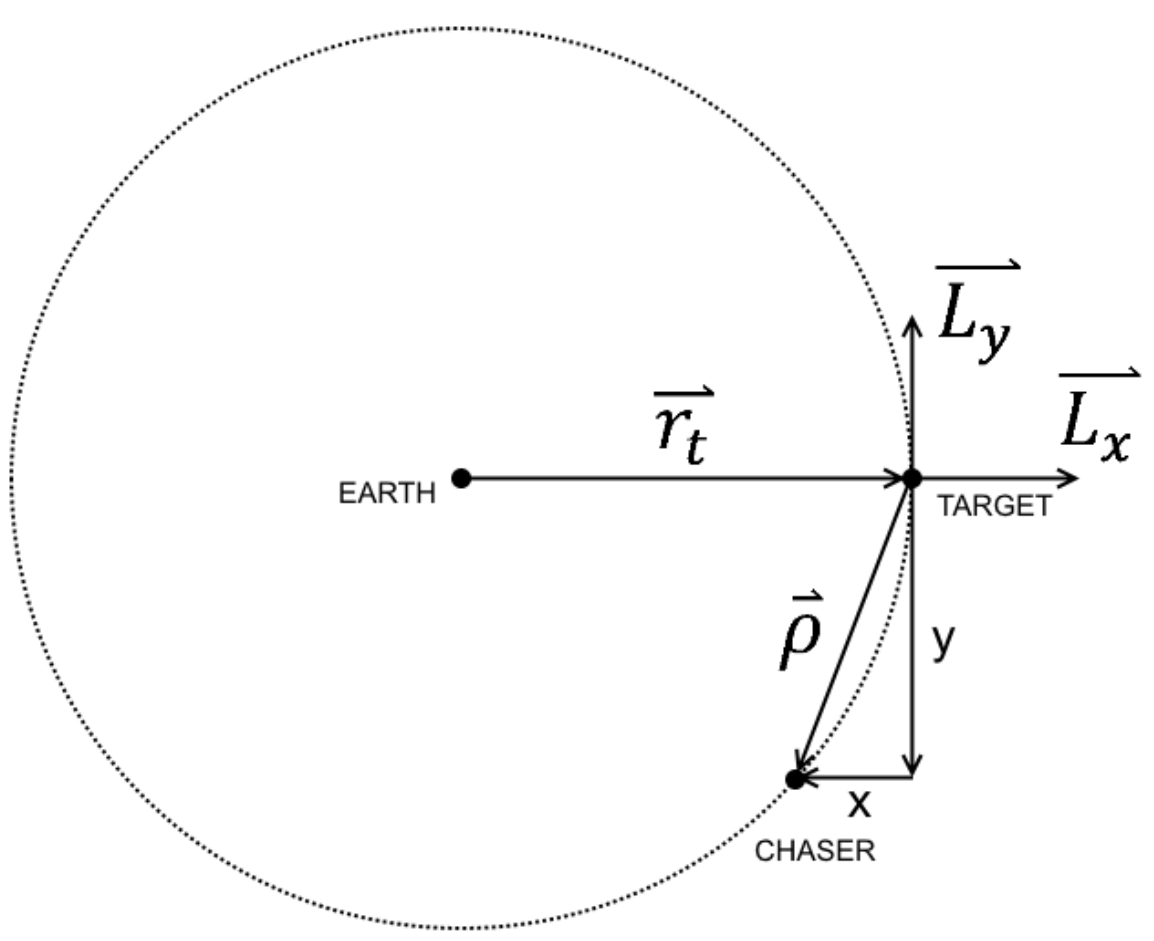

Figure 2.1: Local-vertical-local-horizontal reference frame.

\subsection{Nonlinear Relative Dynamics}

A moving reference frame titled Local-Vertical-Local-Horizontal (LVLH) and denoted $\mathcal{F}_{L}$ is introduced to develop the exact nonlinear equations for relative motion between a chaser and target spacecraft (shown in Fig. 2.1). This frame is defined by three unit vectors $\vec{L}_{x}, \vec{L}_{z}$ and $\vec{L}_{y}$ with the origin coinciding with the target spacecraft. The unit vectors $\vec{L}_{x}, \vec{L}_{z}$ and $\vec{L}_{y}$ are oriented towards the inertial target position vector $\vec{r}_{t}$, normal to the orbital plane and pointing along the orbital angular momentum vector $\vec{h}$, and in the orbital plane completing the right hand rule, respectively:

$$
\vec{L}_{x}=\vec{r}_{t} / r_{t} \quad \vec{L}_{z}=\vec{h} / h \quad \vec{L}_{y}=\vec{L}_{z} \times \vec{L}_{x}
$$

If the target is following a circular orbit then $\vec{L}_{y}$ points along its velocity vector. This frame rotates with the target spacecraft and is also known as Hill's frame. It can be expressed as a vectrix $\overrightarrow{\mathcal{F}}_{L}$, which is a matrix of unit vectors that describe a reference frame: 


$$
\overrightarrow{\mathcal{F}}_{L} \triangleq\left[\begin{array}{c}
\vec{L}_{x} \\
\vec{L}_{y} \\
\vec{L}_{z}
\end{array}\right]
$$

Fig. 2.1 illustrates the position vector of the chaser with respect to the target (denoted $\vec{\rho}$ ). The components of this vector in the LVLH frame can be expressed as follows:

$$
\vec{\rho}=\overrightarrow{\mathcal{F}}_{L}^{T}\left[\begin{array}{l}
x \\
y \\
z
\end{array}\right]
$$

where the in-plane motion (in the target orbital plane) is described by the $\{x, y\}$ components, titled radial and along-track, respectively, and the out-of-plane motion is described by the $z$ (cross-track) component.

The inertial position of the chaser can be determined according to the vector addition principle:

$$
\vec{r}_{c}=\vec{r}_{t}+\vec{\rho}
$$

where the inertial chaser position is denoted $\vec{r}_{c}$. Standard kinematics can be used to obtain the inertial chaser velocity $\left(\dot{\vec{r}}_{c}\right)$ and acceleration $\left(\ddot{\vec{r}}_{c}\right)$ :

$$
\begin{gathered}
\dot{\vec{r}}_{c}=\stackrel{\circ}{\vec{r}}_{c}+\vec{\omega}_{L I} \times \vec{r}_{c} \\
\ddot{\vec{r}}_{c}=\stackrel{\circ}{\vec{r}}_{c}+\dot{\vec{\omega}}_{L I} \times \vec{r}_{c}+2 \vec{\omega}_{L I} \times \stackrel{\circ}{\vec{r}}_{c}+\vec{\omega}_{L I} \times\left(\vec{\omega}_{L I} \times \vec{r}_{c}\right)
\end{gathered}
$$

where $\vec{\omega}_{L I}$ and $\dot{\vec{\omega}}_{L I}$ denote the angular velocity and acceleration of the LVLH frame $\mathcal{F}_{L}$ with respect to the inertial frame $\mathcal{F}_{I}$, respectively. Expressed in $\mathcal{F}_{L}$, the vectors from the above kinematics are as follows: 
$\vec{r}_{c}=\overrightarrow{\mathcal{F}}_{L}^{T} \mathbf{r}_{c_{L}}=\overrightarrow{\mathcal{F}}_{L}^{T}\left[\begin{array}{c}r_{t}+x \\ y \\ z\end{array}\right]$

$\stackrel{\circ}{\vec{r}}_{c}=\overrightarrow{\mathcal{F}}_{L}^{T} \dot{\mathbf{r}}_{c_{L}}=\overrightarrow{\mathcal{F}}_{L}^{T}\left[\begin{array}{c}\dot{r}_{t}+\dot{x} \\ \dot{y} \\ \dot{z}\end{array}\right]$

$\stackrel{\circ}{\vec{r}}_{c}=\overrightarrow{\mathcal{F}}_{L}^{T} \ddot{\mathbf{r}}_{c_{L}}=\overrightarrow{\mathcal{F}}_{L}^{T}\left[\begin{array}{c}\ddot{r}_{t}+\ddot{x} \\ \ddot{y} \\ \ddot{z}\end{array}\right]$

$\vec{\omega}_{L I}=\overrightarrow{\mathcal{F}}_{L}^{T} \boldsymbol{\omega}_{L I}=\overrightarrow{\mathcal{F}}_{L}^{T}\left[\begin{array}{c}0 \\ 0 \\ \dot{\theta}\end{array}\right]$

$$
\dot{\vec{\omega}}_{L I}=\overrightarrow{\mathcal{F}}_{L}^{T} \dot{\boldsymbol{\omega}}_{L I}=\overrightarrow{\mathcal{F}}_{L}^{T}\left[\begin{array}{l}
0 \\
0 \\
\ddot{\theta}
\end{array}\right]
$$

where the true anomaly of the target is denoted by $\theta$ and the vector magnitude of the target position is denoted by $r_{t}$. This allows the expression for acceleration to be rearranged into LVLH components as follows: 


$$
\begin{array}{r}
\ddot{\vec{r}}_{c}=\overrightarrow{\mathcal{F}}_{L}^{T}\left\{\left[\begin{array}{c}
\ddot{r}_{t}+\ddot{x} \\
\ddot{y} \\
\ddot{z}
\end{array}\right]+\left[\begin{array}{l}
0 \\
0 \\
\ddot{\theta}
\end{array}\right]\right. \\
\left.+\left[\begin{array}{c}
r_{t}+x \\
y \\
z
\end{array}\right]+2\left[\begin{array}{c}
0 \\
0 \\
\dot{\theta}
\end{array}\right] \begin{array}{c}
\dot{r}_{t}+\dot{x} \\
\dot{y} \\
\dot{z}
\end{array}\right] \\
\left.\left.+\left[\begin{array}{c}
0 \\
\dot{\theta}
\end{array}\right]^{\times}\left(\left[\begin{array}{c}
0 \\
0 \\
\dot{\theta}
\end{array}\right] \begin{array}{c}
r_{t}+x \\
y \\
z
\end{array}\right]\right)\right\} \\
\ddot{\vec{r}}_{c}=\overrightarrow{\mathcal{F}}_{L}^{T}\left[\begin{array}{c}
\ddot{r}_{t}+\ddot{x}-\ddot{\theta} y-2 \dot{\theta} \dot{y}-\dot{\theta}^{2}\left(r_{t}+x\right) \\
\ddot{y}+\ddot{\theta}\left(r_{t}+x\right)+2 \dot{\theta}\left(\dot{r}_{t}+\dot{x}\right)-\dot{\theta}^{2} y \\
\ddot{z}
\end{array}\right]
\end{array}
$$

The terms $\ddot{\theta}$ and $\ddot{r}_{t}$ can be replaced to further simplify the expression. Note that there is a relation between orbital position and angular momentum $h$ :

$$
h=r_{t}^{2} \dot{\theta}
$$

Taking the time derivative and assuming constant angular momentum $h$ (i.e. a Keplerian orbit) produces the following expression:

$$
\dot{h}=2 r_{t} \dot{r}_{t} \dot{\theta}+r_{t}^{2} \ddot{\theta}=0
$$

Now it is possible to solve for $\ddot{\theta}$ to produce the following expression:

$$
\ddot{\theta}=-2 \frac{\dot{r}_{t}}{r_{t}} \dot{\theta}
$$

The target position vector can be written in the LVLH frame according to its definition:

$$
\vec{r}_{t}=\overrightarrow{\mathcal{F}}_{L}^{T} \mathbf{r}_{t_{L}}=\overrightarrow{\mathcal{F}}_{L}^{T}\left[\begin{array}{c}
r_{t} \\
0 \\
0
\end{array}\right]
$$

Differentiating the above expression with respect to time twice while utilizing the fact 
that motion is constrained about the $\vec{L}_{x}$ axis produces the following expression:

$$
\ddot{\vec{r}}_{t}=\overrightarrow{\mathcal{F}}_{L}^{T}\left[\begin{array}{c}
\ddot{r}_{t}-\dot{\theta}^{2} r_{t} \\
0 \\
0
\end{array}\right]
$$

The target acceleration vector can be written in terms of LVLH components by using the so-called two-body equation of motion as follows:

$$
\ddot{\vec{r}}_{t}=-\overrightarrow{\mathcal{F}}_{L}^{T} \frac{\mu}{r_{t}^{3}}\left[\begin{array}{c}
r_{t} \\
0 \\
0
\end{array}\right]=-\overrightarrow{\mathcal{F}}_{L}^{T} \frac{\mu}{r_{t}^{2}}\left[\begin{array}{l}
1 \\
0 \\
0
\end{array}\right]
$$

Setting the above expressions equal to each other gives:

$$
\ddot{r}_{t}=\dot{\theta}^{2} r_{t}-\frac{\mu}{r_{t}^{2}}
$$

Now the acceleration vector of the chaser can be written in a simpler form by substituting $\ddot{\theta}$ and $\ddot{r}_{t}$ into Eq. (2.13):

$$
\ddot{\vec{r}}_{c}=\overrightarrow{\mathcal{F}}_{L}^{T}\left[\begin{array}{c}
\ddot{x}-\dot{\theta}^{2} x-2 \dot{\theta}\left(\dot{y}-y \frac{\dot{r}_{t}}{r_{t}}\right)-\frac{\mu}{r_{t}^{2}} \\
\ddot{y}-\dot{\theta}^{2} y+2 \dot{\theta}\left(\dot{x}-x \frac{\dot{r}_{t}}{r_{t}}\right) \\
\ddot{z}
\end{array}\right]
$$

The chaser acceleration vector can also be written in terms of LVLH components by using the two-body equation as follows:

$$
\ddot{\vec{r}}_{c}=-\overrightarrow{\mathcal{F}}_{L}^{T} \frac{\mu}{r_{c}^{3}}\left[\begin{array}{c}
r_{t}+x \\
y \\
z
\end{array}\right]
$$

The last step to arrive at the exact, nonlinear relative equations of motion for the chaser acceleration relative to the target (in the LVLH frame) is to set Eqs. (2.21) and (2.22) equal to each other: 


$$
\begin{aligned}
\ddot{x}-\dot{\theta}^{2} x-2 \dot{\theta}\left(\dot{y}-y \frac{\dot{r}_{t}}{r_{t}}\right)-\frac{\mu}{r_{t}^{2}} & =-\frac{\mu}{r_{c}^{3}}\left(r_{t}+x\right) \\
\ddot{y}-\dot{\theta}^{2} y+2 \dot{\theta}\left(\dot{x}-x \frac{\dot{r}_{t}}{r_{t}}\right) & =-\frac{\mu}{r_{c}^{3}} y \\
\ddot{z} & =-\frac{\mu}{r_{c}^{3}} z
\end{aligned}
$$

If the position of the chaser relative to the target is assumed to be negligible compared to the target orbital radius $r_{t}$ then Eqs. (2.23) to (2.25) can be simplified further. The orbital radius of the chaser $r_{c}$ becomes:

$$
\begin{aligned}
r_{c} & =\sqrt{\left(r_{t}+x\right)^{2}+y^{2}+z^{2}} \\
& =\sqrt{r_{t}^{2}+2 r_{t} x+x^{2}+y^{2}+z^{2}} \\
& =r_{t} \sqrt{1+2 \frac{x}{r_{t}}+\frac{x^{2}+y^{2}+z^{2}}{r_{t}^{2}}}
\end{aligned}
$$

which is approximately

$$
r_{c} \approx r_{t} \sqrt{1+2 \frac{x}{r_{t}}}
$$

which allows the term $\frac{1}{r_{c}^{3}}=r_{c}^{-3}$ in Eqs. (2.23) to (2.25) to be expressed as follows:

$$
r_{c}^{-3} \approx r_{t}^{-3}\left(1+2 \frac{x}{r_{t}}\right)^{-3 / 2}
$$

Recalling the binomial expansion theorem:

$$
(a+b)^{n}=a^{n}+n a^{n-1} b+\frac{n(n-1)}{2 !} a^{n-2} b^{2}+\frac{n(n-1)(n-2)}{3 !} a^{n-3} b^{3}+\ldots
$$

This allows Eq. (2.30) to be rewritten as: 


$$
\begin{aligned}
r_{c}^{-3} & \approx r_{t}^{-3}\left\{1-\frac{3}{2}\left(2 \frac{x}{r_{t}}\right)\right\} \\
& \approx r_{t}^{-3}\left\{1-\frac{3 x}{r_{t}}\right\}
\end{aligned}
$$

Now the chaser acceleration LVLH components (Eq. (2.22)) can be simplified using the above approximation:

$$
\begin{aligned}
-\frac{\mu}{r_{c}^{3}}\left[\begin{array}{c}
r_{t}+x \\
y \\
z
\end{array}\right] & \approx-\frac{\mu}{r_{t}^{3}}\left\{1-\frac{3 x}{r_{t}}\right\}\left[\begin{array}{c}
r_{t}+x \\
y \\
z
\end{array}\right] \\
& \approx-\frac{\mu}{r_{t}^{3}}\left[\begin{array}{c}
\left(1-\frac{3 x}{r_{t}}\right)\left(r_{t}+x\right) \\
\left(1-\frac{3 x}{r_{t}}\right) y \\
\left(1-\frac{3 x}{r_{t}}\right) z
\end{array}\right]
\end{aligned}
$$

If higher order terms are considered negligible, the above expression simplifies further:

$$
-\frac{\mu}{r_{c}^{3}}\left[\begin{array}{c}
r_{t}+x \\
y \\
z
\end{array}\right] \approx-\frac{\mu}{r_{t}^{3}}\left[\begin{array}{c}
r_{t}-2 x \\
y \\
z
\end{array}\right]
$$

Finally the general equations of relative motion given by Eqs. (2.23) to (2.25) can be expressed in a simplified form by using the above result and Eq. (2.16):

$$
\begin{aligned}
\ddot{x}-x\left(\dot{\theta}^{2}+2 \frac{\mu}{r_{t}^{3}}\right)-2 \dot{\theta} \dot{y}-\ddot{\theta} y & =0 \\
\ddot{y}-y\left(\dot{\theta}^{2}-\frac{\mu}{r_{t}^{3}}\right)+2 \dot{\theta} \dot{x}+\ddot{\theta} x & =0 \\
\ddot{z}+\frac{\mu}{r_{t}^{3}} z & =0
\end{aligned}
$$




\subsection{Linearized Relative Dynamics}

In a circular orbit, the orbital radius $r_{t}$ and true anomaly rate $\dot{\theta}$ are constant, and

the true anomaly rate $\dot{\theta}$ and mean orbital motion $n$ are equivalent. Therefore if the target orbit is approximately circular, the relative equations of motion (Eqs. (2.38) to $(2.40))$ can be simplified to linear forms (Hill's equations):

$$
\begin{aligned}
\ddot{x}-3 n^{2} x-2 n \dot{y} & =0 \\
\ddot{y}+2 n \dot{x} & =0 \\
\ddot{z}+n^{2} z & =0
\end{aligned}
$$

Two assumptions must hold for Hill's equations to be valid: (1) the target orbit must be circular and (2) the relative distance between target and chaser must be negligible compared to the radius of their orbits.

\subsection{Double Integrator Model}

Hill's equations are often valid for proximity operations between a target and chaser spacecraft since the necessary assumptions - the target orbit being approximately circular and the the relative distance between target and chaser being small compared to their orbital radii - hold true for many scenarios. There are additional criteria which can simplify the dynamics even further: if the time scale of the proximity operations is small relative to the orbital period and the distance between the target and chaser is no more than a few meters, the mean orbital motion $n$ becomes negligible compared to the other terms in Hill's equations and they can be simplified to an un-coupled double integrator model [6] as follows:

$$
\begin{aligned}
& \ddot{x}=0 \\
& \ddot{y}=0 \\
& \ddot{z}=0
\end{aligned}
$$


Note that the time scale of the proximity operations being small relative to the orbital period depends largely on the orbital altitude. The orbital period increases with orbital altitude according to Kepler's third law:

$$
T=\sqrt{\frac{4 \pi^{2}}{\mu} a^{3}}
$$

where $T$ is the orbital period, $\mu$ is the standard gravitational parameter (constant for Earth) and $a$ is the semi-major axis of the orbit, or simply the orbital radius for a circular orbit. Recall that the orbital radius is the addition of the planet's radius and the orbital altitude above the planet's surface. Higher altitude orbits have longer periods making a given time scale for proximity operations relatively smaller as altitude increases. Low Earth orbits (LEO) typically have periods of 90 minutes, Geostationary Earth orbits (GEO) by definition are synchronized with the Earth's rotation and have 24 hour periods and higher altitude orbits have even longer periods. In all cases, the orbital period $T$ is large enough to render the mean orbital motion $n$ negligible in Eqs. (2.41) to (2.43) [6]. By definition, the mean orbital motion is:

$$
n \triangleq \frac{2 \pi}{T}
$$

therefore the longer the orbital period $T$, the smaller the mean orbital motion $n$ making it even less significant compared to the other terms in in Eqs. (2.41) to (2.43). Including forces for the active chaser spacecraft in Eqs. (2.44) to (2.46) and neglecting out-of-plane motion, the equations become:

$$
\begin{aligned}
& \ddot{x}=\frac{f_{x}}{m} \\
& \ddot{y}=\frac{f_{y}}{m}
\end{aligned}
$$

where $f_{x}$ and $f_{y}$ represent force components applied to the chaser spacecraft in the $x$ and $y$ directions, respectively, and $m$ represents the mass of the chaser spacecraft. Note that Eqs. (2.49) and (2.50) are used for the dynamics in simulations described in Chapter 4 which have two-dimensional motion in a planar environment (3DOF 
including attitude). The real space environment has 6DOF (rotational and translational motion in the $x, y$ and $z$ directions) but simulations and experiments in this work are restricted to 3DOF because experiments are meant to validate simulation results and the experimental facility only allows for planar motion.

\subsection{State Space Representation}

Finally, the linearized uncoupled double integrator model can be expressed as a square state-space system by defining the state $\boldsymbol{y} \in \mathbb{R}^{3}$ as the sum of position and velocity, $\boldsymbol{x}=\left[\begin{array}{llllll}x & y & z & \dot{x} & \dot{y} & \dot{z}\end{array}\right]^{T} \in \mathbb{R}^{6}$, and the control inputs as $\boldsymbol{u}=\left[\begin{array}{ccc}f_{x} & f_{y} & f_{z}\end{array}\right]^{T} \in$ $\mathbb{R}^{3}$ :

$$
\dot{\boldsymbol{x}}=\boldsymbol{A x}+\boldsymbol{B u}, \quad \boldsymbol{y}=\boldsymbol{C x}
$$

where $\left[\begin{array}{ccc}x & y & z\end{array}\right]^{T} \in \mathbb{R}^{3}$ and $\left[\begin{array}{ccc}\dot{x} & \dot{y} & \dot{z}\end{array}\right]^{T} \in \mathbb{R}^{3}$ are relative position and velocity in the LVLH frame. Recall that this frame has its $x$ axis aligned with the orbital radius vector of the target, its $z$ axis aligned with the orbital angular momentum vector of the target, and its $y$ axis completing the right hand rule as in Fig. 2.1. Recall that for this work the $z$ dimension is not used due to the planar environment of the experimental facility and the corresponding simulations. The product $\boldsymbol{C} \boldsymbol{x}$ yields the state vector $\boldsymbol{y}$ which contains the sums of position and velocity:

$$
\boldsymbol{y}=\left[\begin{array}{c}
x+\dot{x} \\
y+\dot{y} \\
z+\dot{z}
\end{array}\right]
$$

Representing the double integrator model as a square state-space system is important for the stability of the adaptive controller as described in Sec. 3.3. 


\section{Chapter 3}

\section{Simple Adaptive Control}

This chapter describes Simple Adaptive Control (SAC) theory by presenting the ideal model, specifying the control objectives, and describing the control law and its control gain adaptation mechanisms.

\subsection{Ideal Model}

SAC relies upon an ideal model to perform control. The control objective is to force the uncertain outputs to asymptotically track the ideal outputs, as specified by the ideal model. The ideal model can be described linearly as follows:

$$
\dot{\boldsymbol{x}}_{m}=\boldsymbol{A}_{m} \boldsymbol{x}_{m}+\boldsymbol{B}_{m} \boldsymbol{u}_{m}, \quad \boldsymbol{y}_{m}=\boldsymbol{C}_{m} \boldsymbol{x}_{m}
$$

where $\boldsymbol{x}_{m}, \boldsymbol{y}_{m}$ and $\boldsymbol{u}_{m}$ denote the ideal model's state vector, the ideal model output and the ideal model input, respectively. The ideal model's state vector is defined as follows:

$$
\boldsymbol{x}_{m}=\left[\begin{array}{llllll}
x_{m} & y_{m} & z_{m} & \dot{x}_{m} & \dot{y}_{m} & \dot{z}_{m}
\end{array}\right]^{T} \in \mathbb{R}^{6}
$$

and the input vector to the ideal model is defined as follows:

$$
\boldsymbol{u}_{m}=\left[\begin{array}{lll}
x_{d} & y_{d} & z_{d}
\end{array}\right]^{T} \in \mathbb{R}^{3}
$$

where the desired relative trajectory is denoted by $x_{d}, y_{d}, z_{d} \in \mathbb{R}$ and the ideal model output is denoted by $\boldsymbol{y}_{m} \in \mathbb{R}^{3}$. The desired response for relative motion can be described using the ideal damping ratio $\zeta \in \mathbb{R}$ and the undamped natural frequency $\omega_{n} \in \mathbb{R}$ in the following expression: 


$$
\begin{array}{r}
\boldsymbol{A}_{m}=\left[\begin{array}{cc}
\mathbf{0}_{3 \times 3} & \boldsymbol{I}_{3 \times 3} \\
-\omega_{n}^{2} \boldsymbol{I}_{3 \times 3} & -2 \zeta \omega_{n} \boldsymbol{I}_{3 \times 3}
\end{array}\right] \in \mathbb{R}^{6 \times 6}, \quad \boldsymbol{B}_{m}=\left[\begin{array}{c}
\mathbf{0}_{3 \times 3} \\
\omega_{n}^{2} \boldsymbol{I}_{3 \times 3}
\end{array}\right] \in \mathbb{R}^{6 \times 3}, \\
\boldsymbol{C}_{m}=\left[\begin{array}{ll}
\alpha_{m} \boldsymbol{I}_{3 \times 3} & \boldsymbol{I}_{3 \times 3}
\end{array}\right] \in \mathbb{R}^{3 \times 6}
\end{array}
$$

Note that the undamped natural frequency $\omega_{n}$ is driven by the desired settling time $t_{s}$ and ideal damping ratio $\zeta$ :

$$
\omega_{n}=\frac{4}{t_{s} \zeta}
$$

Also note that the ideal model presented here only describes the desired relative motion and contains no information about the plant or dynamics parameters, as was established in Section 1.3 as a computationally efficient method suitable for onboard processors in spacecraft applications.

\subsection{Control Objective}

The control objective must be accomplished without information about the target spacecraft mass $m$ or any perturbations present, as described in Section 1.2. Therefore the uncertainties are in the input matrix $\boldsymbol{B}$ and control input matrix $\boldsymbol{u}$. An output tracking error $\boldsymbol{e}_{y} \in \mathbb{R}^{3}$ allows the control objective to be quantitatively measured:

$$
\boldsymbol{e}_{y} \triangleq \boldsymbol{y}_{m}-\boldsymbol{y}
$$

\subsection{Control Law and Adaptation Mechanisms}

The controller is capable of employing a direct adaptation law for real-time adjustment of control gains according the tracking error $\boldsymbol{e}_{y}$. This solves the problem of the uncertainties and, unlike indirect adaptive control, does not require real-time estimation of uncertainties. The adaptive control method is based upon the command generator tracker method [13] and makes use of all data regarding the ideal model; that is the adaptive control method determines control input forces by using the 
model states and inputs implemented in a feed-forward model configuration, as per SAC theory [10]:

$$
\boldsymbol{u}(t)=\boldsymbol{K}_{e}(t) \boldsymbol{e}_{y}(t)+\boldsymbol{K}_{x}(t) \boldsymbol{x}_{m}(t)+\boldsymbol{K}_{u}(t) \boldsymbol{u}_{m}(t)
$$

where $\boldsymbol{K}_{e}(t) \in \mathbb{R}^{3 \times 3}$ is the time-varying stabilizing control gain matrix, and $\boldsymbol{K}_{x}(t) \in$ $\mathbb{R}^{3 \times 6}$ and $\boldsymbol{K}_{u}(t) \in \mathbb{R}^{3 \times 3}$ are time-varying feed-forward control gains that help reduce tracking error. It should be noted for clarification purposes that $\boldsymbol{u}$ is the vector containing the time-varying control forces (similarly to Eqn. 4.1 but produced by $\mathrm{SAC}$ instead of $\mathrm{PD}$ control), that is:

$$
\boldsymbol{u}=\boldsymbol{f}_{\text {control }}=\left[\begin{array}{c}
f_{x} \\
f_{y} \\
f_{z}
\end{array}\right]
$$

In practice both $\boldsymbol{K}_{x}(t)$ and $\boldsymbol{K}_{u}(t)$ enable good tracking performance without the need for a large stabilizing gain $\boldsymbol{K}_{e}(t)$. An adaptive algorithm is responsible for setting these gains and asymptotically reducing the tracking error to zero. This algorithm makes use of the tracking error $\boldsymbol{e}_{y}$ to determine the integral components of the gains to maximize the steepness of the tracking error reduction:

$$
\begin{aligned}
\dot{\boldsymbol{K}}_{I x}(t) & =\boldsymbol{e}_{y} \boldsymbol{x}_{m}^{T} \boldsymbol{\Gamma}_{I x} \\
\dot{\boldsymbol{K}}_{\text {Iu }}(t) & =\boldsymbol{e}_{y} \boldsymbol{u}_{m}^{T} \boldsymbol{\Gamma}_{I u}
\end{aligned}
$$

where $\Gamma_{I u} \in \mathbb{R}^{3 \times 3}$ and $\Gamma_{I x} \in \mathbb{R}^{6 \times 6}$ are positive-definite diagonal matrices. These matrices determine the adaptation rate of the control gains. An integral component of the error adaptive control gain is included:

$$
\dot{\boldsymbol{K}}_{I e}(t)=\boldsymbol{e}_{y} \boldsymbol{e}_{y}^{T} \boldsymbol{\Gamma}_{I e}
$$

where $\boldsymbol{\Gamma}_{I e} \in \mathbb{R}^{3 \times 3}$ is a constant matrix that determines the adaptation rate of $\boldsymbol{K}_{I e}(t)$. In a non-ideal tracking scenario (for example caused by measurement noise), this 
integral gain could become too large because it corresponds to a monotonically increasing term. Section 3.5 describes a solution to this problem where a forgetting factor is included in the adaptation mechanism to slowly reduce the integral gains in the absence of large tracking errors so the integral gains do not grow out of control.

To make convergence towards perfect tracking even faster, proportional adaptive control terms are included as in [11]. The proportional terms are obtained similarly to the integral terms:

$$
\begin{aligned}
\boldsymbol{K}_{P e}(t) & =\boldsymbol{e}_{y} \boldsymbol{e}_{y}^{T} \boldsymbol{\Gamma}_{P e} \\
\boldsymbol{K}_{P x}(t) & =\boldsymbol{e}_{y} \boldsymbol{x}_{m}^{T} \boldsymbol{\Gamma}_{P x} \\
\boldsymbol{K}_{P u}(t) & =\boldsymbol{e}_{y} \boldsymbol{u}_{m}^{T} \boldsymbol{\Gamma}_{P u}
\end{aligned}
$$

where $\boldsymbol{\Gamma}_{P e}, \boldsymbol{\Gamma}_{P u} \in \mathbb{R}^{3 \times 3}$ and $\boldsymbol{\Gamma}_{P x} \in \mathbb{R}^{6 \times 6}$ are the constant coefficient matrices. Finally the integral and proportional terms are summed together to obtain the total adaptive gains:

$$
\begin{aligned}
\boldsymbol{K}_{e}(t) & =\boldsymbol{K}_{P e}(t)+\boldsymbol{K}_{I e}(t) \\
\boldsymbol{K}_{x}(t) & =\boldsymbol{K}_{P x}(t)+\boldsymbol{K}_{I x}(t) \\
\boldsymbol{K}_{u}(t) & =\boldsymbol{K}_{P u}(t)+\boldsymbol{K}_{I u}(t)
\end{aligned}
$$

Now the adaptive algorithm can simply be expressed as follows:

$$
\boldsymbol{u}=\boldsymbol{K}(t) \boldsymbol{r}
$$

where the terms $\boldsymbol{K}(t) \in \mathbb{R}^{3 \times 12}$ and $\boldsymbol{r} \in \mathbb{R}^{12 \times 1}$ are defined as follows:

$$
\begin{aligned}
\boldsymbol{K}(t) & =\left[\begin{array}{lll}
\boldsymbol{K}_{e}(t) & \boldsymbol{K}_{x}(t) & \boldsymbol{K}_{u}(t)
\end{array}\right]=\boldsymbol{K}_{P}(t)+\boldsymbol{K}_{I}(t) \\
\boldsymbol{r} & =\left[\begin{array}{lll}
\boldsymbol{e}_{y}^{T} & \boldsymbol{x}_{m}^{T} & \boldsymbol{u}_{m}^{T}
\end{array}\right]^{T}
\end{aligned}
$$


With the introduction of the above equations, $\boldsymbol{K}_{P}(t), \boldsymbol{K}_{I}(t) \in \mathbb{R}^{3 \times 12}$ can now be expressed as follows:

$$
\begin{gathered}
\boldsymbol{K}_{P}(t)=\boldsymbol{e}_{y} \boldsymbol{r}^{T} \boldsymbol{\Gamma}_{P} \\
\dot{\boldsymbol{K}}_{I}(t)=\boldsymbol{e}_{y} \boldsymbol{r}^{T} \boldsymbol{\Gamma}_{I}
\end{gathered}
$$

where $\boldsymbol{\Gamma}_{P}, \boldsymbol{\Gamma}_{I} \in \mathbb{R}^{12 \times 12}$ are the adaptation coefficient matrices for the combined gains $\boldsymbol{K}_{p}(t)$ and $\boldsymbol{K}_{I}(t)$ as in the following:

$$
\boldsymbol{\Gamma}_{P}=\left[\begin{array}{ccc}
\boldsymbol{\Gamma}_{P e} & & \\
& \boldsymbol{\Gamma}_{P x} & \\
& & \boldsymbol{\Gamma}_{P u}
\end{array}\right], \quad \boldsymbol{\Gamma}_{I}=\left[\begin{array}{ccc}
\boldsymbol{\Gamma}_{I e} & & \\
& \boldsymbol{\Gamma}_{I x} & \\
& & \boldsymbol{\Gamma}_{I u}
\end{array}\right]
$$

This adaptation law has been shown to be asymptotically stable by Ulrich et al [11]. One of the stability requirements for SAC is that the product $\mathrm{CB}$ must be positive symmetric. This is why the dynamics are represented as a square state-space system in Sec. 2.5. The number of inputs is equal to the number of outputs to ensure that the state-space system is square. Position and velocity terms are summed together such that the $\boldsymbol{y}$ vector matches the size of the control inputs vector, instead of dropping the velocity terms to make the vectors match (the velocity terms are needed to perform damping).

\subsection{Ideal Control and State Trajectory}

According to command generator tracker methodology [13], when perfect tracking occurs - that is when the uncertain plant outputs (Eq. (2.51)) track the ideal model outputs without error - the plant is considered to be moving along the bounded ideal state trajectory $\boldsymbol{x}^{*} \in \mathbb{R}^{6}$. Therefore the dynamics of the plant under this ideal condition can be expressed as follows:

$$
\dot{x}^{*}=A x^{*}+B u^{*}, \quad y^{*}=C x^{*}
$$

where $\boldsymbol{u}^{*}$ and $\boldsymbol{y}^{*}$ denote the ideal input and output, respectively. It is important to 
distinguish between the ideal state $\boldsymbol{x}^{*}$ and the ideal model state $\boldsymbol{x}_{m}$ because the order of $\boldsymbol{x}^{*}$ is always equal to the order of the plant $\boldsymbol{x}$ (which could be large) but the order of $\boldsymbol{x}_{m}$ just has to be large enough to produce the desired output signal $\boldsymbol{y}_{m}$. The ideal control law, denoted by $\boldsymbol{u}^{*} \in \mathbb{R}^{3}$, is given by:

$$
\boldsymbol{u}^{*}=\tilde{\boldsymbol{K}}_{e} \boldsymbol{e}_{y}+\tilde{\boldsymbol{K}}_{x} \boldsymbol{x}_{m}+\tilde{\boldsymbol{K}}_{u} \boldsymbol{u}_{m}
$$

The constant ideal stabilizing gain matrix, denoted by $\tilde{\boldsymbol{K}}_{e} \in \mathbb{R}^{3 \times 3}$, is analogous to the adaptive control gains from the actual control law (Eq. (3.7)). The constant ideal control gain matrices $\tilde{\boldsymbol{K}}_{x} \in \mathbb{R}^{3 \times 6}$ and $\tilde{\boldsymbol{K}}_{u} \in \mathbb{R}^{3 \times 3}$ are for the feedforward configuration. Note that the ideal control gains represent constant values needed for perfect tracking and are never implemented. Under ideal tracking, the output tracking error vanishes, that is:

$$
\boldsymbol{e}_{y}=\boldsymbol{y}_{m}-\boldsymbol{y}=\mathbf{0}_{3}
$$

In the above case, $\boldsymbol{u}^{*}$ can be reduced to:

$$
\boldsymbol{u}^{*}=\tilde{\boldsymbol{K}}_{x} \boldsymbol{x}_{m}+\tilde{\boldsymbol{K}}_{u} \boldsymbol{u}_{m}
$$

In all other cases there is a state error, denoted by $\boldsymbol{e}_{x} \in \mathbb{R}^{6}$ and expressed as follows:

$$
e_{x} \triangleq x^{*}-x
$$

When there is a state error there must also be an output tracking error, denoted by $\boldsymbol{e}_{y}$ and expressed as follows:

$$
e_{y}=C \boldsymbol{x}^{*}-C \boldsymbol{x}=\boldsymbol{C e _ { x }}
$$

\subsection{Tracking under Non-ideal Conditions}

A classic problem with the original adaptive control law is that under non-ideal conditions, the integral time-varying control gain $\boldsymbol{K}_{I}(t)$ could cause divergence. One way to solve this and avoid divergence is the $\sigma$-modification introduced by Narendra et 
al. [14]. This $\sigma$-modification was then made popular by the work of Narendra and Annaswamy [15], Ioannou and Kokotovic [16,17] and Barkana and Kaufman [18,19]. SAC had its first $\sigma$-modification proposed by Fradkov [20]. The modification is made by including additional terms (commonly referred to as forgetting terms or forgetting factors) in the integral gain adaptation mechanisms. Using this modification, the time-varying integral control gains become:

$$
\begin{gathered}
\dot{\boldsymbol{K}}_{I_{e}}(t)=\boldsymbol{e}_{y} \boldsymbol{e}_{y}^{T} \boldsymbol{\Gamma}_{I_{e}}-\sigma_{e} \boldsymbol{K}_{I_{e}}(t) \\
\dot{\boldsymbol{K}}_{I_{x}}(t)=\boldsymbol{e}_{y} \boldsymbol{x}_{m}^{T} \boldsymbol{\Gamma}_{I_{x}}-\sigma_{x} \boldsymbol{K}_{I_{x}}(t) \\
\dot{\boldsymbol{K}}_{I_{u}}(t)=\boldsymbol{e}_{y} \boldsymbol{u}_{m}^{T} \boldsymbol{\Gamma}_{I_{u}}-\sigma_{u} \boldsymbol{K}_{I_{u}}(t)
\end{gathered}
$$

now combining the above expressions:

$$
\dot{\boldsymbol{K}}_{I}(t)=\boldsymbol{e}_{y} \boldsymbol{r}^{T} \boldsymbol{\Gamma}_{I}-\boldsymbol{\sigma} \boldsymbol{K}_{I}(t)
$$

where

$$
\boldsymbol{\sigma}=\left[\begin{array}{ccc}
\sigma_{e} & 0 & 0 \\
0 & \sigma_{x} & 0 \\
0 & 0 & \sigma_{u}
\end{array}\right] \in \mathbb{R}^{3 \times 3}
$$

and where the forgetting factors denoted by $\sigma_{e}, \sigma_{x}, \sigma_{u}$ are scalars and $\boldsymbol{\sigma} \in \mathbb{R}^{3 \times 3}$ is a matrix with the forgetting factors along its diagonal. Adjusting the algorithm to include these forgetting factors is advantageous in practical applications with noisy measurements because they prevent the integral adaptive gains from endlessly increasing due to tracking error that never perfectly converges to zero. The integral gains are important for good controller performance and can help reduce tracking error but if that error is not reduced to zero (for example because of measurement noise) and there is no forgetting factor then the integral gains can increase to the point where they diverge or negatively impact tracking performance. The forgetting factor should 
be chosen so that it allows the integral gains to grow when needed (when the tracking error is large) but also allows the integral gains to decrease when the tracking error is small and primarily caused by measurement noise. One potential disadvantage of the forgetting factor once tracking error has been mostly eliminated is that it could cause the integral gains to become too small, however this is a self-correcting problem as the increased tracking error resulting from insufficiently large integral gains will cause those gains to grow in response (and reduce the tracking error again). This effect is called error bursting. The benefits of including the forgetting factor to prevent excessive integral gains outweigh this effect. 


\section{Chapter 4}

\section{Simulations}

This chapter presents numerical simulations of a chaser spacecraft tracking a circular inspection trajectory around a target in the context of space debris removal and proximity operations. The simulations conform to the experimental facility described in Chapter 5 in which a chaser platform performs trajectory tracking in a frictionless planar environment: the chaser platform floats and propels itself on an air bearing testbed (a large smooth granite table).

\subsection{Numerical Simulations}

An active control strategy is required for the chaser spacecraft to successfully track a circular inspection trajectory around target debris. A proportional-derivative (PD) controller was compared against an adaptive controller (SAC) for this purpose under a total of four sets of conditions:

1. Nominal (no perturbations other than the table slope, and the chaser's mass is known)

2. The addition of an unknown mass to the chaser

3. Subjecting the chaser to additional perturbation forces

4. Fully off-nominal (perturbations and the addition of an unknown mass to the chaser)

Comparing PD control against SAC for each set of conditions resulted in a total of eight scenarios being tested in simulation using MATLAB/Simulink prior to experiments. Note that the perturbations present in simulation were not the same as those in experiments (see Sec. 5.4 for more details). The following parameters were used: 
Table 4.1: Initial conditions for simulations and experiments.

\begin{tabular}{cc}
\hline \hline Parameter & Value \\
\hline$J_{z z, n o m}, \mathrm{~kg} \cdot \mathrm{m}^{2}$ & 0.30 \\
$J_{z z, o f f}, \mathrm{~kg} \cdot \mathrm{m}^{2}$ & 0.36 \\
$m_{\text {nom }}, \mathrm{kg}$ & 17.24 \\
$m_{\text {off }}, \mathrm{kg}$ & 26.37 \\
$\mathbf{r}, \mathrm{m}$ & {$[1.752 ; 0.756]$} \\
$\theta, \mathrm{deg}$ & 90 \\
Timestep, s & 0.002 \\
Experiment and simulation duration, s & 180 \\
$\mathbf{r}_{t}, \mathrm{~m}$ & {$[1.752 ; 1.206]$} \\
$R_{t}, \mathrm{~m}$ & 0.8 \\
Acceleration due to table slope, $/ \mathrm{s}^{2}$ & {$[-0.0003 ; 0.001]$} \\
$K_{P, \theta}$ & 0.33 \\
$K_{D, \theta}$ & 0.56 \\
$\mathbf{K}_{P}$ & $0.023 \mathbf{I}_{2 \times 2}$ \\
$\mathbf{K}_{D}$ & $0.065 \mathbf{I}_{2 \times 2}$ \\
$t_{s}, \mathrm{~s}$ & 12 \\
$\zeta$ & 0.9 \\
$\alpha_{m}$ & 1 \\
$\boldsymbol{\Gamma}_{P e}$ & $1.0 e-5 \mathbf{I}_{3 \times 3}$ \\
$\boldsymbol{\Gamma}_{P x}$ & $8.0 e-9 \mathbf{I}_{6 \times 6}$ \\
$\boldsymbol{\Gamma}_{P u}$ & $8.0 e-9 \mathbf{I}_{3 \times 3}$ \\
$\boldsymbol{\Gamma}_{I e}$ & $1.0 e-5 \mathbf{I}_{3 \times 3}$ \\
$\boldsymbol{\Gamma}_{I x}$ & $1.0 e-11 \mathbf{I}_{6 \times 6}$ \\
$\boldsymbol{\Gamma}_{I u}$ & $1.0 e-11 \mathbf{I}_{3 \times 3}$ \\
$\sigma_{e}, \sigma_{x}, \sigma_{u}$ & 0.4 \\
\hline \hline
\end{tabular}

where $R_{t}$ and $\mathbf{r}_{t}$ are the radius and center position of the commanded circular trajectory, respectively, $\mathbf{r}$ is the initial position of the chaser, $m_{n o m}$ and $m_{\text {off }}$ are the nominal and off-nominal masses of the chaser, respectively, $J_{z z, n o m}$ and $J_{z z, \text { off }}$ are the nominal and off-nominal moments of inertia of the chaser, respectively, and all positions and accelerations are given in the table frame (a reference frame with its origin at the corner of the air bearing testbed) as defined in Fig. 5.5. The origin is located at the top-left corner of the table, with the positive $x$ axis pointing down and the positive $y$ axis pointing to the right, and $\theta$ is the initial angular position of the chaser, measured positive counter-clockwise from the $x$ axis of the table frame to the 
$x$ axis of the chaser frame (the chaser's $x$ axis points in the direction of its cameras or 'eyes'). In simpler terms, the chaser is initially looking at the target debris and continues to look at it during the entire trajectory. Also note that the friction between the chaser and the table is negligible. The parameters in Table 4.1 are visually represented in Fig. 4.1 for clarification purposes:

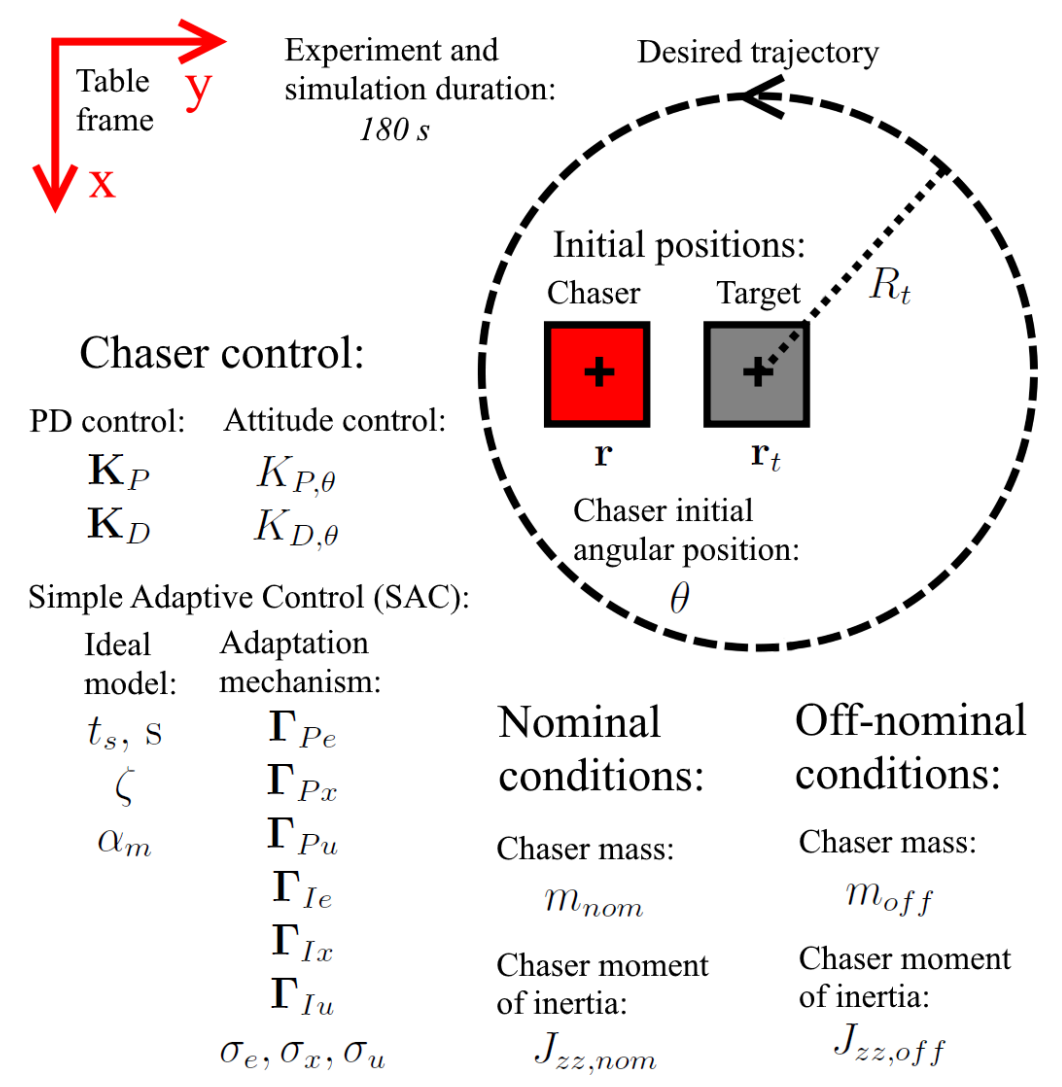

Figure 4.1: Visual representation of parameters used in simulations and experiments

A fixed timestep of 0.002 seconds was used along with the Euler method for numerical integration. Every simulation and experiment had a duration of $180 \mathrm{~s}$. The settling time, $t_{s}$, and ideal damping ratio, $\zeta$, are used for the adaptive controller's ideal model to determine the undamped natural frequency, $\omega_{n}$. The scaling factor $\alpha_{m}$ scales the output of the ideal model (set to 1 for no scaling). The adaptation coefficient matrices $\boldsymbol{\Gamma}_{P e}, \boldsymbol{\Gamma}_{P x}, \boldsymbol{\Gamma}_{P u}$ control the adaptation of the proportional timevarying control gain $\boldsymbol{K}_{P}(t)$ and $\boldsymbol{\Gamma}_{I e}, \boldsymbol{\Gamma}_{I x}, \boldsymbol{\Gamma}_{I u}$ control the adaptation of the integral time-varying control gain $\boldsymbol{K}_{I}(t)$. These adaptation coefficient matrices are the control 
gains tuned automatically by the adaptation mechanism in order to ensure SAC provides good tracking performance. The forgetting factors $\sigma_{e}, \sigma_{x}, \sigma_{u}$ prevent $\boldsymbol{K}_{I}(t)$ from growing too large in the presence of measurement noise (see Sec. 3.3 for more details).

The proportional-derivative controller applies the following control law:

$$
\boldsymbol{f}_{\text {control }}=\mathbf{K}_{P}\left[\mathbf{r}_{\text {des }}(t)-\mathbf{r}(t)\right]+\mathbf{K}_{D}\left[\dot{\mathbf{r}}_{\text {des }}(t)-\dot{\mathbf{r}}(t)\right]
$$

where $\mathbf{r}_{\text {des }}$ is the desired chaser position in the table frame and $\mathbf{K}_{P}$ and $\mathbf{K}_{D}$ are the proportional and derivative gain matrices, respectively (see Table 4.1 for the gain values). The adaptive control law (SAC) was previously described in Chapter 3.

\subsection{Attitude Control}

Regardless of what control law is applied for position, the attitude controller always applies the following proportional-derivative control law:

$$
\tau_{\text {control }}=K_{P, \theta}\left[\theta_{\text {des }}(t)-\theta(t)\right]+K_{D, \theta}\left[\dot{\theta}_{\text {des }}(t)-\dot{\theta}(t)\right]
$$

where $\theta_{d e s}$ is the desired angle of the chaser and $K_{P, \theta}$ and $K_{D, \theta}$ are the proportional and derivative control gains, respectively (see Table 4.1 for the gain values). The chaser controls its attitude such that its cameras are constantly pointed at the target while circling it. This work was more focused on positional error in trajectory tracking rather than attitude control.

\subsection{Perturbations}

The perturbation force present in simulation was provided by a sinusoidal function with an amplitude and bias of $0.125 \mathrm{~N}$ (so the force fluctuated between $0 \mathrm{~N}$ and $0.25 \mathrm{~N}$ ), no phase shift, and a frequency of $\frac{2 \pi}{10}$ radians per second (so each period is 10 seconds long, giving a total of 18 periods during the 180 second simulation):

$$
P=0.125 \sin \left(\frac{2 \pi}{10} t\right)+0.125
$$


This force was applied in a constant direction of 135 degrees counter-clockwise from the positive $x$ axis in the table frame, pushing the chaser towards the top right corner of the table. Note that the perturbation forces in simulation do not perfectly model those in experiments (see Sec 5.4 for more details).

\subsection{Controller Tuning}

Controller tuning began with the Proportional-Derivative (PD) controller being tuned in simulation under nominal conditions. An iterative trial-and-error process was used to tune the controller until good trajectory tracking was achieved with minimal tracking error. Then the adaptive controller was tuned in simulation under nominal conditions via an iterative trial-and-error process to match the performance of the PD controller as closely as possible. See Fig. 4.4 for the tracking errors in simulation under nominal conditions and Table 4.1 for the controller gains achieved by tuning.

The performance of both controllers needs to match under nominal conditions so that the same control gains can be used for tracking under off-nominal conditions in order to get a fair comparison of the controllers. It was difficult and tedious to tune the adaptive controller to identically match the PD controller's performance under nominal conditions, so instead the adaptive controller was tuned to perform slightly worse. This ensures that if SAC outperforms PD control under off-nominal conditions - even with a tuning disadvantage - superior performance in this application can be more confidently confirmed.

Once the final gains were achieved through tuning of the adaptive and PD controllers in simulation under nominal conditions with approximately equal performance, those same gains were used in every scenario in both experiment and simulation.

\subsection{Tracking Error}

Tracking error was measured as the absolute difference between the radius of the circular trajectory and the distance of the chaser from the center of the circular trajectory: 


$$
R_{e}(t)=\left|R_{t}-R_{c}(t)\right|
$$

where $R_{e}(t)$ is the tracking error, $R_{t}$ is the radius of the circular trajectory $(0.8 \mathrm{~m}$, see Table 4.1 for all initial conditions) and $R_{c}(t)$ is the distance of the chaser from the center of the circular trajectory, calculated using Pythagoras' theorem as follows:

$$
R_{c}(t)=\sqrt{x(t)^{2}+y(t)^{2}}
$$

where $x(t)$ and $y(t)$ are the $x$ and $y$ components of the chaser's position relative to the center of the circular trajectory.

Note that this method of calculating tracking error is unconcerned with the distance of the chaser from any given position commanded in time. Only the distance of the chaser from the circular trajectory is considered, independent of where guidance currently desires the chaser to be along the trajectory. Tracking error is determined this way for two reasons:

1. The PD controller tracks the circular trajectory directly whereas SAC feeds the trajectory through an ideal model and tracks the ideal model's output (which lags behind the trajectory according to the settling time $t_{s}$, see Table 4.1). Given that the two controllers operate in fundamentally different ways, their performance must be compared using a method that is fair and meaningful, and likely time-independent. Measuring the distance of the chaser from the circular trajectory without considering time is a straightforward and effective way of modelling tracking error that provides a fair performance comparison; no controller is unfairly favoured over another based on time-dependent differences in how they operate.

2. In the context of spacecraft proximity operations, avoiding collisions is critical. The ability of the chaser to progress along a trajectory with as little deviation as possible is generally of greater interest than the ability to complete a trajectory rapidly but inaccurately; prioritizing speed over staying on the trajectory introduces a greater risk of collision. Therefore modelling tracking error as the distance of the chaser from the circular trajectory without considering time is a meaningful result relevant to proximity operations. 
Note that the PD controller causes the chaser to progress though the trajectory more rapidly than SAC (due to the settling time of the ideal model) but both are tested for an equal amount of time; therefore the chaser actually completes slightly more of the trajectory using the PD controller. Extending the time would allow SAC to complete the trajectory.

\subsection{Contextualizing Tracking Error: Astrobee Free-flying Robots Inside the International Space Station}

In order to situate the tracking errors measured in simulations and experiments for this work into the wider context of spacecraft proximity operations, it is worthwhile to briefly discuss the Astrobee free-flying robots which NASA is planning to deploy inside the International Space Station (ISS) in 2018 [4], shown in Fig. 4.2:

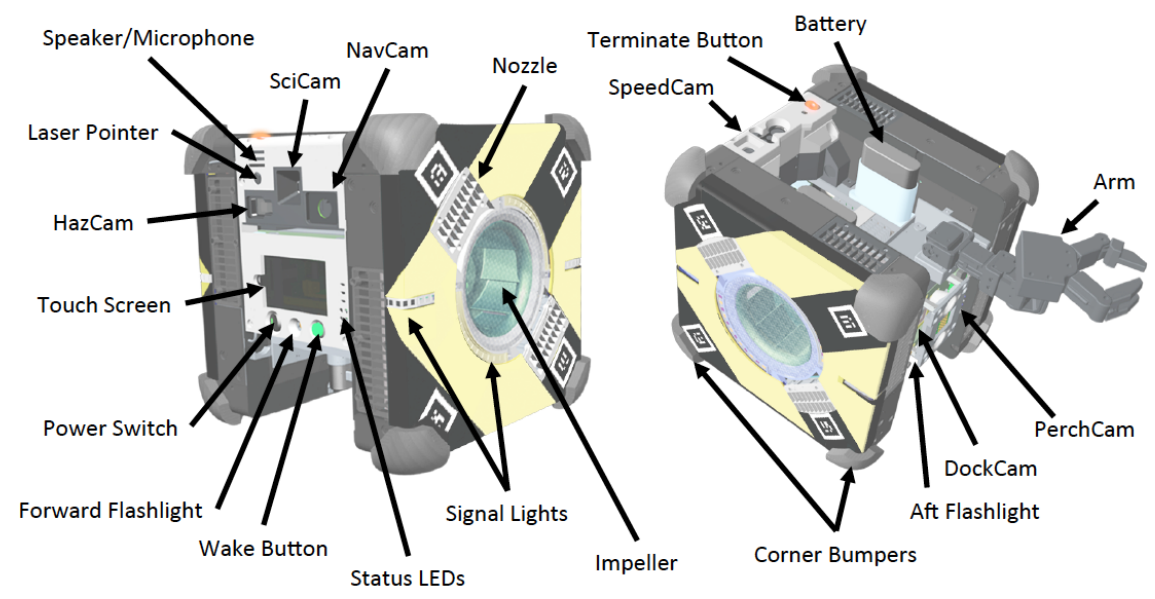

Figure 4.2: Astrobee free-flying robot $^{1}$

Astrobee free-flying robots will be able to facilitate research and experiments onboard the ISS, assist astronauts with routine chores and provide additional sensors to inspect and monitor the space station. These proximity operations inside the confines of the ISS will require careful and accurate trajectory tracking to avoid collisions and successfully capture or manipulate various objects.

Both Astrobee and the chaser platform used in this work's experiments are cube shaped with side dimensions of approximately $30 \mathrm{~cm}$ [4]. This allows the trajectory

\footnotetext{
${ }^{1}$ NASA, Astrobee (accessed September 17, 2018) https://www. nasa.gov/astrobee
} 
tracking performance measured in this work to be contextualized in a very direct manner. Astrobee and similar free-flying robotic spacecraft that perform very close proximity operations may benefit from an adaptive control strategy such as SAC to ensure good tracking performance in the presence of uncertainties and perturbations: mitigating the risk of collisions, increasing the accuracy of manipulator positioning and improving overall performance.

Note that Astrobee will be performing a similar role to SPHERES as an experimental facility for free-flying robotics onboard the ISS [4]. SPHERES also has an experimental facility on the ground (an air bearing testbed that provides a planar environment) located at the Massachusetts Institute of Technology's Space Systems Laboratory. Experimental validation of SAC at the SPHERES ground facility performed by Ulrich et al. [11] largely inspired this work; in this sense Astrobee and this work both share SPHERES as a source of inspiration.

\subsection{Simulation Results and Discussion}

A total of four different sets of conditions were simulated using both PD control and SAC as described in Sec. 4.1: nominal conditions, unknown mass, perturbations and off-nominal conditions. Note that in the results, the desired trajectory and actual position of the chaser are plotted relative to the target which is located at position $(0,0)$ in the plots. 


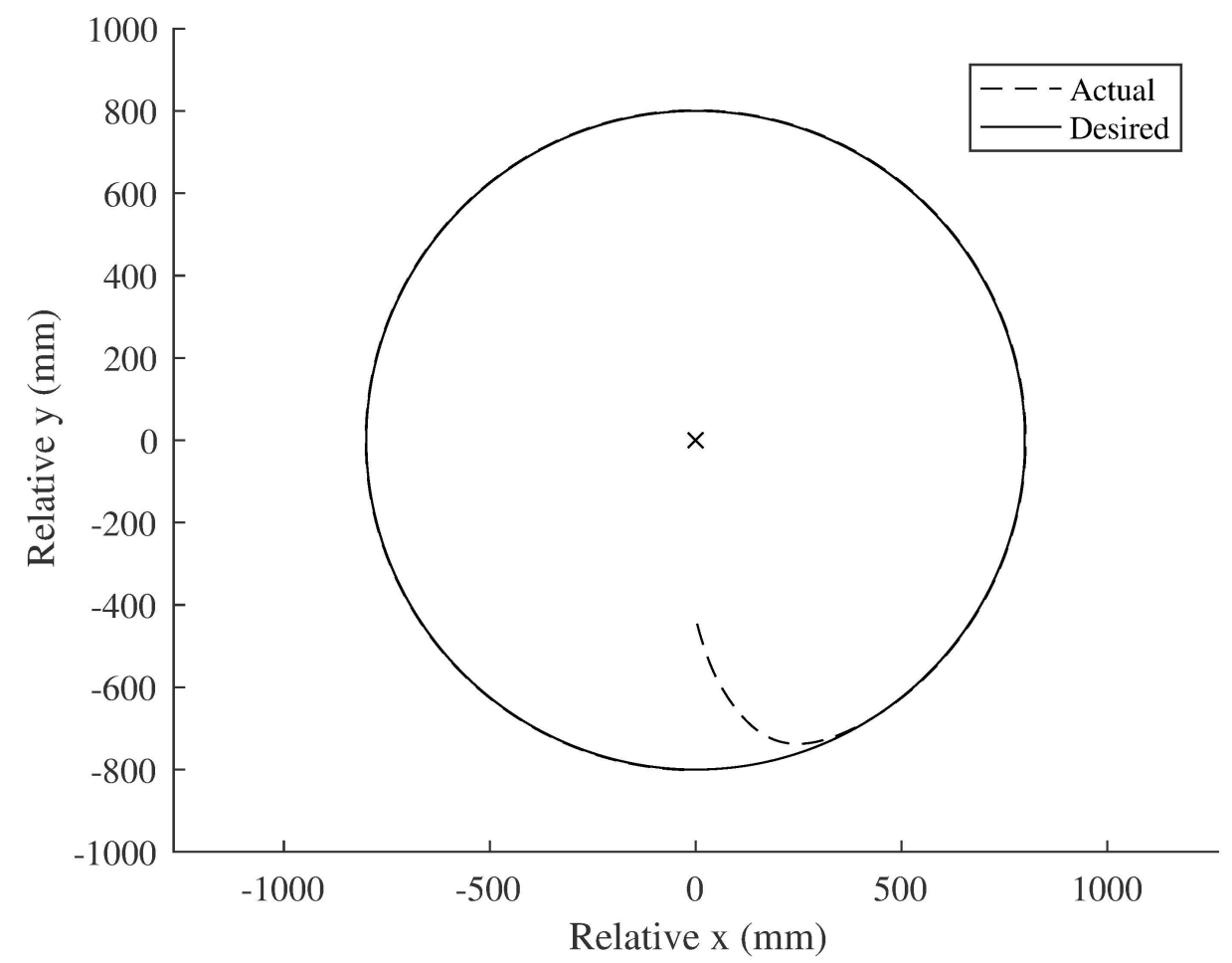

(a) PD control

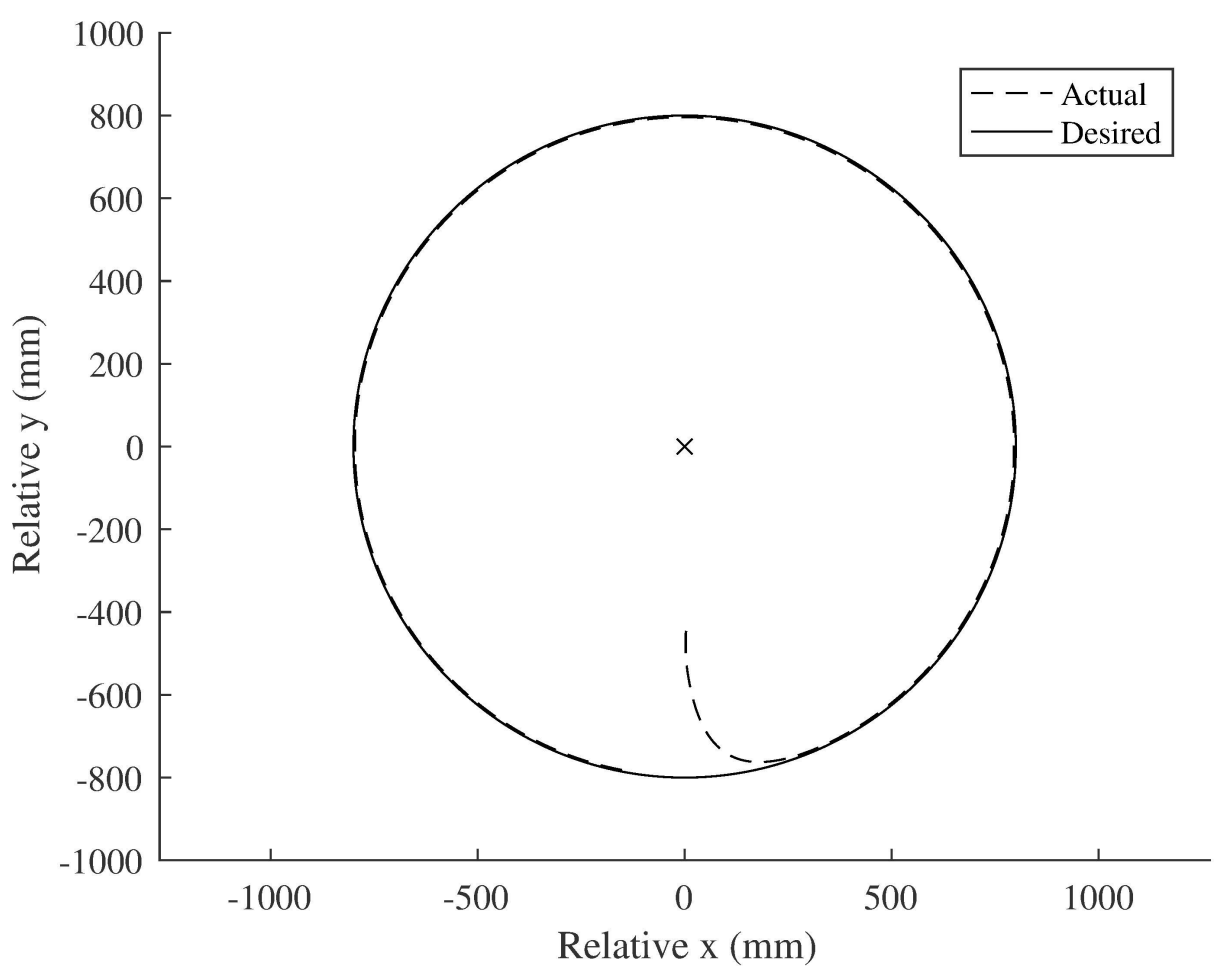

(b) $\mathrm{SAC}$

Figure 4.3: Trajectory tracking simulation under nominal conditions using (a) PD control and (b) SAC 


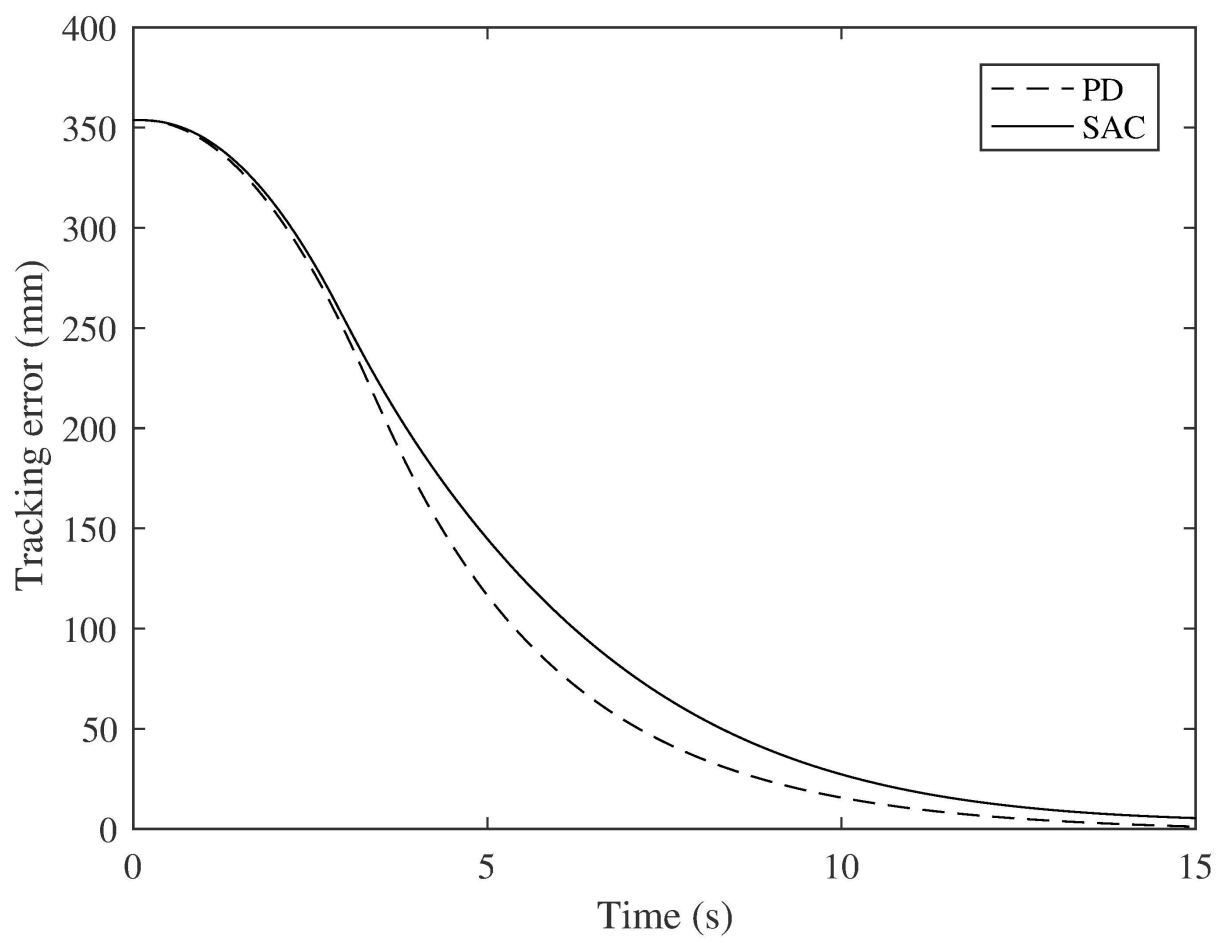

(a) Transient state (large initial error)

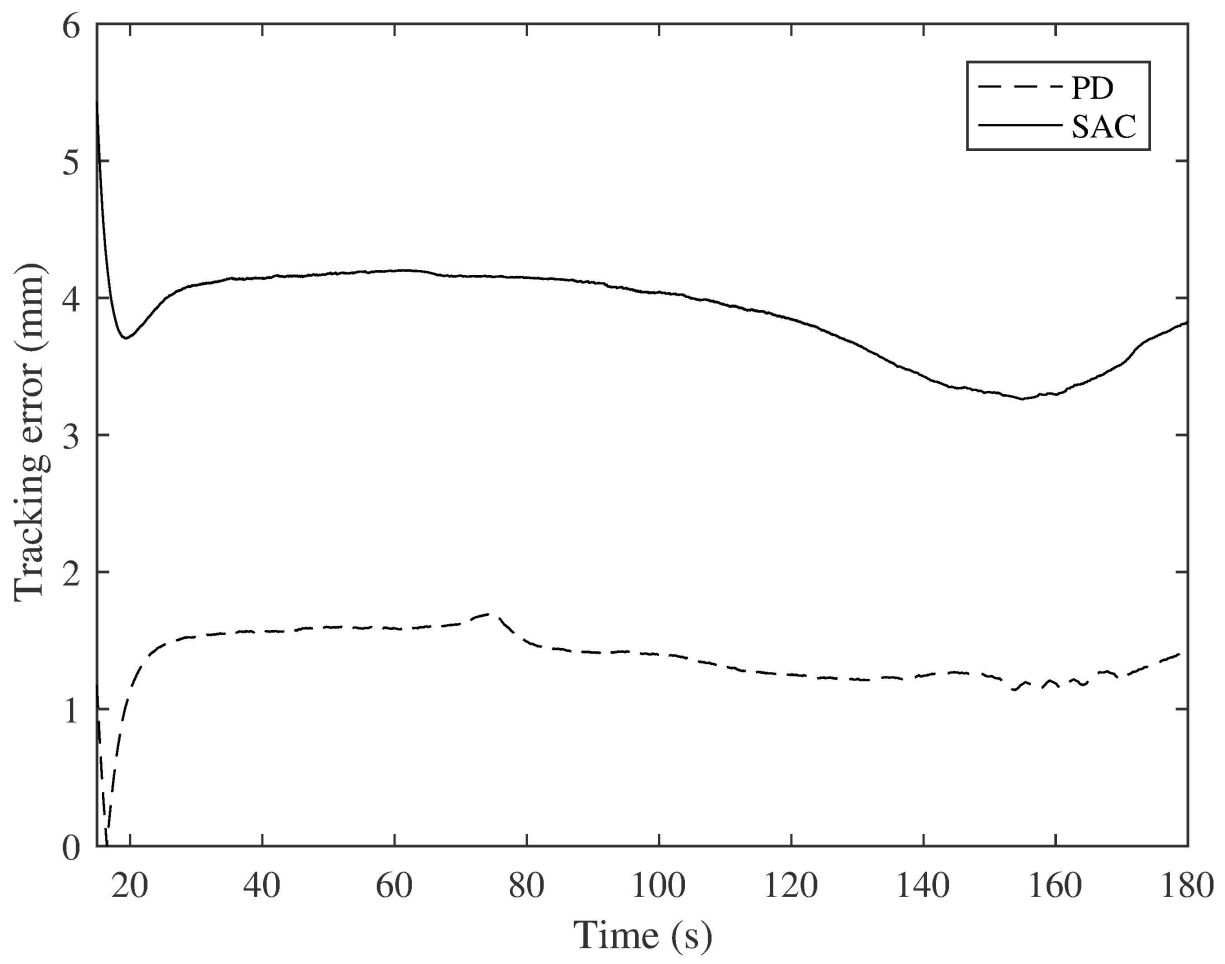

(b) Steady state

Figure 4.4: Trajectory tracking errors in simulation under nominal conditions during (a) transient state and (b) steady state 
Figs. 4.3(a) and 4.3(b) represent simulation results under nominal conditions for PD control and SAC, respectively, and Figs. 4.4(a) and 4.4(b) display the associated tracking errors for the transient and steady states, respectively.

The transient state at the beginning has a duration of approximately 15 seconds during which the large initial error in the position of the chaser is corrected as it approaches the circular trajectory. Fig. 4.4(a) displays tracking error during the transient state. There is little to note here other than SAC approaches the trajectory slightly less aggressively than the PD controller because it is tracking an ideal model output which is lagging behind the desired trajectory according to its settling time, $t_{s}$. Transient state results were included for thoroughness but will be omitted for the remaining simulation scenarios as they are repetitive and provide no further insight.

Both controllers have been successfully tuned to provide good performance under nominal conditions, as evidenced by the close match between the desired and actual trajectory in Figs. 4.3(a) and 4.3(b) for PD control and SAC, respectively. Note that as explained in Sec. 4.4, SAC was tuned to achieve slightly worse tracking than PD control because it was difficult to tune them to have identical performance and if SAC outperforms PD control under off-nominal conditions with a tuning disadvantage this demonstrates superior performance even more confidently. Fig. 4.4(b) shows that SAC is tuned to achieve a tracking error of approximately $4 \mathrm{~mm}$ whereas the PD controller is tuned to achieve a tracking error between 1 and $2 \mathrm{~mm}$, both less than $1 \%$ of the $800 \mathrm{~mm}$ radius of the commanded circular trajectory.

Now that both controllers have been tuned for satisfactory performance under nominal conditions, the controller gains (listed in Table 4.1) will no longer be changed, and remain constant in all other scenarios as the system is subjected to perturbation forces and unknown changes in mass. This is important for keeping the mass change unknown to the system because retuning the gains to accommodate a mass change would effectively mean that the system knows the mass, which would violate the intended conditions of the scenario (unknown mass change).

The following scenario investigates the effect of adding an unknown mass to the system. 


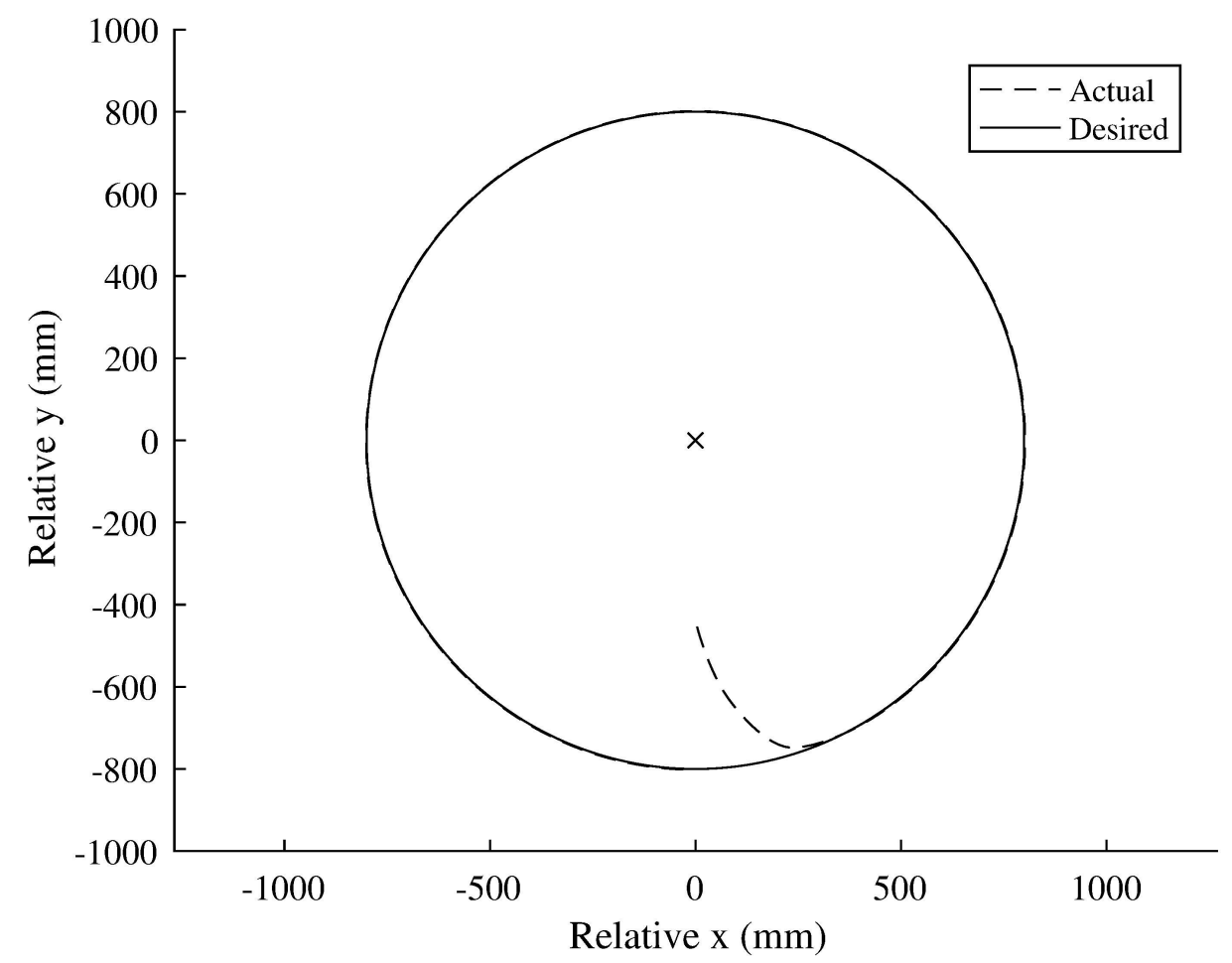

(a) PD control

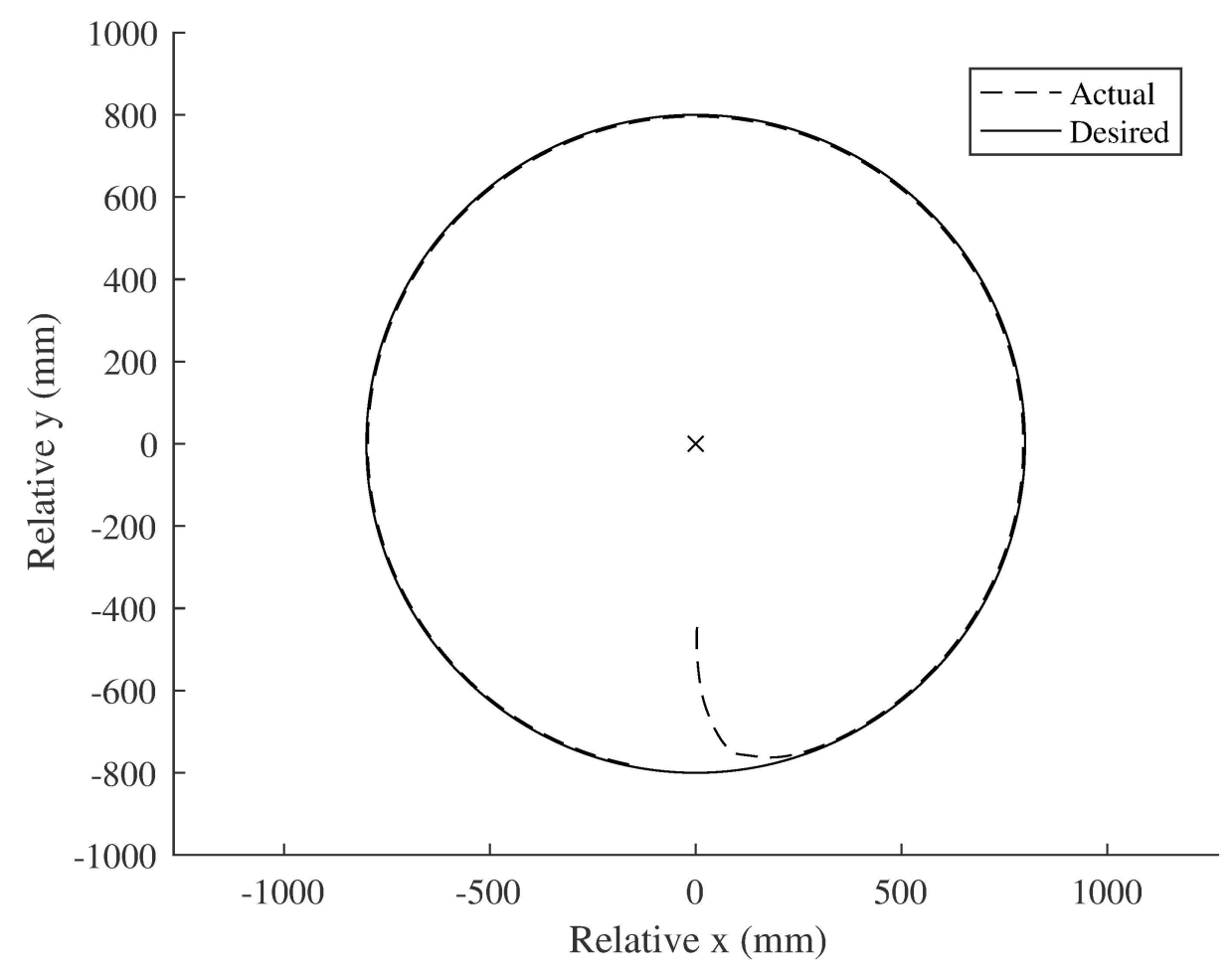

(b) $\mathrm{SAC}$

Figure 4.5: Trajectory tracking simulation with an unknown mass added to the system using (a) PD control and (b) SAC 


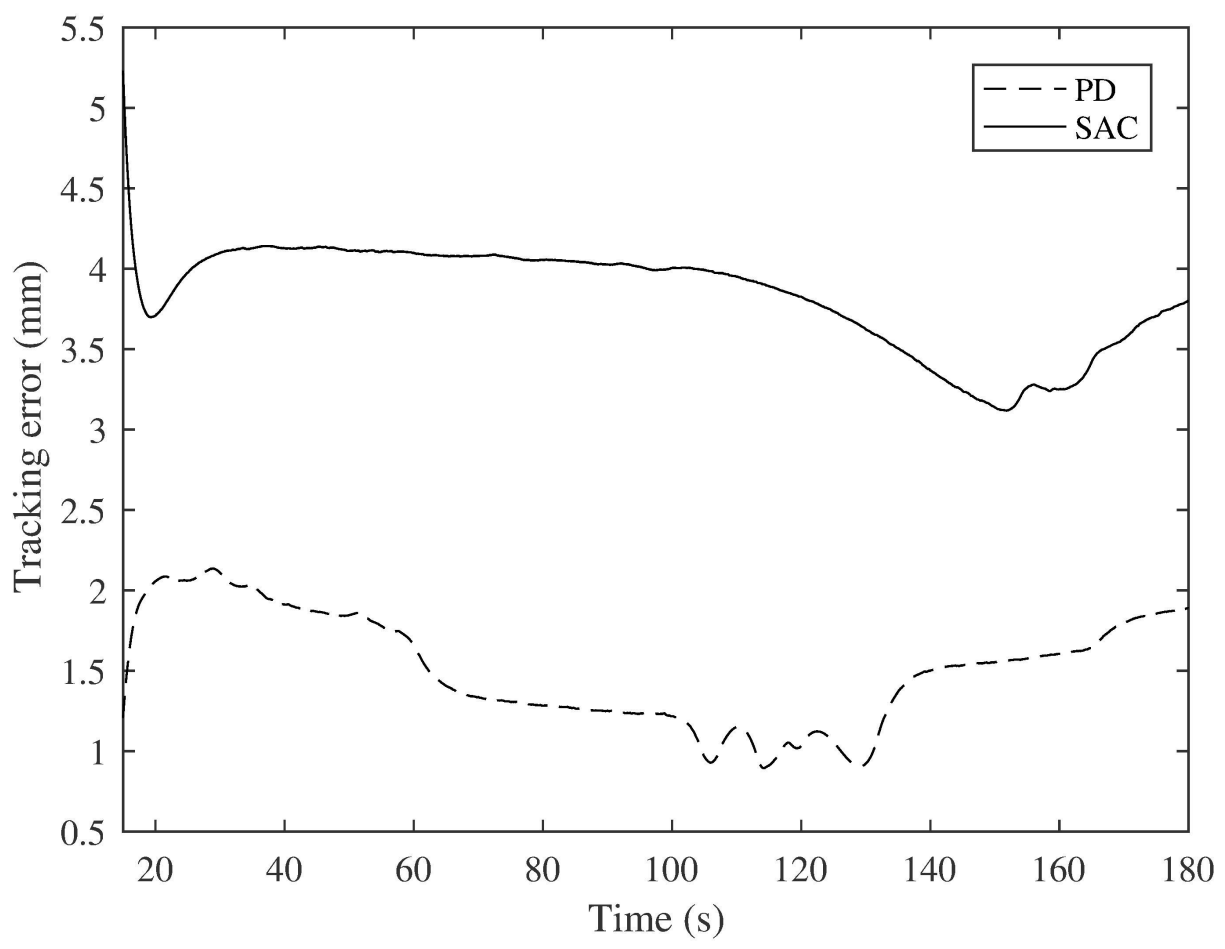

Figure 4.6: Trajectory tracking errors in simulation with an unknown mass added to the system

Figs. 4.5(a) and 4.5(b) represent simulation results with an unknown mass added to the system for PD control and SAC, respectively, and Fig. 4.6 displays the associated tracking errors. Note that the mass was increased from $m_{\text {nom }}=17.24 \mathrm{~kg}$ to $m_{\text {off }}=$ $26.37 \mathrm{~kg}$ (unknown to the system) representing a substantial increase of approximately $50 \%$. In application this represents a scenario where the chaser spacecraft has a poor estimate of its mass, for example after capturing debris of uncertain mass, but still has tracking requirements.

The addition of an unknown mass to the system had little impact on the match between the desired and actual trajectory in Figs. 4.5(a) and 4.5(b) for PD control and SAC, respectively. Looking more closely at the tracking errors in Fig. 4.6 and comparing them to the nominal case, it is notable that the PD controller performs slightly worse with the addition of an unknown mass to the system, exceeding $2 \mathrm{~mm}$ of tracking error at the beginning and experiencing larger fluctuations in tracking error as compared to nominal conditions. This is to be expected as the PD controller is tuned for the nominal mass $m_{n o m}$, not the new unknown mass of the system $m_{\text {off }}$. 
In contrast, SAC exhibited virtually no change in performance and the tracking error remained approximately $4 \mathrm{~mm}$ as the controller adapted to the mass change.

The following scenario investigates the effect of applying perturbation forces to the system without changing its mass. This allows the two effects of perturbation forces and unknown mass changes to be compared in isolation before combining them in the final scenario. 


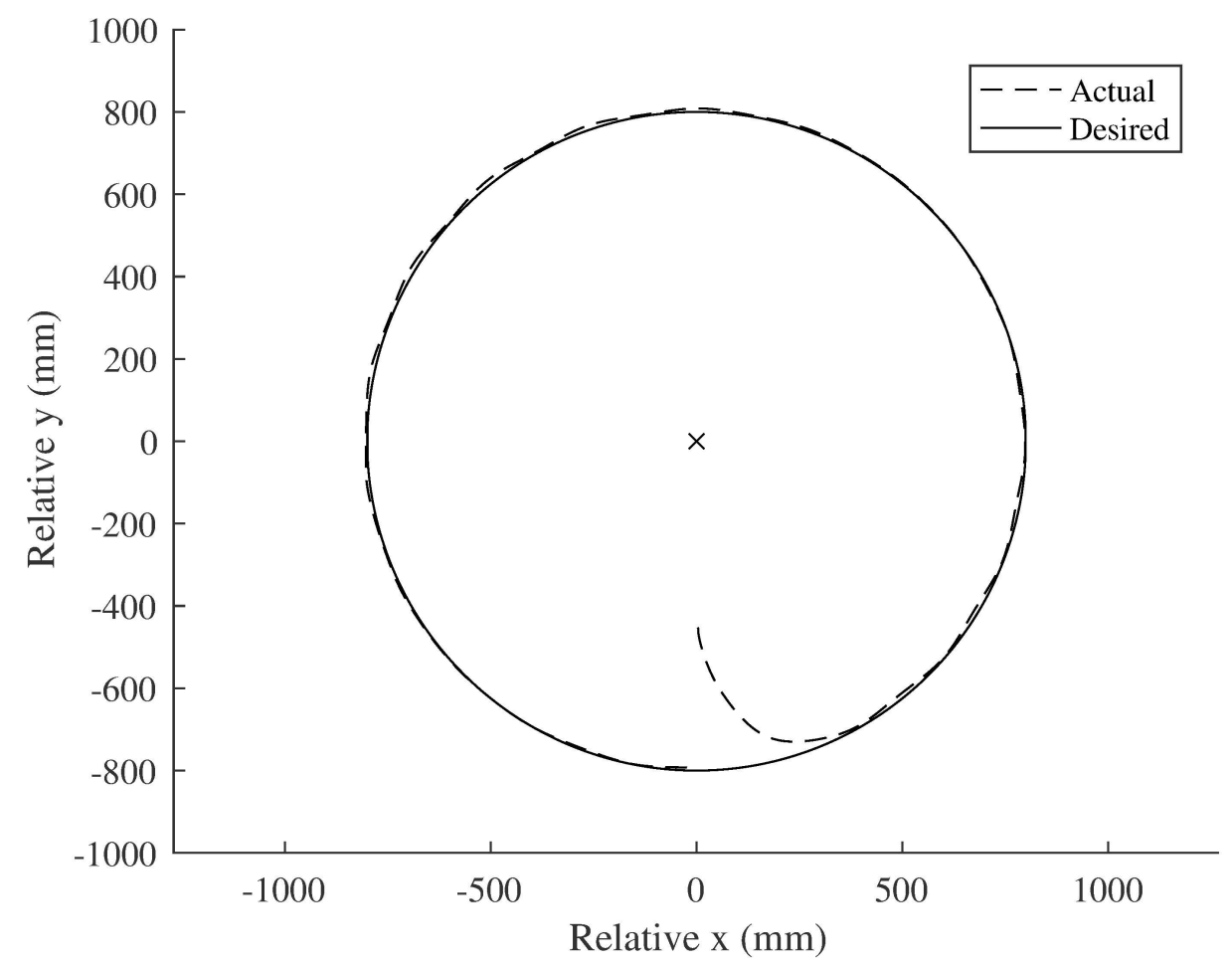

(a) PD control

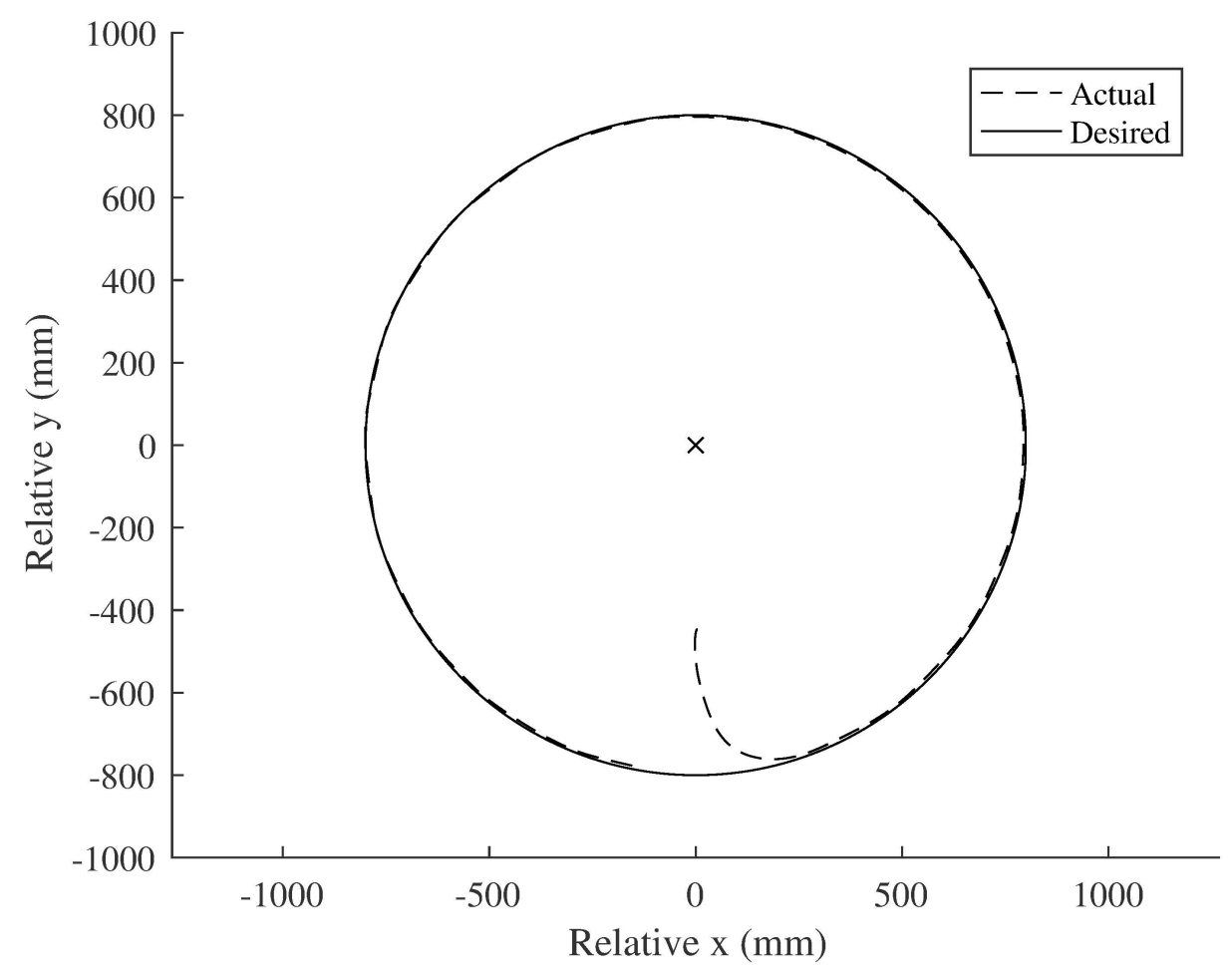

(b) $\mathrm{SAC}$

Figure 4.7: Trajectory tracking simulation with perturbations using (a) PD control and (b) SAC 


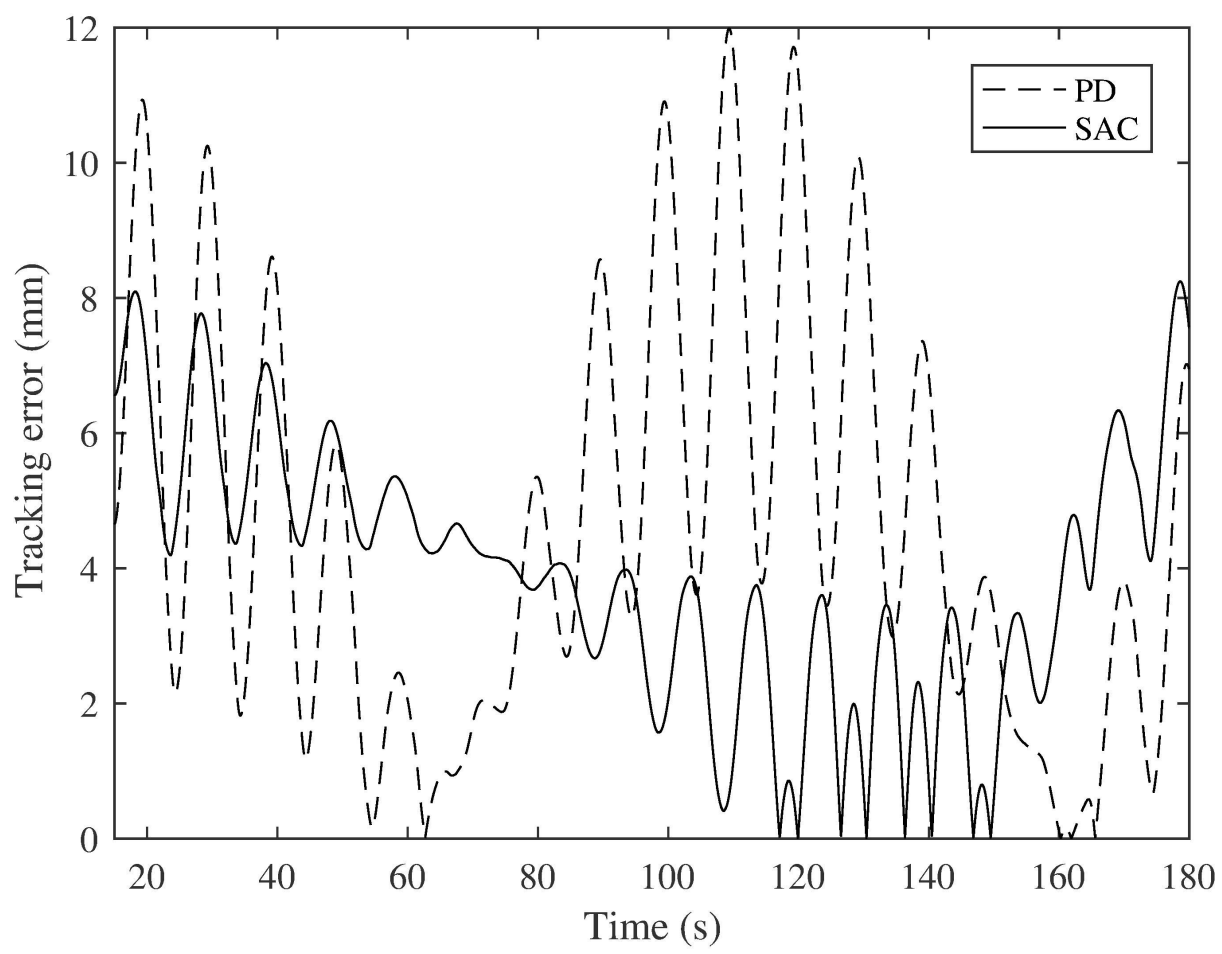

Figure 4.8: Trajectory tracking errors in simulation with perturbations

Figs. 4.7(a) and 4.7(b) represent simulation results with perturbations for PD control and SAC, respectively, and Fig. 4.8 displays the associated tracking errors.

Applying perturbations forces to the system in simulation (described in Sec. 4.3) had a visually detectable impact on the match between the desired and actual trajectory in Figs. 4.7(a) and 4.7(b) for PD control and SAC, respectively, as compared to the nominal and unknown mass change scenarios. Using PD control, the chaser's actual trajectory is beginning to deviate from the desired trajectory enough that the two are separated by a noticeable gap, whereas they are still partially or entirely overlapping for SAC. Looking more closely at the tracking errors in Fig. 4.8 and comparing them to the unknown mass change scenario, it is clear that the perturbation forces had a more significant impact on tracking error which is fluctuating as high as $8 \mathrm{~mm}$ for SAC and $12 \mathrm{~mm}$ for PD control. Despite starting with a performance disadvantage under nominal conditions as compared to PD control, SAC is now outperforming PD control when perturbation forces are applied to the system. The ability of SAC to adapt to changing conditions has allowed it to keep tracking error generally lower and with smaller fluctuations. 
Note how the tracking error profile for both controllers contains both high frequency and low frequency variations. The high frequency variations are largely caused by the sinusoidal nature of the perturbation force magnitude in simulation. The low frequency variations are caused by the changing angle between the perturbation force vector and the chaser's velocity vector. The perturbation force is applied in a constant direction of 135 degrees counter-clockwise from the positive $x$ axis in the table frame, pushing the chaser towards the top right corner of the table (see Sec. 4.3) whereas the chaser's velocity is constantly changing direction to remain tangent to the circular trajectory during tracking. When the perturbation forces are parallel or antiparallel to the chaser's velocity they either assist or hinder its progress along the trajectory, respectively, but produce minimal tracking error because they do not change the direction of the chaser's velocity (corresponding to the troughs in low frequency variations of tracking error). When the perturbation forces are perpendicular to the chaser's velocity they produce maximum tracking error as they cause the largest change in the chaser's direction, steering it off the trajectory (corresponding to the peaks in low frequency variations of tracking error).

High and low frequency variations in tracking error are more clearly pronounced in the case of PD control, with an especially notable low frequency variation peak around $110 \mathrm{~s}$ where tracking error reaches $12 \mathrm{~mm}$. This is triple the corresponding tracking error of SAC which appears to be steadily reducing tracking error due to its adaptive nature, partially eliminating the low frequency variations that PD control is experiencing.

Now that the effects of unknown mass changes and perturbation forces in simulation have been investigated separately, the final scenario combines these two effects to investigate how SAC and PD control perform under fully off-nominal conditions that are more representative of a real application in debris removal and spacecraft proximity operations. 


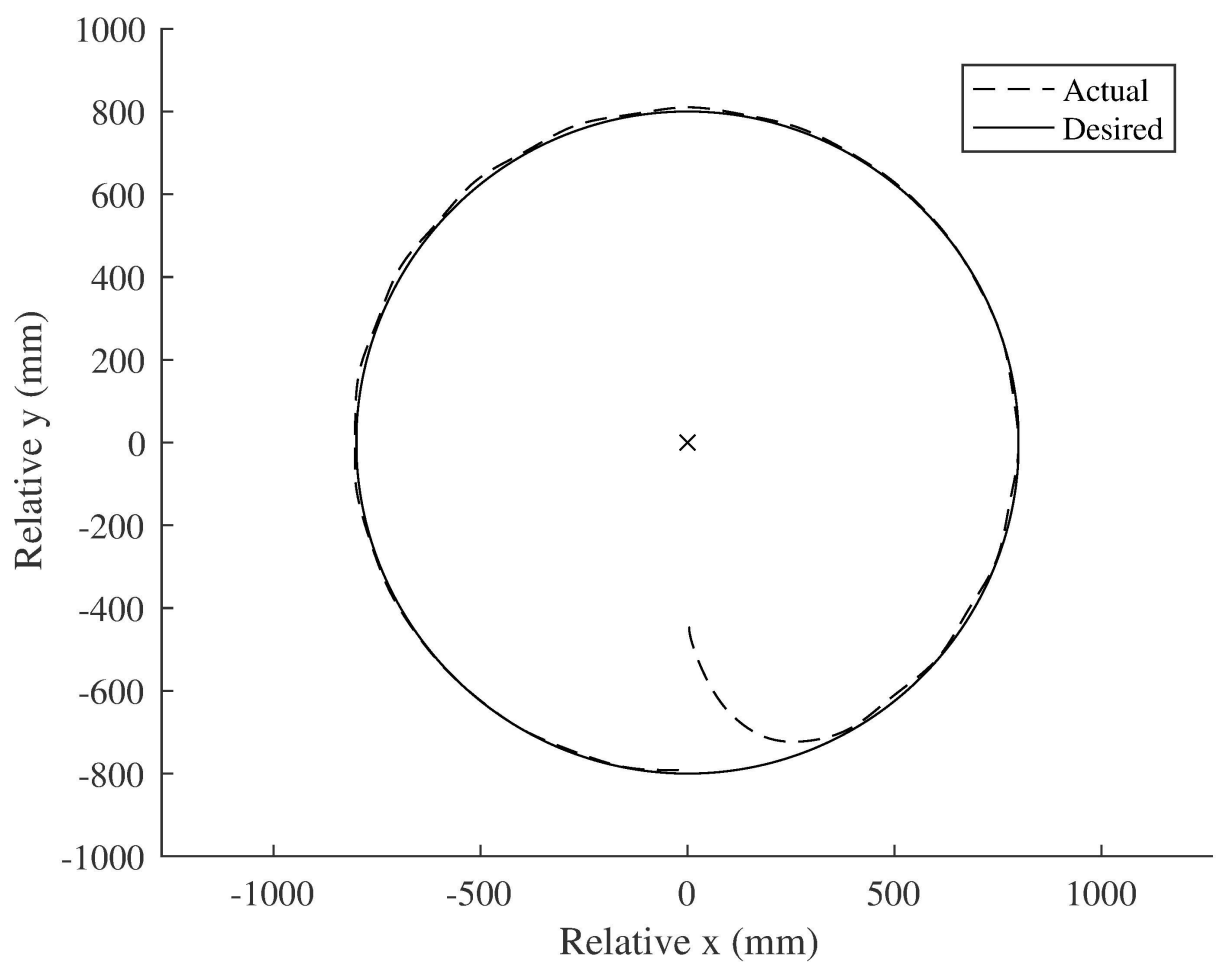

(a) PD control

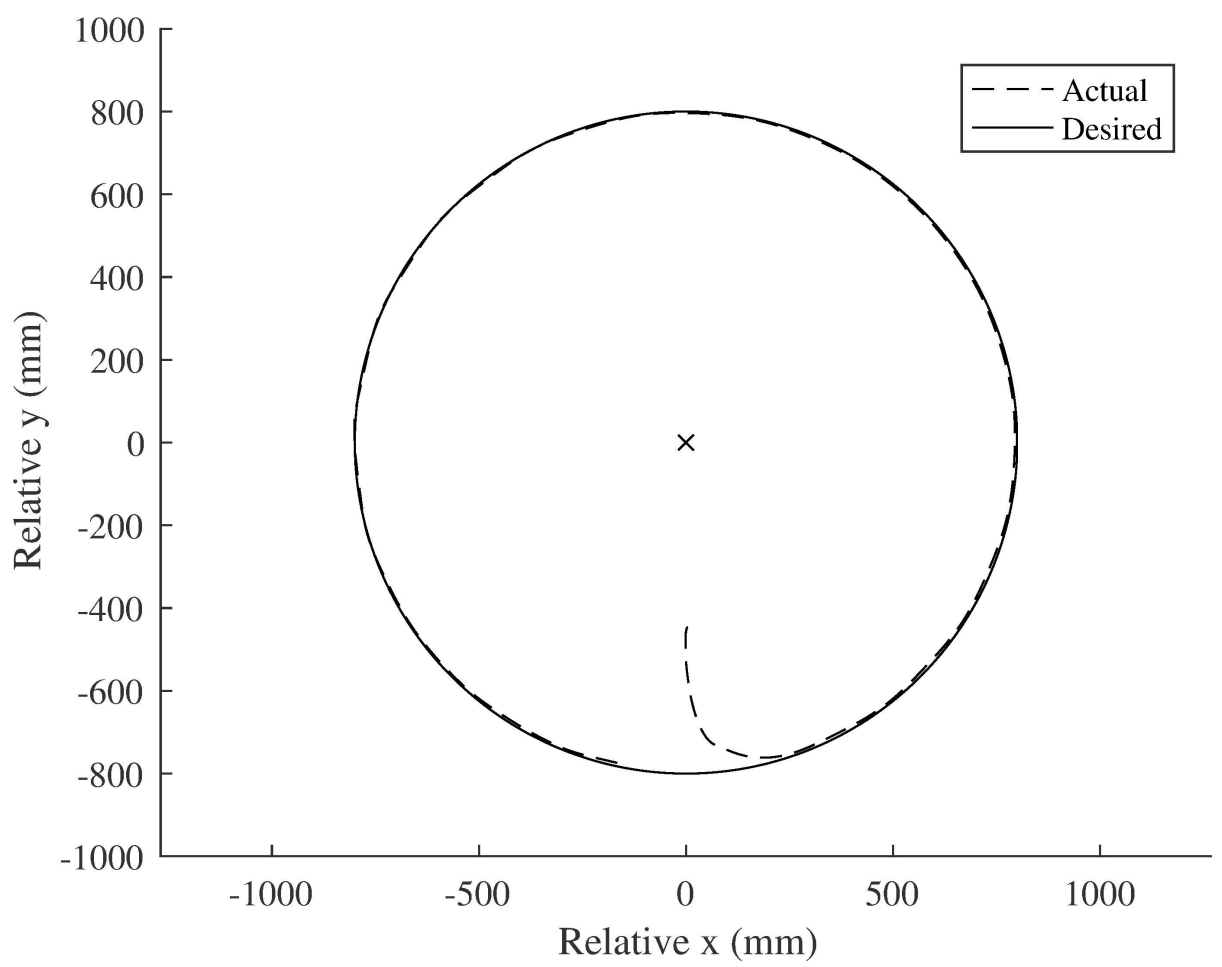

(b) SAC

Figure 4.9: Trajectory tracking simulation under off-nominal conditions using (a) PD control and (b) SAC 


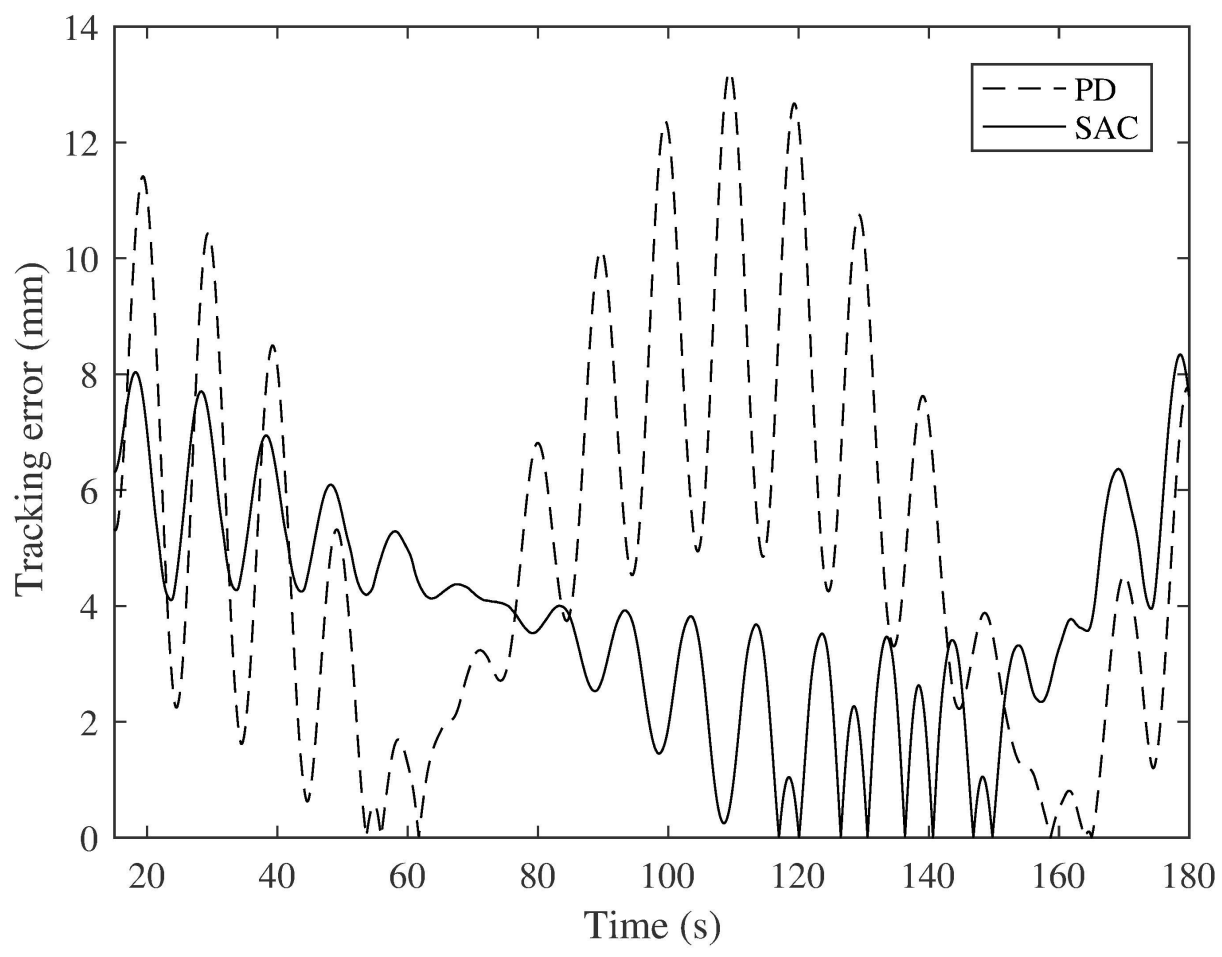

Figure 4.10: Trajectory tracking errors in simulation under off-nominal conditions

Figs. 4.9(a) and 4.9(b) represent simulation results under off-nominal conditions for PD control and SAC, respectively, and Fig. 4.10 displays the associated tracking errors. The chaser had its mass increased by approximately $50 \%$ from $m_{n o m}=17.24$ $\mathrm{kg}$ to $m_{\text {off }}=26.37 \mathrm{~kg}$ without being informed of this change or having its controller gains retuned (to represent capturing space debris of unknown mass) and it is being subjected to perturbation forces as described in Sec. 4.3.

The tracking error profile for both controllers in Fig. 4.10 is very similar to Fig. 4.8 where only perturbations were present. Perturbations are clearly the dominant effect on tracking error in off-nominal conditions, however the mass increase combined with perturbations has slightly increased tracking error for PD control above either the perturbations or mass increase alone. Under fully off-nominal conditions, PD control reached a peak tracking error of approximately $13 \mathrm{~mm}$, an increase from $12 \mathrm{~mm}$ when only perturbations were present. This increase in peak tracking error is approximately equal to the one found when comparing nominal conditions to the addition of an unknown mass without perturbations.

The same high frequency and low frequency variations in tracking error are present 
due to perturbations, with their magnitude slightly increased by the addition of an unknown mass for PD control. SAC appears to have been unaffected by the mass increase in fully off-nominal conditions, with its tracking error profile in Fig. 4.10 nearly identical to the scenario with only perturbations in Fig. 4.8.

\subsection{Conclusion}

There are two important conclusions from the simulation results:

1. SAC outperformed PD control in all scenarios beyond nominal conditions despite worse tuning of its control gains, further reinforcing the superiority of its performance.

2. Perturbations influence tracking error more significantly than adding an unknown mass to the system, however the presence of both effects has a larger influence than either one in isolation.

Now that all four scenarios have been successfully tested in simulation, the next step is to validate these results by performing experiments. 


\section{Chapter 5}

\section{Experiments}

This chapter introduces the Spacecraft Proximity Operations Testbed (SPOT) in the Spacecraft Robotics and Control Laboratory at Carleton University, describes the author's contributions in designing and building this facility, and presents experimental results obtained from this facility in which a chaser platform tracks a circular trajectory around a target on an air bearing testbed to validate the numerical simulations performed in Chapter 4.

\subsection{Experimental Facility}

Experiments were conducted at Carleton University's Spacecraft Robotics and Control Laboratory, using the Spacecraft Proximity Operations Testbed (SPOT). Specifically, the SPOT consists of two air-bearing spacecraft platforms operating in close proximity on a $2.4 \times 3.7 \mathrm{~m}$ granite surface. The use of air bearings on the platforms reduces the friction to a negligible level. Due to surface slope, residual gravitational accelerations perturb the dynamics of the floating platforms (see Table 4.1). Both platforms have dimensions of $0.3 \times 0.3 \times 0.3 \mathrm{~m}$ and are actuated by expelling compressed air at $550 \mathrm{kPa}$ through eight miniature air nozzles distributed around each platform, thereby providing full planar control authority. Each thruster generates approximately $0.25 \mathrm{~N}$ of thrust and is controlled at a frequency of $500 \mathrm{~Hz}$ by a pulsewidth modulation scheme using solenoid valves. Pressurized air for the thrusters and the air-bearing flotation system is stored on board in a single air cylinder at $31 \mathrm{MPa}$. The structure consists of an aluminum frame with four corner rods, on which three modular decks are stacked. To protect the internal components, the structure is covered with semitransparent acrylic panels. Fig. 5.1 shows a fully assembled SPOT spacecraft platform: 


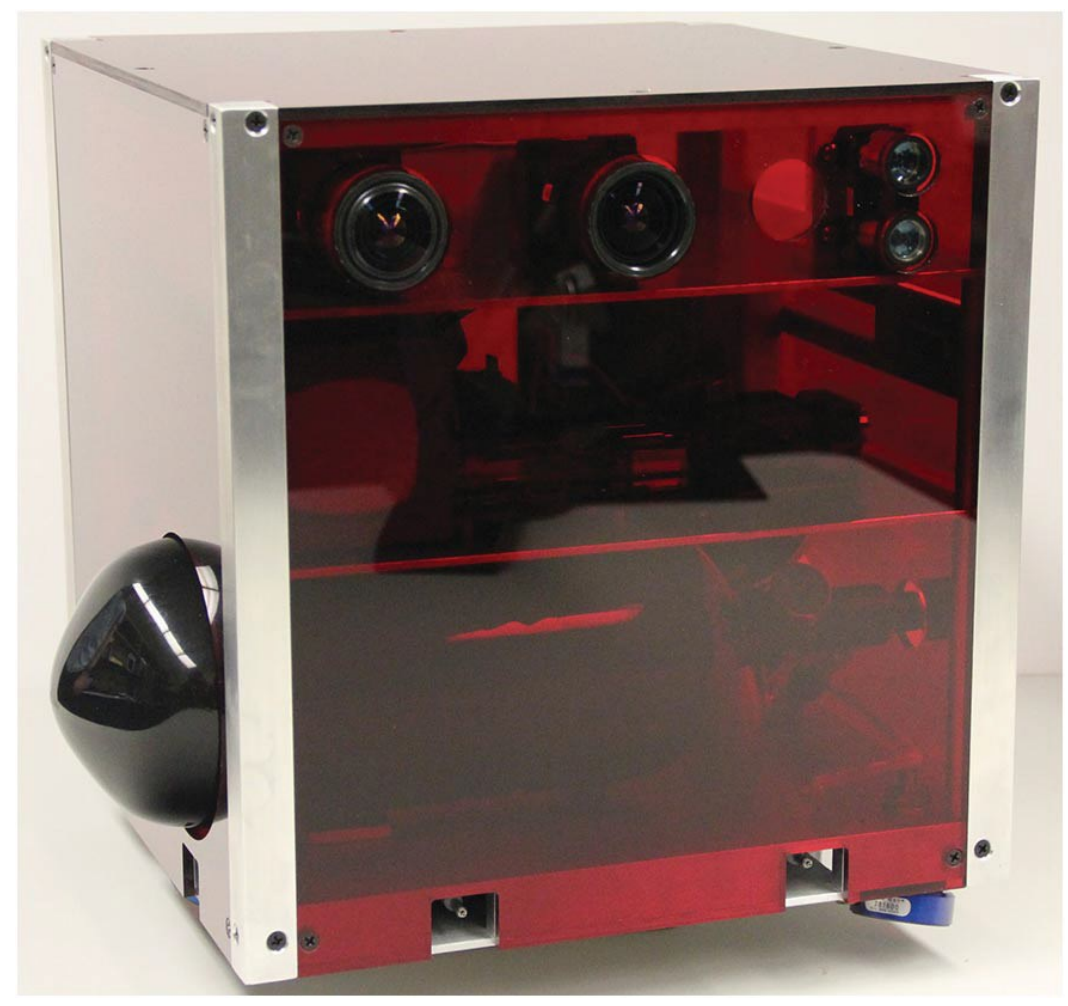

Figure 5.1: SPOT platform built at Carleton University

The motion of both platforms is measured in real time through four active lightemitting diodes (LEDs) on each platform, which are tracked by an eight-camera PhaseSpace(C) motion-capture system. This provides highly accurate ground-truth position and attitude data. All motion-capture cameras are connected to a PhaseSpace server, which is connected to a ground station computer. The ground station computer communicates, via a wireless user datagram protocol, the ground-truth information to the platforms' onboard computers, which consist of Raspberry Pi-3s running the Raspbian Linux operating system. Based on the position and attitude data that the platforms wirelessly receive, they can perform feedback control by calculating the required thrust to autonomously maneuver, as well as actuating the appropriate solenoid valves to realize this motion. The ground station computer also receives real-time telemetry data (i.e., any signals of interest, as specified by the user) from all onboard computers for post-experiment analysis purposes. A MATLAB/Simulink numerical simulator that recreates the dynamics and emulates the different onboard sensors and actuators is first used to design and test the upcoming experiment. Once 
the performance in simulations is satisfactory, the control software is converted into $\mathrm{C} / \mathrm{C}++$ using Embedded Coder@, compiled, and then executed on the platforms' Raspberry Pi-3 computers. An overview of the SPOT can be seen in Fig. 5.2:

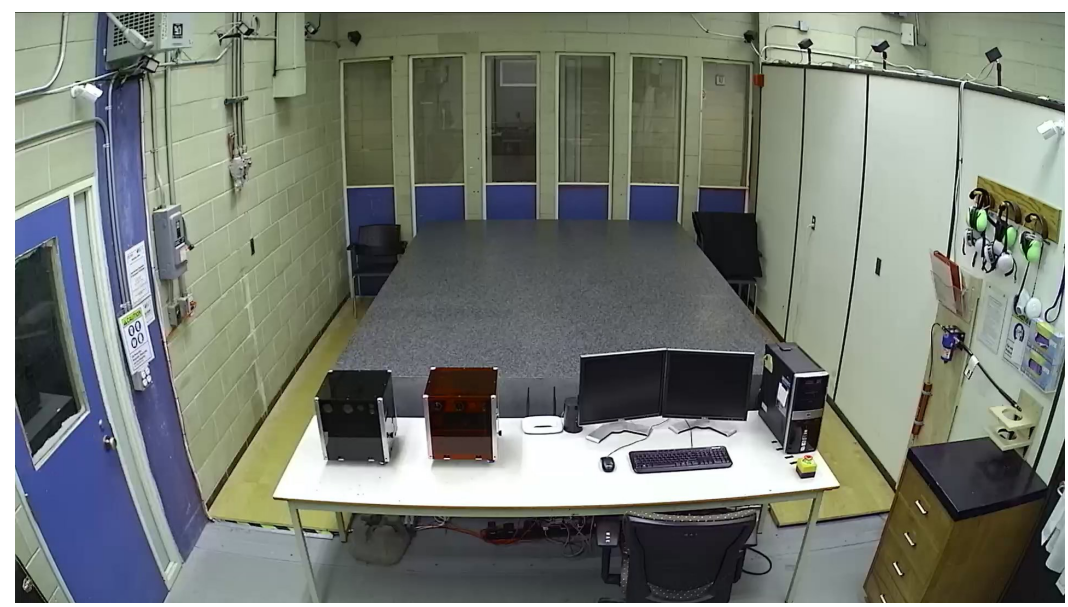

Figure 5.2: Overview of the Spacecraft Proximity Operations Testbed

\subsection{Lab Contributions}

The author was responsible for creating the software environment that enabled simulations and experiments and interfacing the hardware and software systems in real-time. Fig. 5.3 provides a general overview of the software environment.

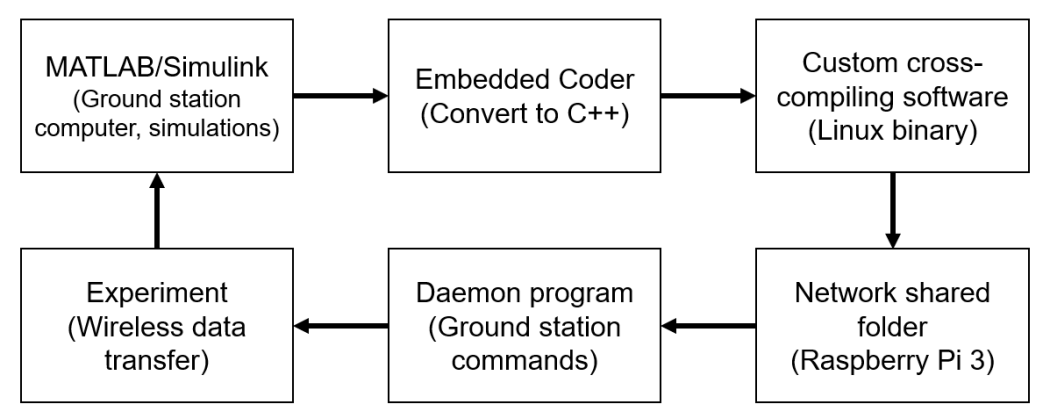

Figure 5.3: Software Environment

A single MATLAB/Simulink diagram was created to perform both simulations and experiments which is summarized in Fig. 5.4: 


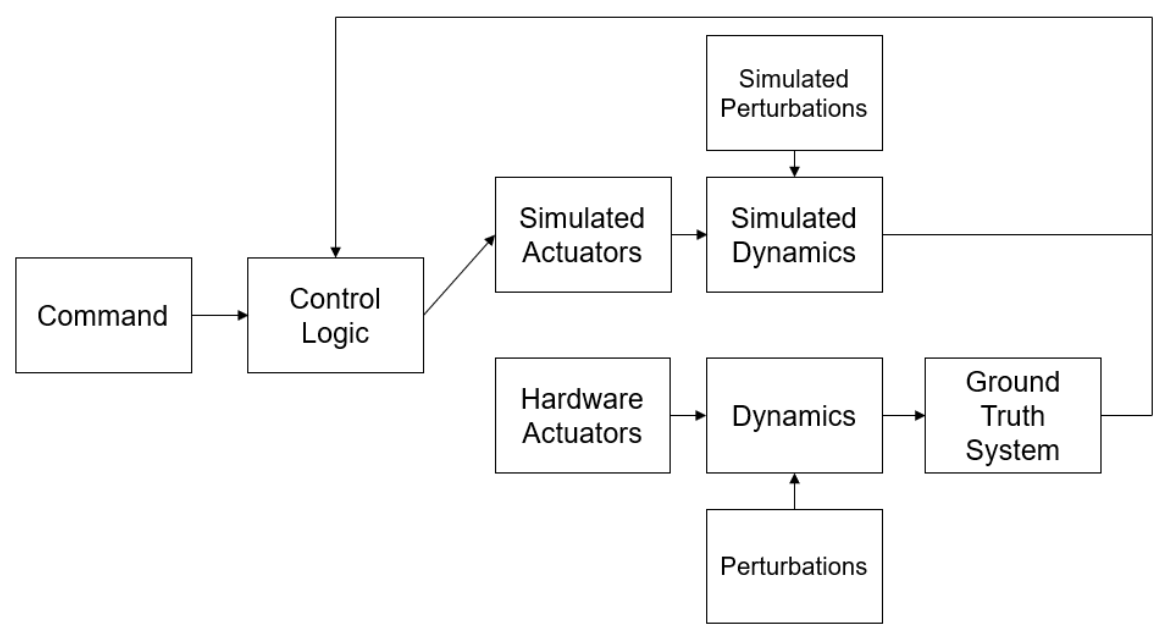

(a) Simulation configuration

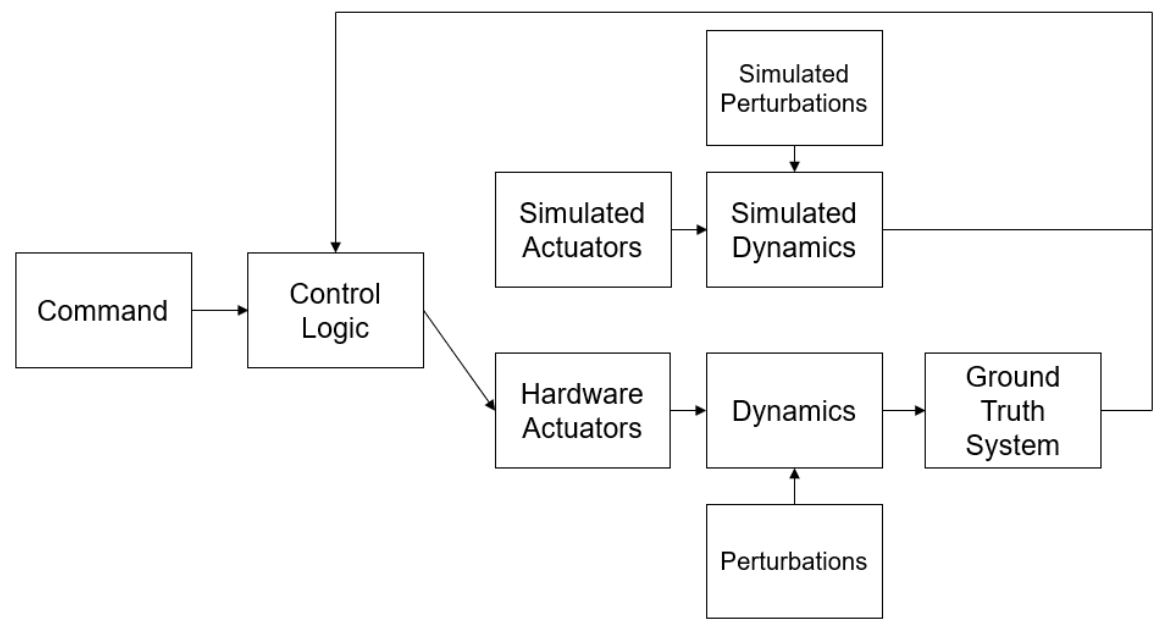

(b) Experiment configuration

Figure 5.4: Overview of the MATLAB/Simulink diagram used in the experimental facility configured for (a) simulations and (b) experiments

Note that both simulations and experiments were performed using the diagram in Fig. 5.4 where a logic switch enables the user to specify which configuration to use (Fig. 5.4(a) for simulations and Fig. 5.4(b) for experiments). This means that the ability to perform simulations is built into and not separate from the process used for experiments and the logic is identical (with the exception of the simulated dynamics and perturbations). 
The diagram summarized in Fig. 5.4 included S-function blocks which acted as wrappers to interface with sensors and actuators for experiments. The wrappers were written in $\mathrm{C}++$ and called hardware-specific library functions. Simulations were performed in MATLAB/Simulink on the ground station computer. In order to perform experiments, the diagram was converted into $\mathrm{C}++$ code using Embedded Coder in MATLAB/Simulink. A custom program was written in $\mathrm{C}++$ for the purpose of automatically cross-compiling the Embedded Coder output into a Linux binary. This binary was immediately accessible to the onboard processor (Raspberry Pi 3) of the chaser platform through a shared network folder. A daemon program was written in $\mathrm{C}++$ to allow the chaser platform to be controlled from the ground station computer in order to run binaries and begin experiments. A wireless communication program was written in $\mathrm{C}++$ in order to send experimental data from the chaser platform to the ground station computer.

This software environment enabled any given scenario to be simulated using a MATLAB/Simulink diagram and then rapidly exported for performing experiments. The exporting process was largely automatic, required no modifications to the MATLAB/Simulink diagram and took less than one minute.

\subsection{Experimental Setup}

The experiments performed were identical to the simulations presented in Chapter 4 with the exception of the perturbation forces as described in Sec. 5.4. The experimental setup is described by the diagram in Fig. 5.5: 

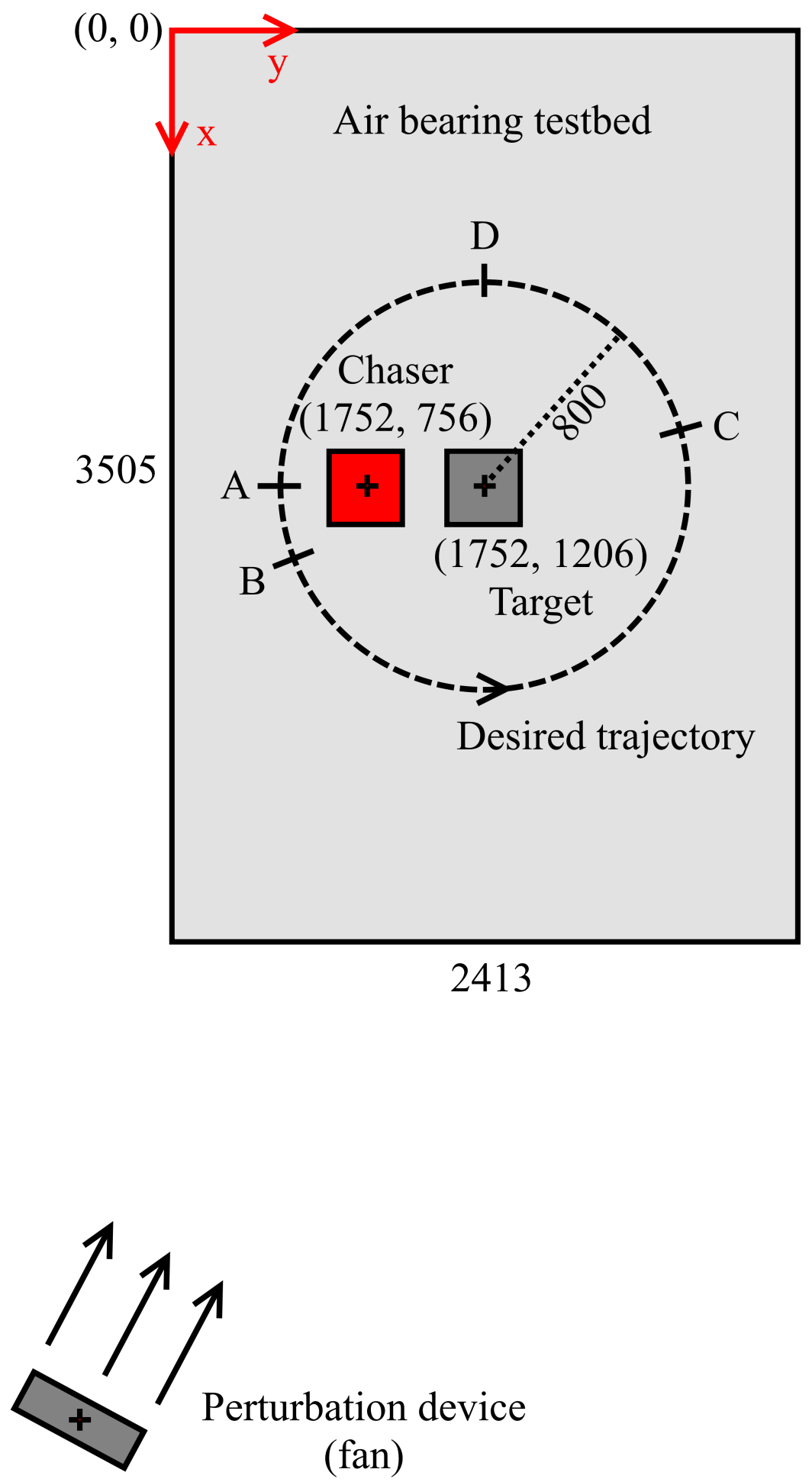

$(5436,-343)$

Figure 5.5: Experimental setup (all dimensions in $\mathrm{mm}$ ) 
where points A, B, C and D refer to points where changes occur in the operation of the perturbation device during the experiment (see Sec. 5.4). The chaser and target platforms are represented by the red and black bodies in the diagram, respectively. The reference frame represented in the diagram is the table frame and all positions are given in this frame. The desired trajectory occurs in a counter-clockwise direction as indicated. The perturbation device (fan blowing air on the chaser platform) is located at a distance from the table. Note that the target platform is included in the diagram to represent the debris that the chaser would be circling in a real mission however the target platform is not actually included in the experiment. The target platform was placed on the table once during an experiment for the purposes of recording a video (no data was collected from it, it was only a visual aid) as in Fig. 5.6:

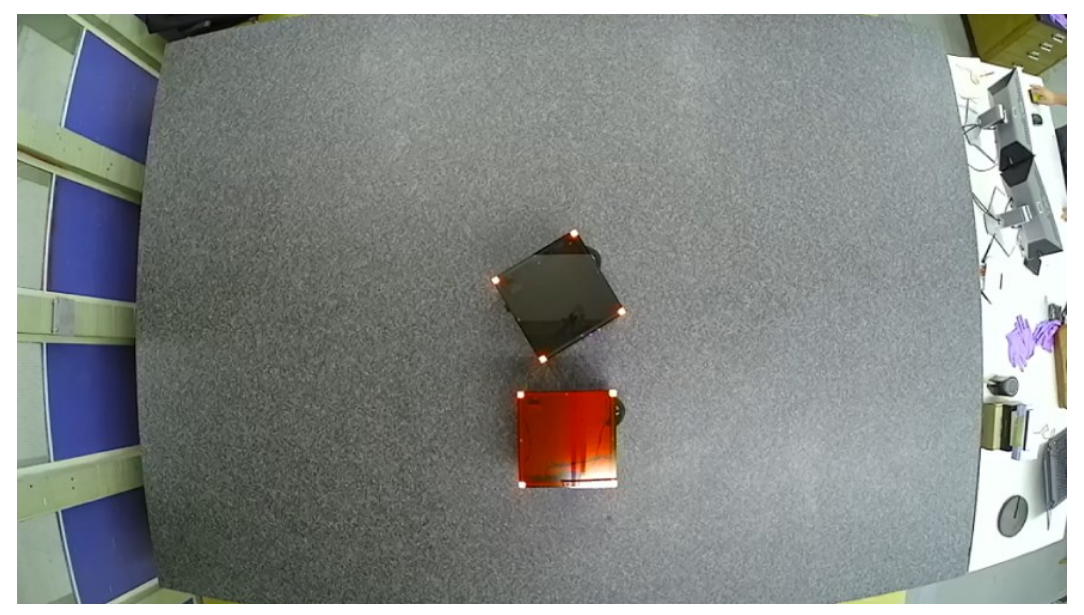

Figure 5.6: Experiment initial conditions

\subsection{Perturbations}

The perturbation forces present in experiment were produced by the perturbation device seen in Fig. 5.7. It was mounted on a rotating platform with a laser pointer to indicate the direction of perturbation forces. 


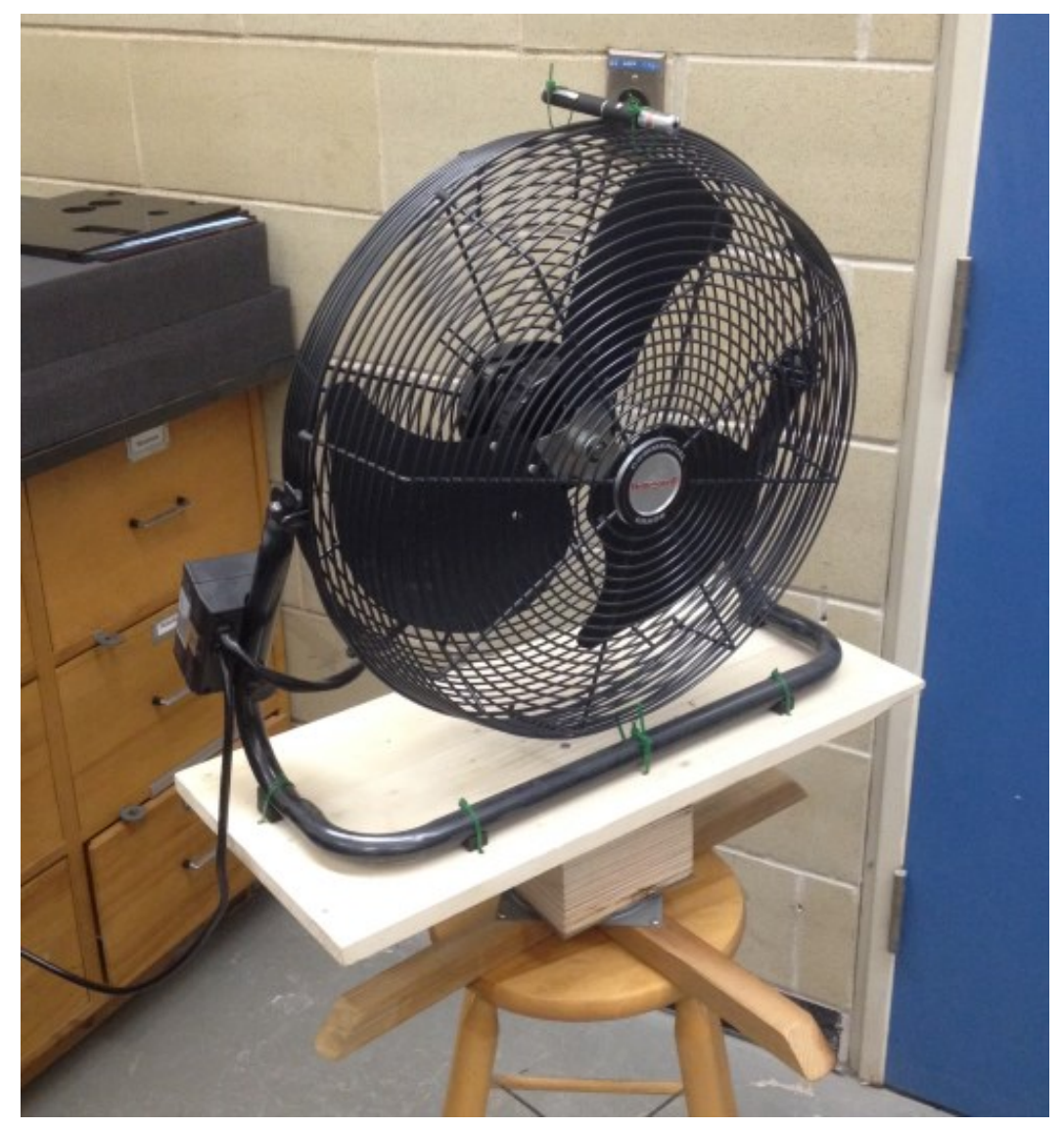

Figure 5.7: Perturbation device: Honeywell HV180 fan mounted on rotating platform with laser pointer

The center of the fan was located at $(5.436 \mathrm{~m},-0.343 \mathrm{~m})$ in the table frame as depicted in Fig. 5.5 and the base of the fan was located $0.813 \mathrm{~m}$ above the floor (the air bearing testbed surface was located $0.927 \mathrm{~m}$ above the floor). During experiments where perturbations were present, an operator stood behind the fan to manually control the direction and magnitude of perturbation forces. The following procedure was executed by the operator where points A, B, C and D are points along the trajectory as defined in Fig. 5.5:

1. The experiment begins with the chaser inside the commanded circular trajectory near point $\mathrm{A}$. The operator does not yet turn on the fan but keeps it pointed directly at the center of the chaser at all times, verifying its direction with the laser pointer. 2. The chaser reaches the trajectory at point B (approximately 15 seconds) and the operator turns on the fan to the 'LOW' speed setting. 
3. The chaser reaches point $\mathrm{C}$ and the operator changes the fan speed setting to 'MEDIUM'.

4. The chaser reaches point $\mathrm{D}$ and the operator changes the fan speed setting to 'LOW'.

The fan is kept off for approximately 15 seconds at the beginning of the experiment to allow the chaser to reach the trajectory. The fan speed is varied inversely with the chaser's distance from the fan to maintain an appropriate perturbation force magnitude.

The author acknowledges that, though similar in magnitude and direction, the perturbation forces present in simulation (see Sec. 4.3) were not identical to those in experiments. Perfect modelling of perturbations in simulation to match experiments would have been challenging but also unnecessary as the purpose of this work is to compare the performance of PD control against SAC for trajectory tracking, not to compare simulations against experiments.

The important difference between perturbations in simulation and experiment is that the simulation perturbations are perfectly sinusoidal and periodically decrease to a magnitude of zero, whereas the experiment perturbations provided by the fan fluctuate less predictably and likely never decrease to zero as the fan is constantly aimed at the chaser and not turned off until the end of the experiment. The attitude of the chaser also has an influence on the magnitude of perturbations in experiment due to the surface area exposed to the fan. When the cube-shaped chaser is oriented such that its side is facing the fan, the surface area upon which the fan can apply air pressure is only $30 \mathrm{~cm}$ by $30 \mathrm{~cm}$. When the chaser is oriented such that its corner is facing the fan, two sides are exposed and the surface area upon which the fan can push includes the diagonal length of the chaser which is approximately $42 \mathrm{~cm}$. This represents an increase in surface area of approximately $40 \%$ which for a given air pressure also corresponds to an increase in perturbation force magnitude of $40 \%$. The perturbations in experiment present a more significant challenge to the controller and therefore are of greater interest for trajectory tracking and practical application.

In practical application the perturbation forces produced by the fan could represent typical perturbations that a spacecraft would experience in orbit such as J2 
perturbations, atmospheric drag, solar radiation pressure and third body perturbations. The air pressure produced by the fan is most analogous to solar radiation pressure as these effects both involve surface area and pressure to generate forces on the spacecraft and are not retarding forces like drag. The effect of perturbation forces on tracking performance could also provide insight into the ability of the chaser to withstand perturbing forces associated with capture maneuvers and the operation of robotic arms.

\subsection{Statistical Analysis}

In each experimental scenario a total of five trials were performed in order to generate data for statistical analysis. The following equation was used to calculate three standard deviations of dispersion from the mean of a desired quantity:

$$
\text { Dispersion }=\bar{x} \pm 3 \sigma
$$

where $\bar{x}$ is the mean of the data and $\sigma$ is the standard deviation:

$$
\sigma=\sqrt{\frac{\sum_{n=1}^{N}\left(x_{n}-\bar{x}\right)^{2}}{N-1}}
$$

where $N$ is the sample size $\left(N=5\right.$ for five trials) and $x_{n}$ is the sample from the $n$th trial. The ability to repeat an experiment and have a desired quantity (such as chaser position) change over time in a predictable and precise manner, with as little dispersion in the data between trials as possible, is of interest for good trajectory tracking performance.

\subsection{Experimental Results and Discussion}

A total of four different sets of conditions were tested in experiment using both PD control and SAC: nominal conditions, unknown mass, perturbations and off-nominal conditions. Note that in the results, the desired trajectory and actual position of the chaser are plotted relative to the target which is located at position $(0,0)$ in the plots. 


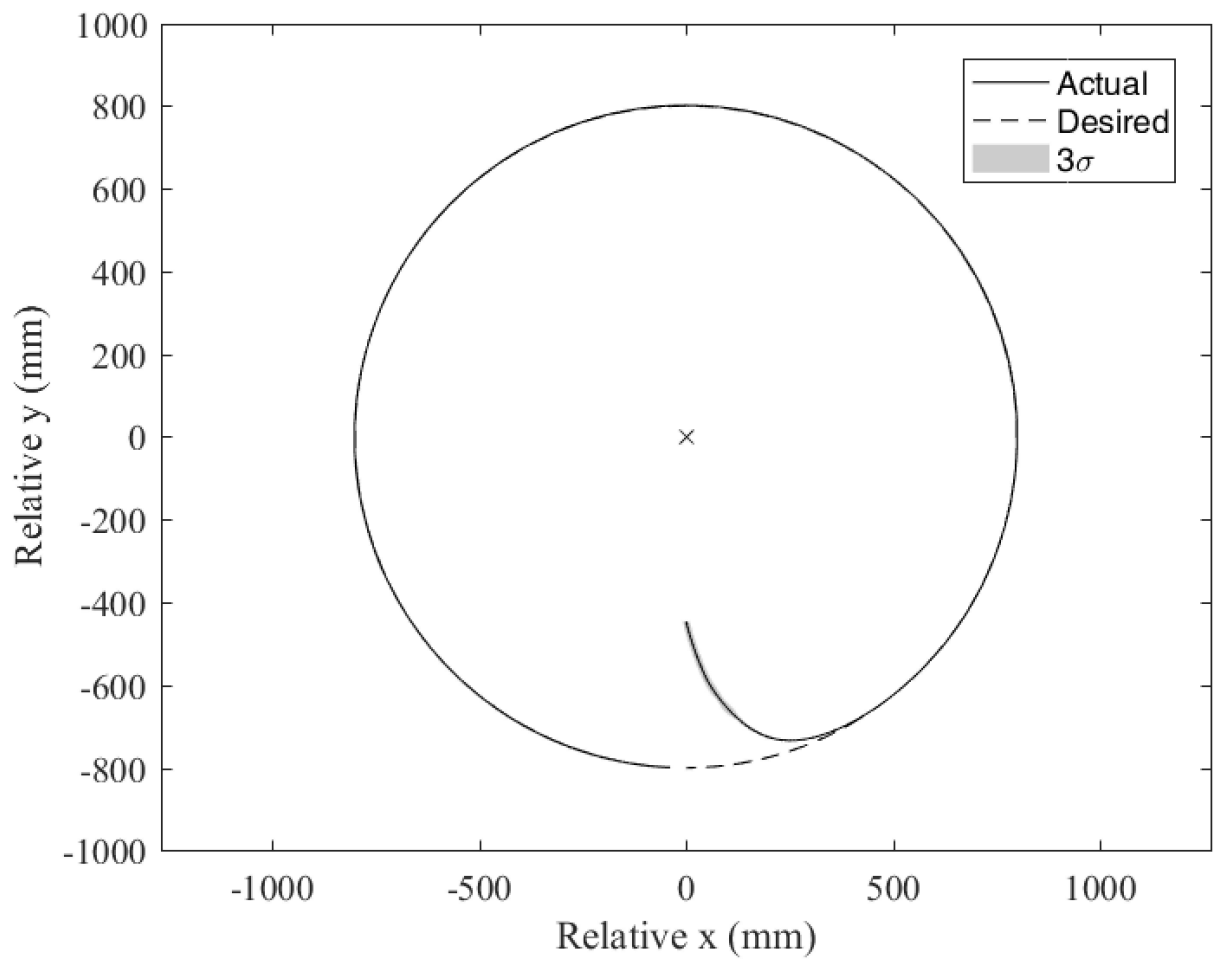

(a) PD control

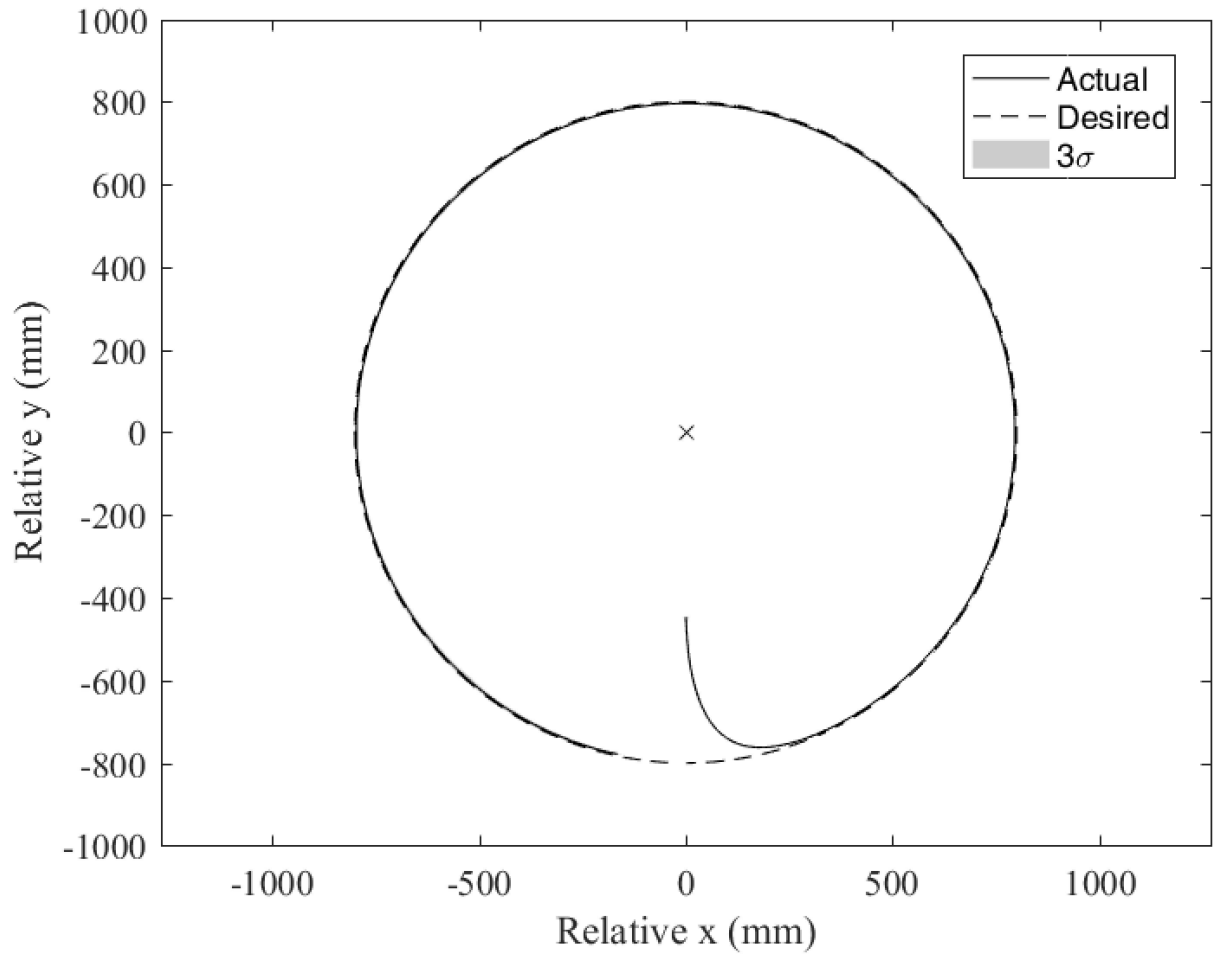

(b) SAC

Figure 5.8: Trajectory tracking experiment under nominal conditions using (a) PD control and (b) SAC 


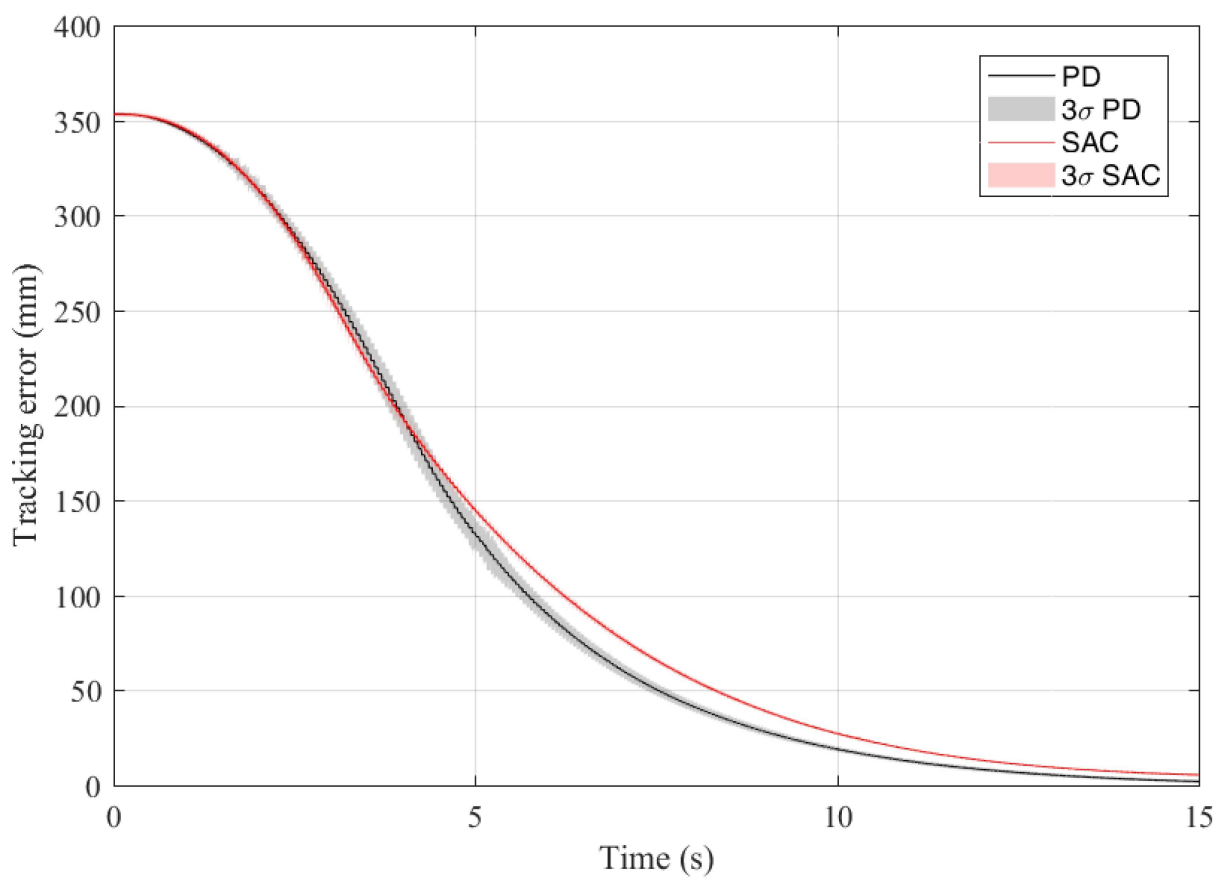

(a) Transient state (large initial error)

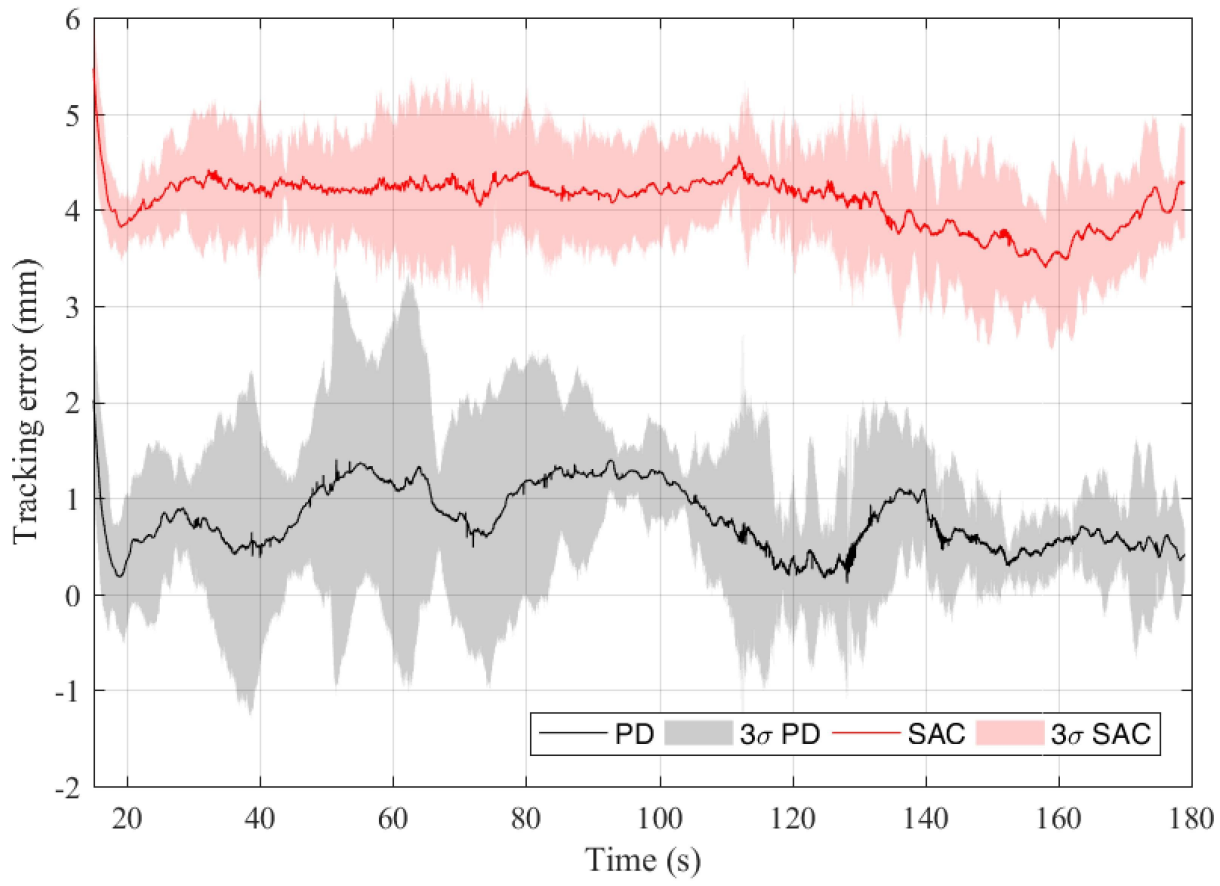

(b) Steady state

Figure 5.9: Trajectory tracking errors in experiment under nominal conditions during (a) transient state and (b) steady state

Figs. 5.8(a) and 5.8(b) represent experimental results under nominal conditions for 
PD control and SAC, respectively, and Figs. 5.9(a) and 5.9(b) display the associated tracking errors for the transient and steady states, respectively.

Similarly to the results presented for simulations, the transient state at the beginning has a duration of approximately 15 seconds during which the large initial error in the position of the chaser is corrected as it approaches the circular trajectory. Fig. 5.9(a) displays tracking error during the transient state. Note how the tracking error data for PD control is already exhibiting greater $3 \sigma$ dispersion than SAC even under nominal conditions, indicating worse precision and repeatability between experiments. SAC approaches the trajectory slightly less aggressively than the PD controller because it is tracking an ideal model output which is lagging behind the desired trajectory according to its settling time, $t_{s}$. Transient state results were included for thoroughness but will be omitted for the remaining experimental scenarios as they are repetitive and provide no further insight.

The gains tuned in simulation (listed in Table 4.1) are successfully providing good tracking performance in experiments under nominal conditions, as evidenced by the close match between the desired and actual trajectory in Figs. 5.8(a) and 5.8(b) for PD control and SAC, respectively. Note that as explained in Sec. 4.4, SAC was tuned to achieve slightly worse tracking than PD control because it was difficult to tune them to have identical performance and if SAC outperforms PD control under off-nominal conditions with a tuning disadvantage then this demonstrates superior performance even more confidently. Fig. 5.9(b) shows that SAC and PD control are tuned to achieve tracking errors of approximately $4 \mathrm{~mm}$ and $1 \mathrm{~mm}$, respectively, less than $1 \%$ of the $800 \mathrm{~mm}$ radius of the commanded circular trajectory and closely matching simulation results for nominal conditions.

Recall that the controller gains (listed in Table 4.1) have been tuned for satisfactory performance under nominal conditions and will remain constant in all other scenarios in order to provide a fair comparison between SAC and PD control.

The following experimental scenario investigates the effect of adding an unknown mass to the system. 


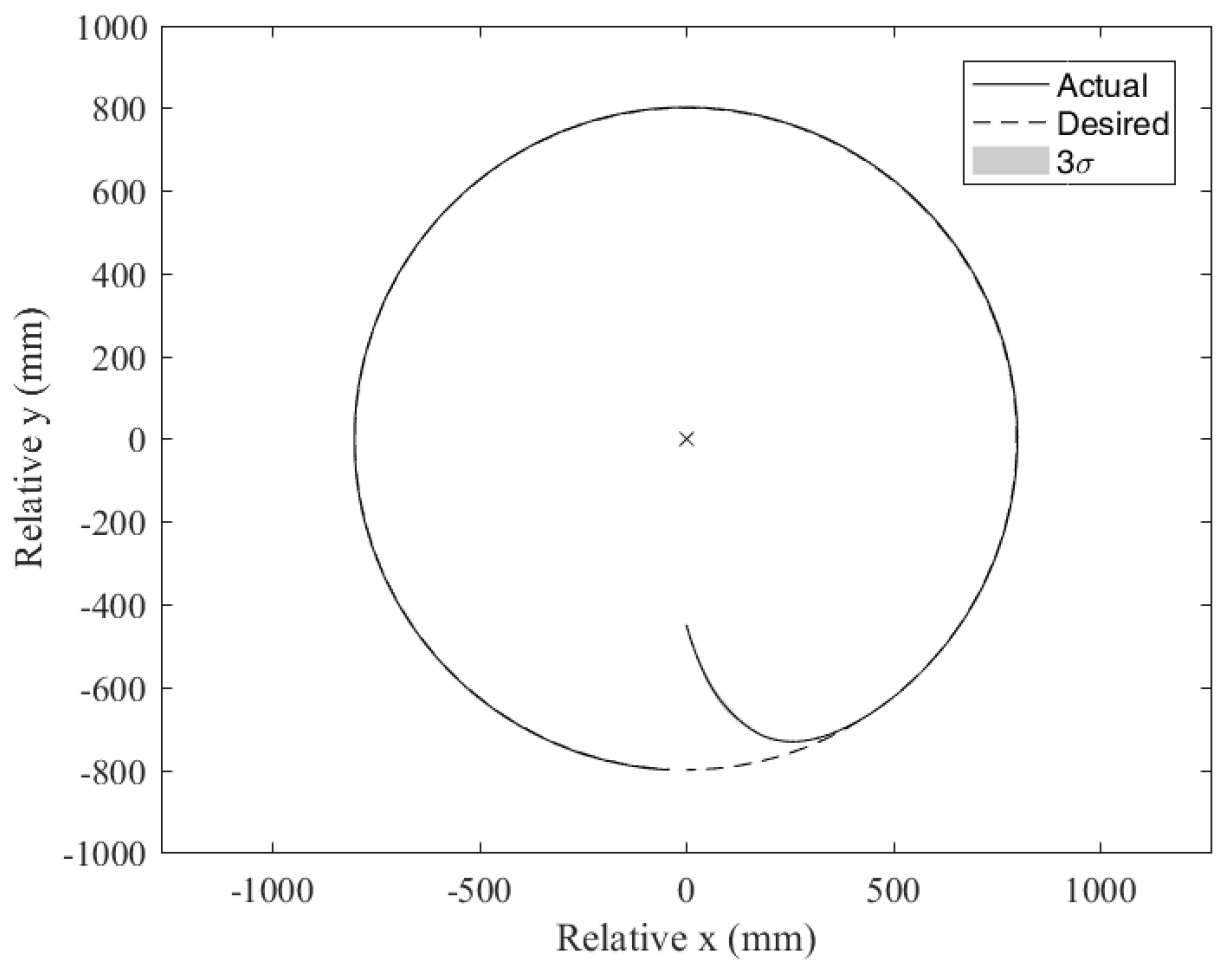

(a) PD control

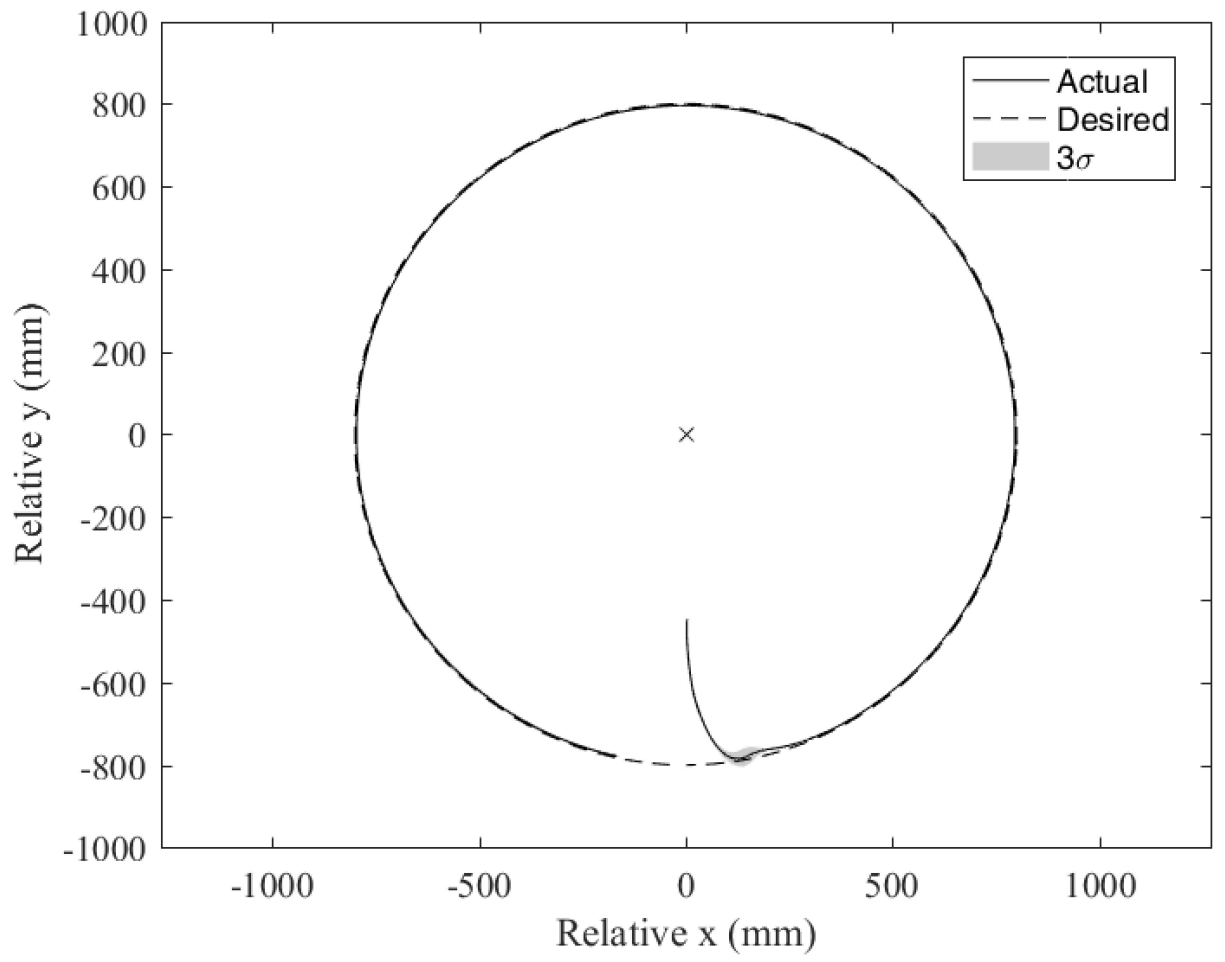

(b) SAC

Figure 5.10: Trajectory tracking experiment with an unknown mass added to the system using (a) PD control and (b) SAC 


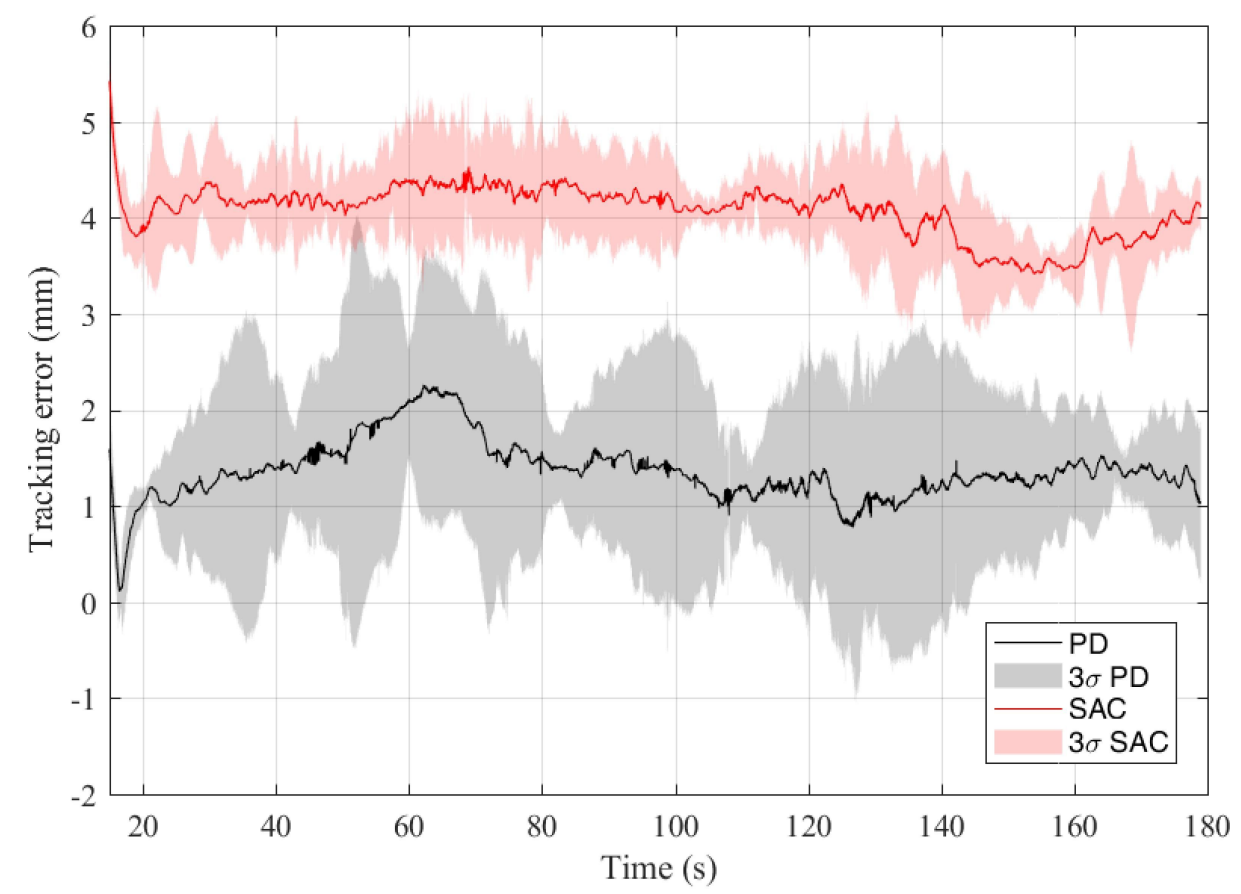

Figure 5.11: Trajectory tracking errors in experiment with an unknown mass added to the system

Figs. 5.10(a) and 5.10(b) represent experimental results with an unknown mass added to the system for PD control and SAC, respectively, and Fig. 5.11 displays the associated tracking errors. Note that the mass was increased from $m_{n o m}=17.24$ $\mathrm{kg}$ to $m_{\text {off }}=26.37 \mathrm{~kg}$ (unknown to the system) representing a substantial increase of approximately 50\%. In application this represents a scenario where the chaser spacecraft has a poor estimate of its mass, for example after capturing debris of uncertain mass, but still has tracking requirements.

Similarly to the results presented for simulations, the addition of an unknown mass to the system had little impact on the match between the desired and actual trajectory in Figs. 5.10(a) and 5.10(b) for PD control and SAC, respectively. Looking more closely at the tracking errors in Fig. 5.11 and comparing them to the nominal case, it is notable that the PD controller performs slightly worse with the addition of an unknown mass to the system, exceeding $2 \mathrm{~mm}$ of tracking error. This is to be expected as the PD controller is tuned for the nominal mass, not the new unknown mass of the system. The tracking error data for PD control is now exhibiting even greater $3 \sigma$ dispersion when compared to SAC than in the previous scenario with 
nominal conditions. It appears that the addition of an unknown mass to the system has caused the PD controller to perform with less precision and repeatability between experiments. In contrast, SAC exhibited virtually no change in performance and the tracking error remained approximately $4 \mathrm{~mm}$ with no increase in data dispersion as the controller adapted to the mass change.

The following experimental scenario investigates the effect of applying perturbation forces to the system without changing its mass. This allows the two effects of perturbation forces and unknown mass changes to be compared in isolation before combining them in the final scenario. 


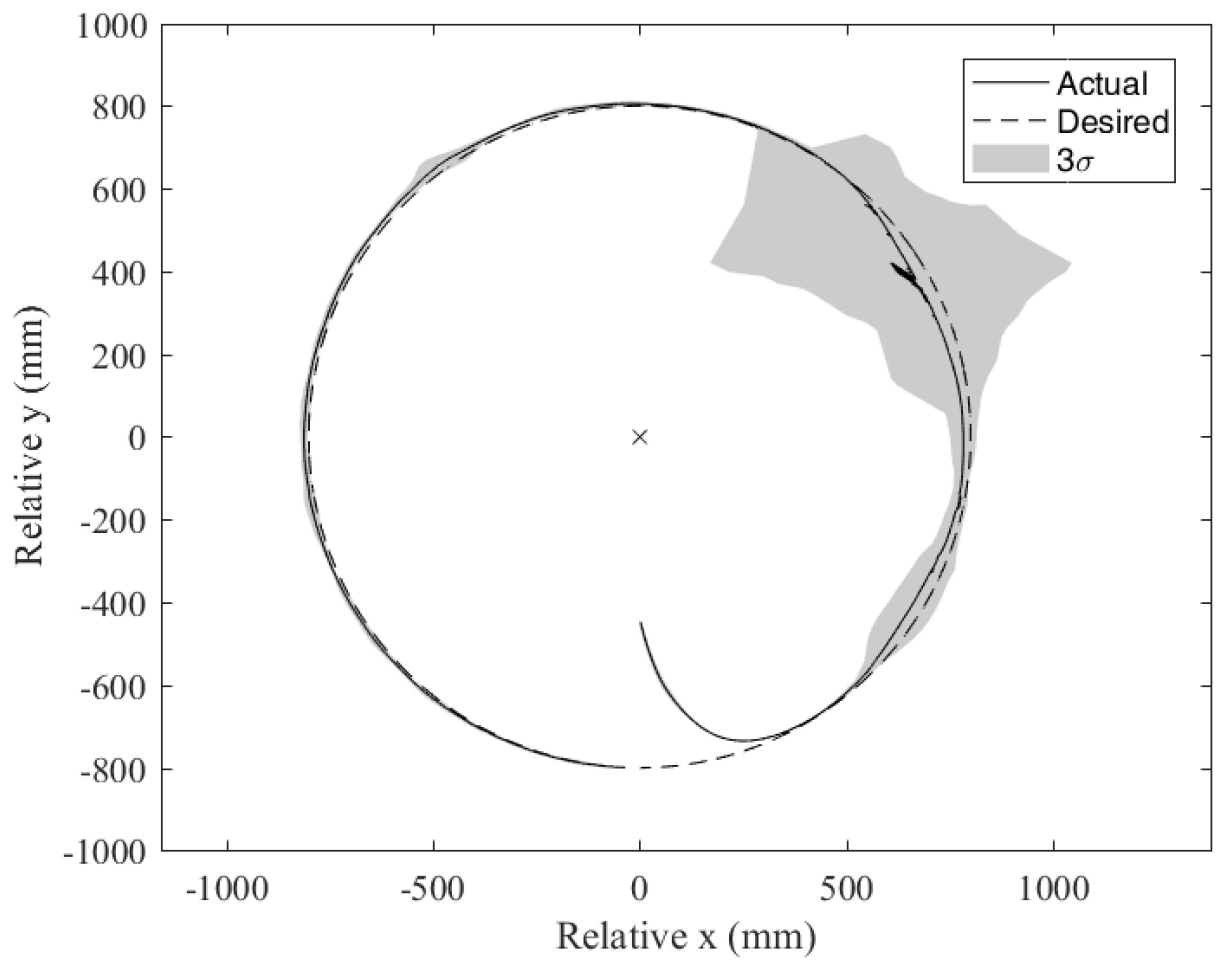

(a) PD control

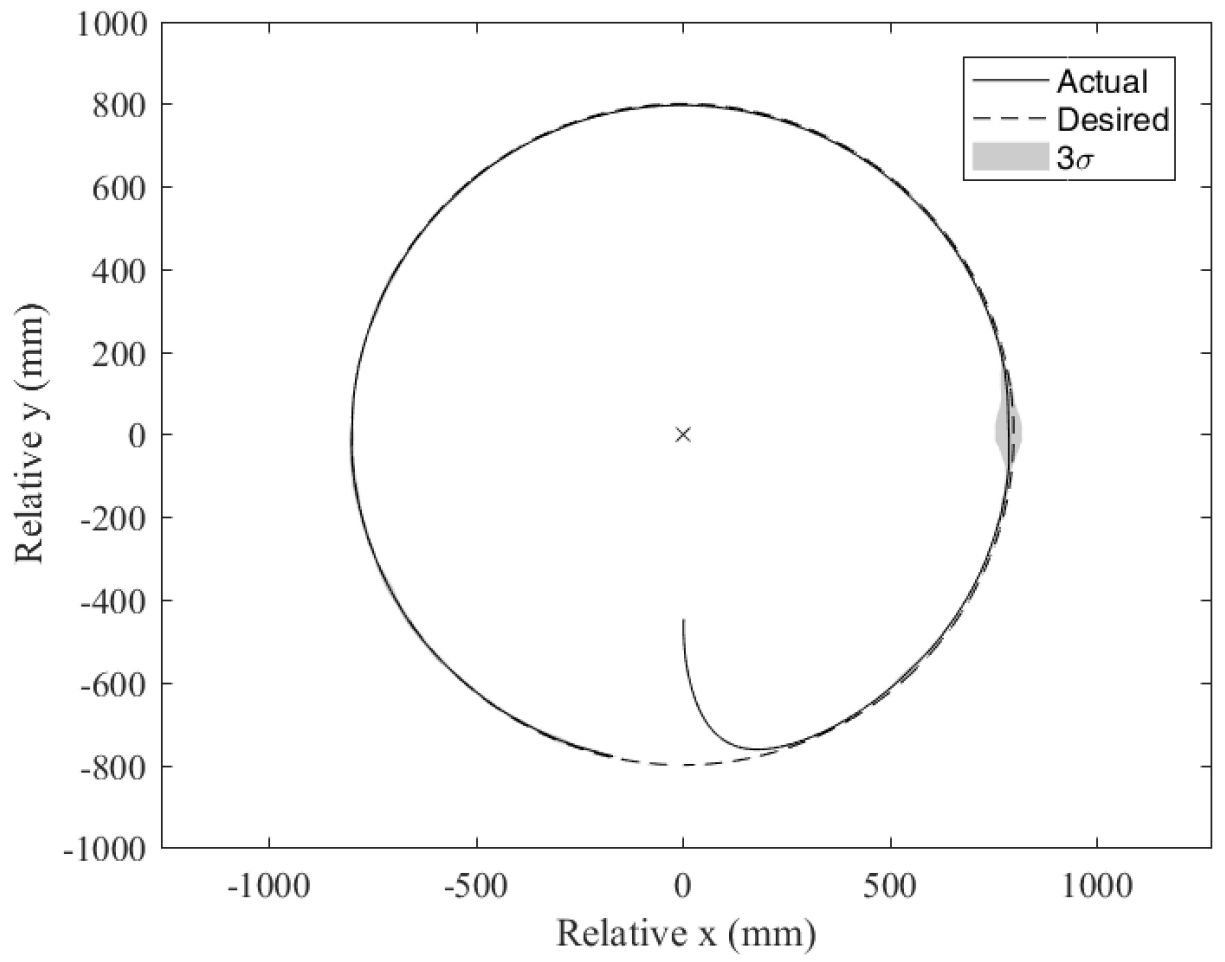

(b) SAC

Figure 5.12: Trajectory tracking experiment with perturbations using (a) PD control and (b) SAC 


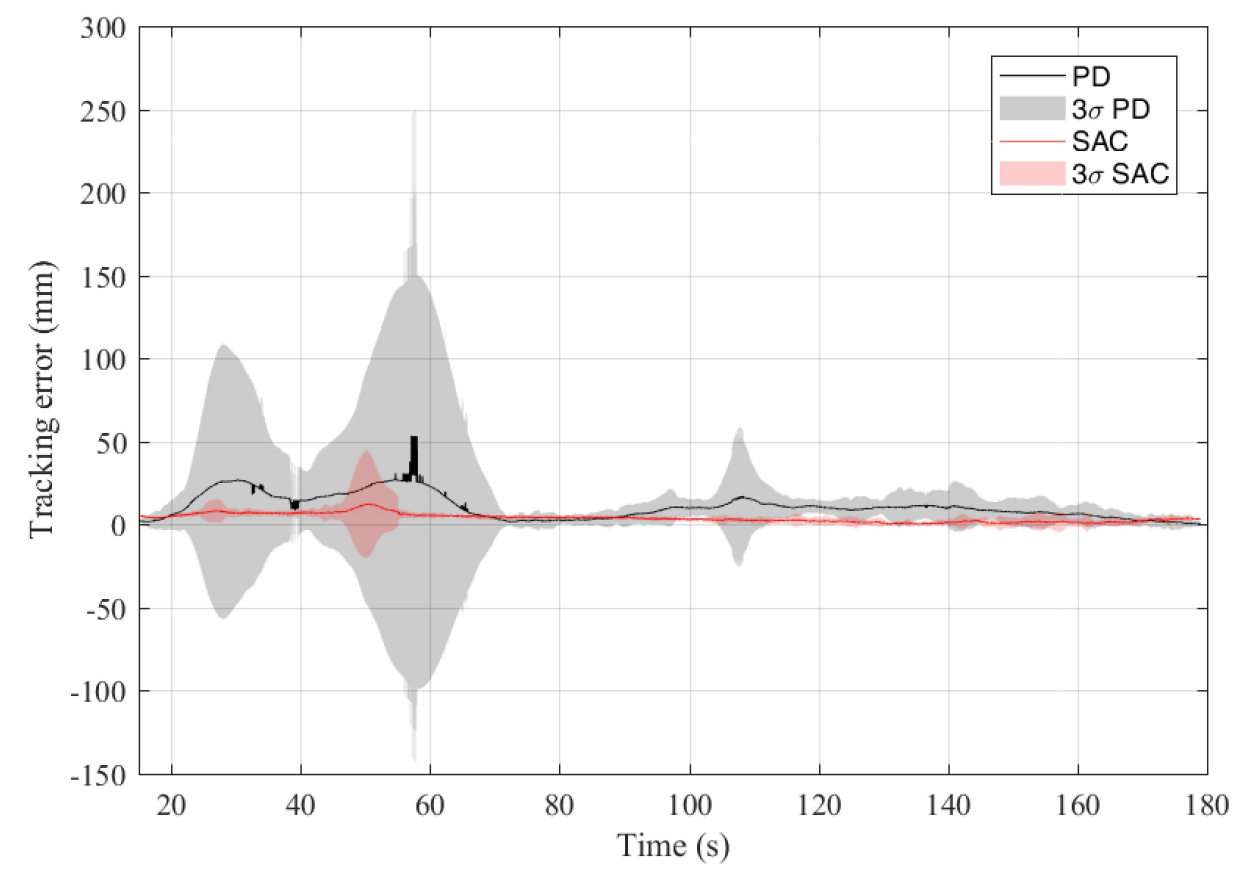

Figure 5.13: Trajectory tracking errors in experiment with perturbations

Figs. 5.12(a) and 5.12(b) represent experimental results with perturbations for PD control and SAC, respectively, and Fig. 5.13 displays the associated tracking errors. Note that the perturbation forces present in experiment are not identical to those in simulation as described in Sec. 5.4 and present a greater challenge to the controller for trajectory tracking than in simulation.

Applying perturbations forces to the system in experiment had a dramatic impact on the match between the desired and actual trajectory for PD control in Fig. 5.12(a) and a much smaller impact for SAC in Fig. 5.12(b), as compared to the nominal and unknown mass change scenarios. Using PD control, the chaser's actual trajectory fluctuates and is visibly different from the desired trajectory over most of its length. The $3 \sigma$ dispersion in tracking error data for $\mathrm{PD}$ control is the most dramatic result. There is little dispersion in the beginning before the fan is turned on, however once the chaser reaches the trajectory and perturbations begin (point B along the trajectory as described in Fig. 5.5) the dispersion begins to grow.

Recall that the fan is located outside the air bearing testbed and is constantly pointed towards the center of the chaser such that the perturbation force is applied in a diagonal direction with negative $x$ and positive $y$ components in the table frame. 
Also recall that the chaser is proceeding along the commanded circular trajectory in a counter-clockwise angular direction and is controlling its attitude such that its cameras are constantly pointing towards the target. During the first quarter of the trajectory, the perturbation force is close to perpendicular with the chaser's velocity (tangential to the circular trajectory) which exacerbates tracking error as it causes a relatively large change in the chaser's direction, steering it off the trajectory as observed. When the chaser has completed one quarter of the circular trajectory and is located directly below the target in the $\mathrm{x}$ direction, the corner of the chaser is pointed at the fan giving it maximum surface area over which to apply air pressure as described in Sec. 5.4. This represents a peak in perturbation forces and it also coincides with the region of worst performance for both controllers. It is the only time SAC tracks poorly enough to produce a visible gap between desired and actual trajectory and a visible $3 \sigma$ dispersion region (see Fig. 5.12(b)). It was observed during experiments that the chaser was struggling to control its position and attitude at this point, regardless of the control strategy, and its air thrusters were firing aggressively to little effect. It is likely that the chaser was experiencing actuator saturation. SAC was able to recover from this quickly and with minimal tracking error due to its adaptive nature but PD control continued to struggle for most of the next quarter of the trajectory, producing a massive $3 \sigma$ dispersion region. This represents poor precision and performance repeatability and a significant collision risk if there were any obstacles located in the dispersion region.

The recovery of PD control from its large $3 \sigma$ dispersion region occurs slightly less than halfway along the trajectory. This recovery was aided by increased distance from the fan, corresponding to weaker perturbation forces, as well as the direction of perturbation forces becoming approximately parallel with the chaser's velocity which minimizes the effect of changing the chaser's direction to steer it off the trajectory.

Another $3 \sigma$ dispersion region occurs for $\mathrm{PD}$ control near point $\mathrm{C}$ along the trajectory as described in Fig. 5.5. The fan speed is increased at this point to compensate for the chaser platform being further away and this is likely the cause of the dispersion region, though it is much smaller than the previous one. SAC has adapted to external conditions well by point $\mathrm{C}$ and does not experience any increase in tracking error 
dispersion. The main result from this experimental scenario is that SAC significantly outperforms PD control in experiment in the presence of perturbation forces.

Now that the effects of unknown mass changes and perturbation forces in experiment have been investigated separately, the final experimental scenario combines these two effects to investigate how SAC and PD control perform under fully off-nominal conditions that are more representative of a real application in debris removal and spacecraft proximity operations. 


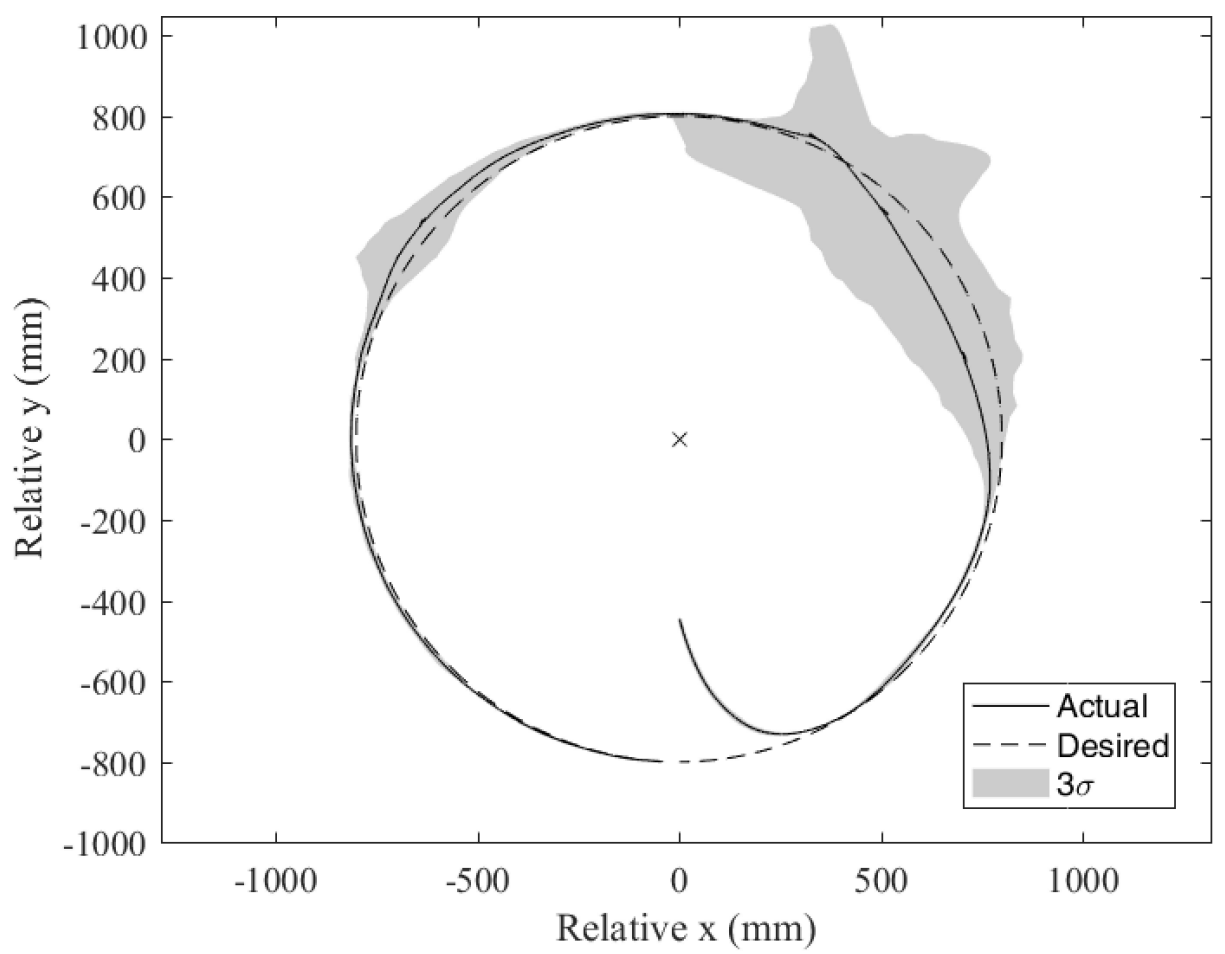

(a) PD control

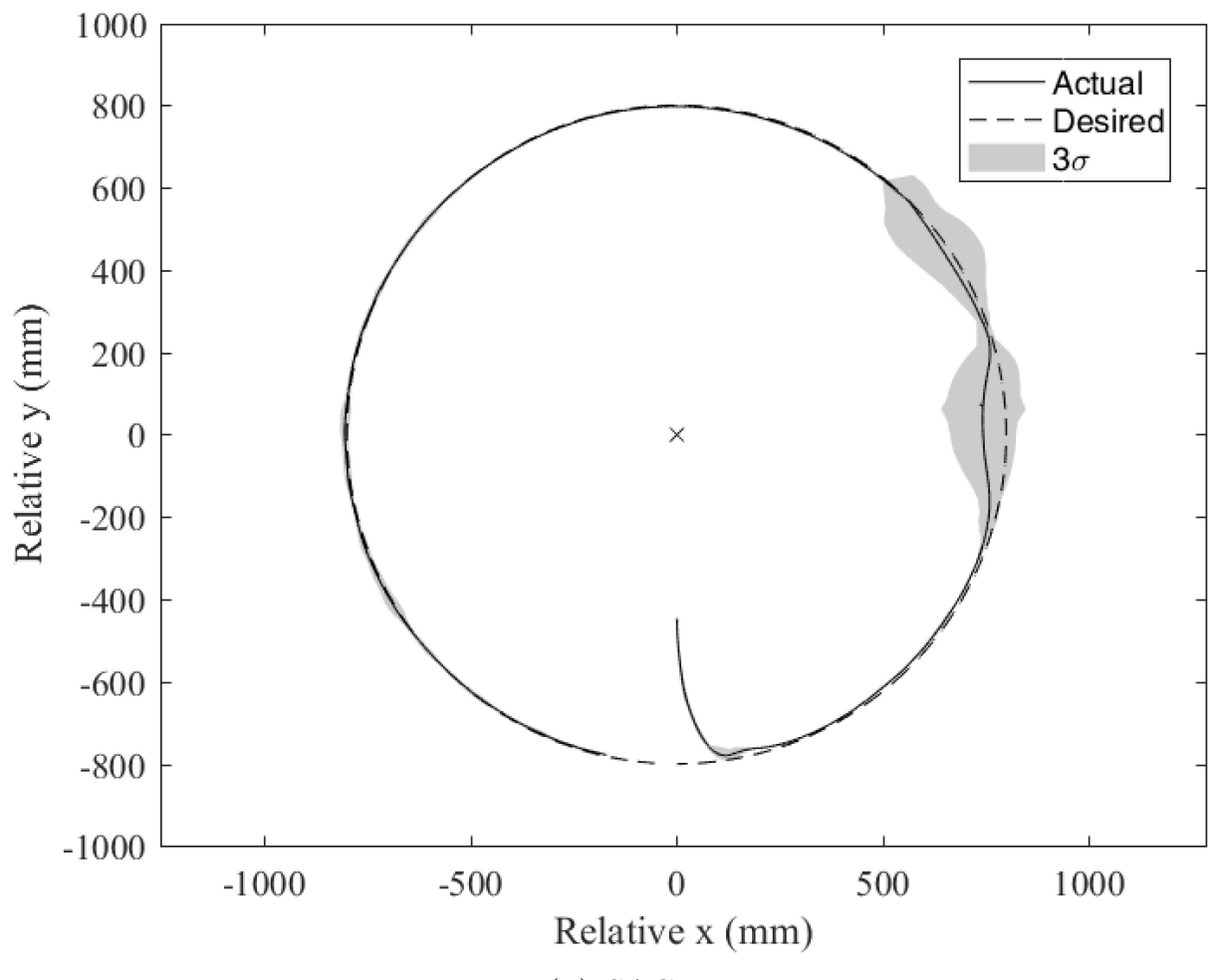

(b) SAC

Figure 5.14: Trajectory tracking experiment under off-nominal conditions using (a) PD control and (b) SAC 


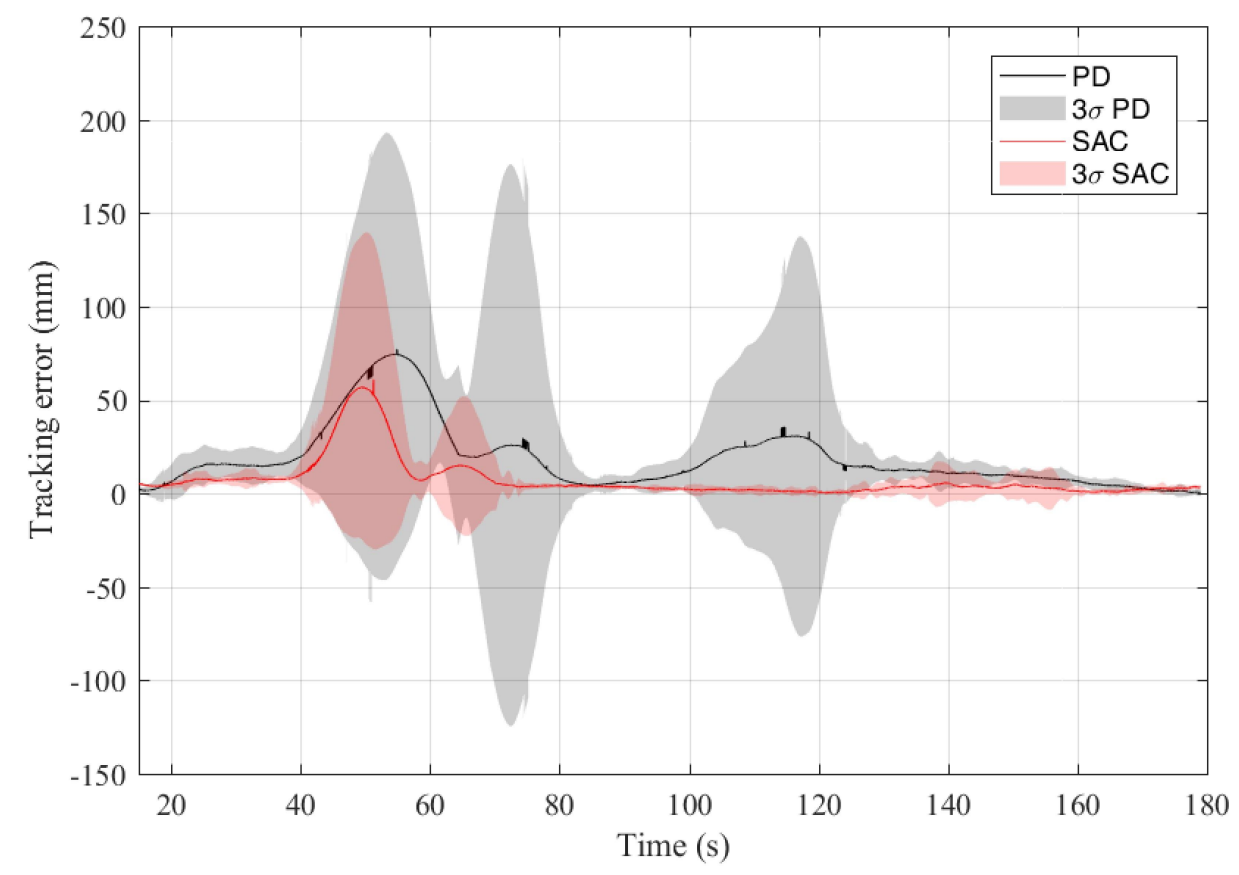

Figure 5.15: Trajectory tracking errors in experiment under off-nominal conditions

Figs. 5.14(a) and 5.14(b) represent experimental results under off-nominal conditions for PD control and SAC, respectively, and Fig. 5.15 displays the associated tracking errors. The chaser had its mass increased by approximately $50 \%$ from $m_{\text {nom }}=17.24$ $\mathrm{kg}$ to $m_{\text {off }}=26.37 \mathrm{~kg}$ without being informed of this change or having its controller gains retuned (to represent capturing space debris of unknown mass) and it is being subjected to perturbation forces as described in Sec. 5.4.

The tracking error profile for both controllers in Fig. 5.15 is similar to Fig. 5.13 where only perturbations were present, though the $3 \sigma$ tracking error dispersion regions have grown larger as a result of the mass increase. This makes sense as PD control was tuned for the nominal mass so an increase in mass would be expected to negatively impact performance.

Comparing the performance of PD control in off-nominal conditions in Fig. 5.14(a) to the performance of PD control with only perturbations in Fig. 5.10(a), it appears that the mass increase delayed the $3 \sigma$ dispersion region. This makes sense as the perturbation force magnitude is approximately equal in both scenarios. An increased mass subjected to the same force would accelerate more slowly. This means that the chaser platform would take longer to deviate from the desired trajectory and longer 
to recover from deviation, as was observed. The largest $3 \sigma$ dispersion region for PD control now ends approximately halfway along the trajectory.

It was observed during experiments that, similarly to the scenario with only perturbations, the chaser was struggling to control its position and attitude after completing approximately one quarter of the trajectory, regardless of the control strategy. Its air thrusters were firing aggressively to little effect and it is likely that the chaser was experiencing actuator saturation. SAC recovered from this more quickly than PD control as before however adaptation took longer as the conditions were more challenging due to the mass increase and SAC experienced a larger $3 \sigma$ dispersion region as a result.

The tracking error for PD control under off-nominal conditions reached a maximum of approximately $24 \mathrm{~cm}$ at three standard deviations from the mean (see Fig. 5.14(a)). To situate this tracking error in context, consider the Astrobee freeflying robots onboard the ISS. Both the Astrobee and the chaser platform in this work are cube shaped with side dimensions of $30 \mathrm{~cm} \mathrm{[4].} \mathrm{This} \mathrm{means} \mathrm{that,} \mathrm{though}$ the risk is low (approximately $0.3 \%$ probability beyond three standard deviations), perturbations and uncertainties could cause the chaser to deviate from its desired trajectory by $80 \%$ of its own body length, representing a significant collision risk in proximity operations as well as making it more difficult to accurately position robotic arms and capture objects.

SAC outperformed PD control in experiment under off-nominal conditions, adapting to the perturbations and mass increase in order to maintain good tracking performance. Fig. 5.16 displays a sample of the time-varying adaptive gains responsible for this superior performance from the stabilizing control gain matrix $\boldsymbol{K}_{e}(t) \in \mathbb{R}^{3 \times 3}$ : 


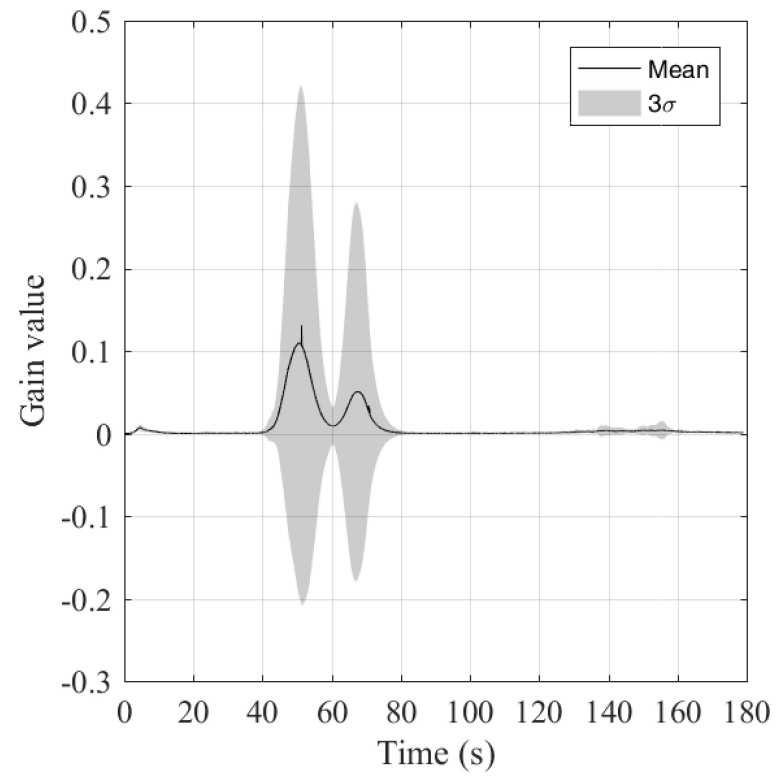

(a) $\mathrm{K}_{e_{11}}$

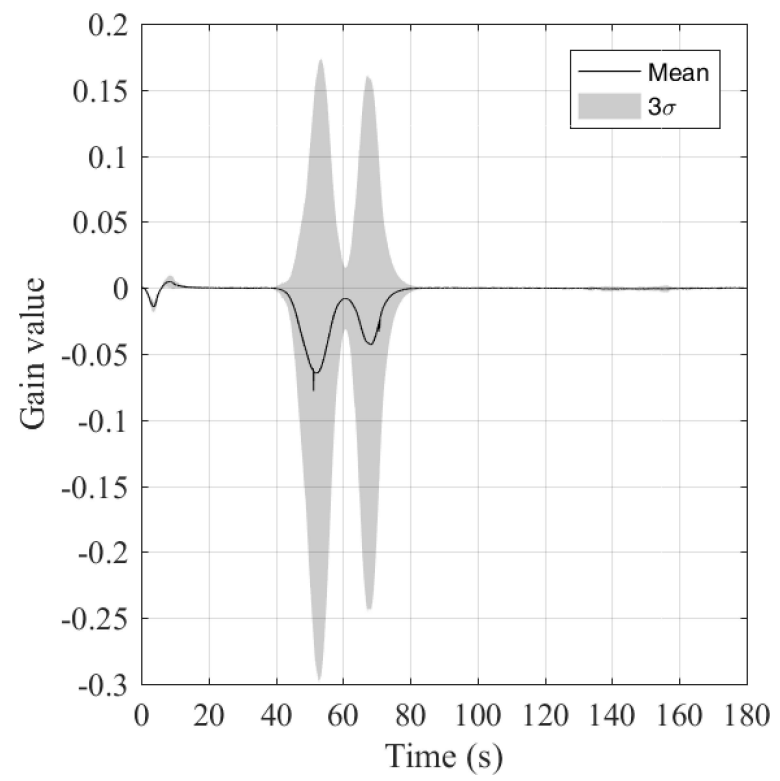

(c) $\mathrm{K}_{e_{21}}$

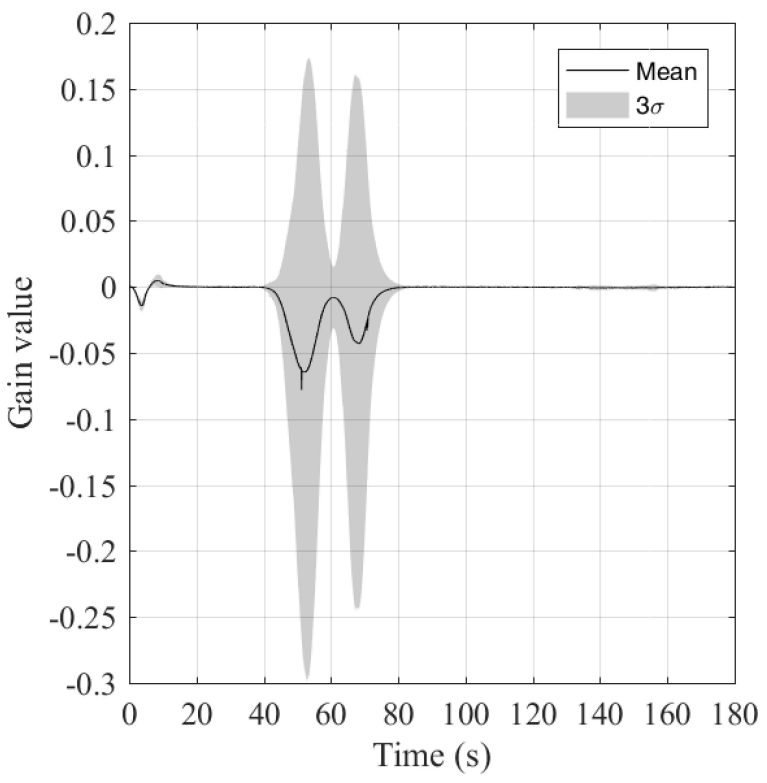

(b) $\mathrm{K}_{e_{12}}$

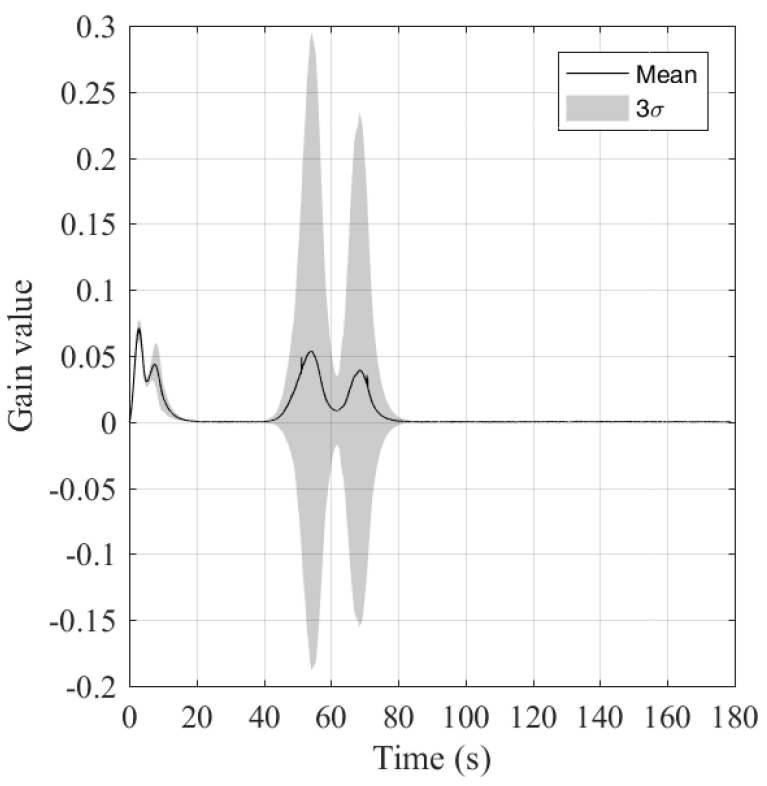

(d) $\mathrm{K}_{e_{22}}$

Figure 5.16: SAC stabilizing control gain matrix $\boldsymbol{K}_{e}(t) \in \mathbb{R}^{3 \times 3}$ in experiment under off-nominal conditions

A portion of the stabilizing control gain matrix is presented as an example of how the gains adapt over time. Note how the gains adapt while reducing the large initial tracking error during the first 15 seconds of the experiment. Though the tracking error is initially large, the $3 \sigma$ dispersion in the gain values between experiments is 
small because the perturbations have not begun yet (recall the perturbation procedure described in Sec. 5.4). This confirms that the adaptive controller behaves in a predictable and precise manner when the experimental conditions are highly consistent.

The adaptive controller experiences its greatest challenge at the point where actuator saturation occurs and SAC struggles during the second quarter of the trajectory as observed in Fig. 5.14(b). Note how the gains vary in response to the tracking error in order to reduce it. The larger $3 \sigma$ dispersion in gain values between experiments during the second quarter of the trajectory is due to random fluctuations in perturbation forces; SAC is adapting to slightly different conditions every time the experiment is repeated. Given that SAC successfully adapts to the worst case scenario it is reasonable to conclude that adaptation also occurs successfully in the less challenging scenarios given that tracking performance is consistently good.

\subsection{Conclusion}

There are three important conclusions from the experimental results, of which the first two agree with simulation conclusions and the third cannot be compared to simulation due to the difference in perturbations:

1. SAC outperformed PD control in all experimental scenarios beyond nominal conditions despite worse tuning of its control gains, further reinforcing the superiority of its performance.

2. Perturbations influence tracking error more significantly than adding an unknown mass to the system, however the presence of both effects has a larger influence than either one in isolation.

3. SAC provides consistent tracking performance in the presence of stochastic perturbations whereas PD control exhibits a relatively large amount of $3 \sigma$ dispersion in tracking error data between experiments. 


\section{Chapter 6}

\section{Conclusion}

This chapter briefly reviews the work motivation, contributions, research and results. The significance of this research to the field of space debris removal and proximity operations is discussed along with possible future work.

\subsection{Thesis Summary}

Orbital debris is a growing problem that poses a collision risk to spacecraft and is expected to worsen exponentially according to Kessler syndrome. Imposing regulations to limit further pollution of orbits with new debris would be helpful but insufficient as it does not address the objects already in orbit; they must be actively removed by a chaser spacecraft which can rendezvous with, capture and tow debris. The technology required for such a spacecraft and proximity operations in general is an important area of research and the motivation behind this work.

Active removal is complicated by the possibility that the chaser spacecraft may have to capture targets of unknown mass and tow them along trajectories while experiencing perturbations. In this case a PID controller cannot guarantee sufficient tracking performance and an adaptive control strategy must be used. Linear continuous control laws require knowledge of the debris and perturbations that may not be available and indirect adaptive control is computationally expensive in its efforts to determine debris parameters. Direct adaptive control does not require knowledge of the debris and has been successfully implemented before in a similar experiment by Ulrich et al. [11] using Simple Adaptive Control (SAC).

The author made significant contributions to designing and building the facility necessary to perform simulations and experiments for this work, the Spacecraft Proximity Operations Testbed (SPOT) located in the Spacecraft Robotics and Control Laboratory at Carleton University. Specifically the author was responsible for 
creating the software environment that enabled simulations and experiments to be performed.

The SPOT facility was used to simulate a chaser spacecraft tracking a circular inspection trajectory around target debris. This represents a typical trajectory that could be performed prior to a rendezvous and capture maneuver. Four different sets of conditions were tested with the worst case scenario involving the chaser spacecraft having an unknown mass in the presence of perturbation forces. There were two important conclusions from the simulations. The first is that SAC outperformed PD control in all scenarios beyond nominal conditions despite worse tuning of its control gains. The second is that perturbations influence tracking error more significantly than adding an unknown mass to the system, however the presence of both effects has a larger influence than either one in isolation.

The scenarios tested in simulation were experimentally validated using the SPOT facility. The only difference between simulation and experiments is that the perturbation forces in simulation were provided by a simple sinusoidal function whereas in experiment they were provided by a fan blowing air that the sinusoidal function did not accurately model. There were three important conclusions from the experiments. The first and second conclusions are identical to those from simulations. The third is that SAC provides consistent tracking performance in the presence of stochastic perturbations whereas PD control exhibits a relatively large amount of dispersion in tracking error data between experiments.

\subsection{Future Work}

Future work investigating the suitability of SAC for trajectory tracking during debris removal and proximity operations could include a parametric study on the influence of varying magnitudes of perturbation forces on tracking performance. The perturbation forces would need to be measured accurately for this kind of study and varied over a specific range that goes beyond the worst case forces expected for a particular application in proximity operations.

Future work could also focus on more realistic and comprehensive experiments involving additional subsystems that would be present in a real mission. For example, 
the Spacecraft Robotics and Control Laboratory at Carleton University is currently developing machine vision and robotic arms for use on the chaser platform. An experiment where SAC performed trajectory tracking while the target debris was surveyed using machine vision or captured using a robotic arm would be a more realistic test of greater relevance to real debris removal missions, which must take place in order to prevent orbits from becoming too dangerous to use.

Finally, an exciting possibility for future work could be to test SAC for proximity operations onboard the ISS through the experimental facilities provided by Astrobee. 


\section{Bibliography}

[1] Shan, M., Guo, J., and Gill, E., "Review and Comparison of Active Space Debris Capturing and Removal Methods," Progress in Aerospace Sciences, Vol. 80, 2016, pp. 18-32.

[2] Kessler, D. J. and Cour-Palais, B. G., "Collision Frequency of Artificial Satellites - The Creation of a Debris Belt," Journal of Geophysical Research, Vol. 83, No. A6, 1978, pp. 2637-2646.

[3] Wormnes, K., Le Letty, R., Summerer, L., Krag, H., Schonenborg, R., DuboisMatra, O., Luraschi, E., Delaval, J., and Cropp, A., "ESA Technologies for Space Debris Remediation," 6th European Conference on Space Debris, Darmstadt, Germany, 2013.

[4] Smith, T., Barlow, J., Bualat, M., Fong, T., Provencher, C., Sánchez, H. J., and Smith, E. P., "Astrobee: a New Platform for Free-flying Robotics on the International Space Station," 13th International Symposium on Artificial Intelligence, Robotics, and Automation in Space (i-SAIRAS), 2016.

[5] Clohessy, W. H., "Terminal Guidance System for Satellite Rendezvous," Journal of the Aerospace Sciences, Vol. 27, No. 9, 1960, pp. 653-658.

[6] Fehse, W., Automated Rendezvous and Docking of Spacecraft, Cambridge University Press, Cambridge, United Kingdom, 2003.

[7] Ogata, K., Modern Control Engineering, Vol. 5, Prentice Hall, Upper Saddle River, USA, 2010.

[8] Egardt, B., Stability of Adaptive Controllers, Springler-Verlag, Berlin, Germany, 1979 .

[9] de Querioz, M. S., Vikram, K., and Qiguo, Y., "Adaptive Nonlinear Control of Multiple Spacecraft Formation Flying," Journal of Guidance, Control, and Dynamics, Vol. 23, No. 3, 2000, pp. 385-390.

[10] Barkana, I., "Simple adaptive control-a stable direct model reference adaptive control methodology-brief survey," International Journal of Adaptive Control and Signal Processing, Vol. 28, No. 7-8, 2014, pp. 567-603.

[11] Ulrich, S., Saenz-Otero, A., and Barkana, I., "Passivity-Based Adaptive Control of Robotic Spacecraft for Proximity Operations Under Uncertainties," Journal of Guidance, Control, and Dynamics, Vol. 39, No. 6, 2016, pp. 1441-1450. 
[12] de Ruiter, A., Damaren, C., and Forbes, J., Spacecraft Dynamics and Control, John Wiley \& Sons, West Sussex, UK, 2013.

[13] Broussard, J. and O'Brien, M., "Feedforward control to track the output of a forced model," IEEE Transactions on Automatic Control, Vol. 25, No. 4, Aug 1980, pp. 851-853.

[14] Narendra, K. S., Tripathi, S. S., Luders, G., and Kudva, P., Adaptive Control Using Lyapunov's Direct Method, Yale University, New Haven, CT, 1971.

[15] Narendra, K. S. and Annaswamy, A. M., Stable Adaptive Systems, SpringerVerlag, Englewood Cliffs, NJ, 1989.

[16] Ioannou, P. A. and Kokotovic, P., Adaptive Systems with Reduced Models, Springer-Verlag, New York, 1983.

[17] Ioannou, P. and Kokotovic, P., "Instability analysis and improvement of robustness of adaptive control," Automatica, Vol. 20, No. 5, 1984, pp. 583 - 594.

[18] Barkana, I. and Kaufman, H., "Direct adaptive control with bounded tracking errors," The 22nd IEEE Conference on Decision and Control, 1983, pp. 221-222.

[19] Barkana, I. and Kaufman, H., "Robust simplified adaptive control for a class of multivariable systems," The 24th IEEE Conference on Decision and Control, 1985, pp. 141-146.

[20] Fradkov, A. L., "Quadratic Lyapunov functions in the adaptive stability problem of a linear dynamic target," Siberian Mathematical Journal, Vol. 17, No. 2, Mar 1976, pp. 341-348. 